\title{
Modelos de contaminação sequencial em alguns sistemas de partículas
}

\section{Marco Antồnio Giacomelli}

Tese apresentada ao Instituto de Matemática e Estatística da Universidade de São Paulo para a obtenção do título de Doutor em Estatística

Área de concentração: Probabilidade

Orientador: Prof. Dr. Serguei Popov 


\section{Modelos de Contaminação Sequencial em Alguns Sistemas de Partículas}

Este exemplar corresponde à redação final da tese devidamente corrigida e defendida por Marco Antônio Giacomelli e aprovada pela comissão julgadora.

Banca examinadora:

- Prof. Dr. Serguei Popov /IME-USP (Presidente)

- Prof. Dr. Pablo Augusto Ferrari /IME-USP

- Profa. Dra. Marina Vachkovskaia /IMECC-UNICAMP.

- Prof. Dr. Oswaldo Scarpa Magalhães Alves / UFG

- Prof. Dr. Márcio Argollo Ferreira de Menezes /UFF-RJ 
Dedicatória:

A meus pais e meu afilhado Cristiano 
Agradecimentos:

- em especial ao Prof. Serguei pela motivação, determinação, amizade e ótima orientação, sem a qual eu não teria chegado à conclusão deste trabalho.

- aos colegas William de Souza Pereira, José Domingo Restrepo Alvarez e José Elmo de Menezes pela amizade e companheirismo.

- ao Departamento de Estatística da Universidade Federal do Rio Grande do Sul por conceder afastamento para realizar o curso. 


\section{Sumário}

$\begin{array}{ll}\text { Resumo } & 3\end{array}$

$\begin{array}{ll}\text { Abstract } & 4\end{array}$

Lista de abreviaturas e notações $\quad 5$

$\begin{array}{ll}\text { Introdução } & 7\end{array}$

1 Modelo com mesmas taxas de salto em todos sítios 10

1.1 Definições . . . . . . . . . . . . . . . . . . . . . . 10

1.2 O algoritmo para a simulação . . . . . . . . . . . . . . 17

1.3 Programa em Scilab . . . . . . . . . . . . . . . . . . . . . . . . 19

1.4 Alguns exemplos da execução do programa . . . . . . . . . . . . . . . . 24

1.5 Sobre a medida invariante do modelo centrado . . . . . . . . . . . . . . 31

1.6 Uma medida dominante para o modelo centrado . . . . . . . . . . . 43

1.7 Conexão com o Movimento Browniano . . . . . . . . . . . . . . . . . . 46

1.8 Conclusões . . . . . . . . . . . . . . . . . . . . . . . . . . . 51

2 Propagação de infecção entre Movimentos Brownianos independentes 52

2.1 Definindo o modelo . . . . . . . . . . . . . . . . . . . . . . 52

2.2 Passeio Aleatório Simples como uma aproximação discreta ao Movimento Browniano . . . . . . . . . . . . . . . . 5 56 
2.3 Simulando o Movimento Browniano . . . . . . . . . . . . . . 58

$2.4 \mathrm{O}$ algoritmo para a simulação . . . . . . . . . . . . . . . 60

2.5 Programa em Scilab . . . . . . . . . . . . . . . 61

2.6 Alguns exemplos da execução do programa . . . . . . . . . . . . . . . 64

2.7 Programa com opção de gráfico . . . . . . . . . . . . . . . 67

2.8 Conclusões . . . . . . . . . . . . . . . . . . . . . . . . 70

3 Modelo com partículas ativas nos sítios contaminados e inativas nos $\begin{array}{ll}\text { normais } & 72\end{array}$

3.1 Definindo o modelo . . . . . . . . . . . . . . . . . 72

3.2 O Teorema Ergódico Subaditivo . . . . . . . . . . . . . . . . . 74

3.3 Aplicando o Teorema Ergódico Subaditivo . . . . . . . . . . . . . 74

3.4 Obtendo uma cota inferior para a velocidade de contaminação . . . . . 85

3.5 Conclusões . . . . . . . . . . . . . . . . . . . . . . . . . . . . . 90

$\begin{array}{ll}\text { Referências Bibliográficas } & 91\end{array}$ 


\section{Resumo}

Estudamos a propagação de infecção em alguns sistemas de partículas em $\mathbb{Z}$ e também em $\mathbb{R}$. Convencionamos que no instante inicial sítios não positivos deverão conter partículas contaminadas, enquanto os positivos conterão partículas normais. Dessa forma, o sítio zero será a fronteira inicial entre contaminados e normais. Sempre que uma partícula contaminada alcançar um sítio normal, este ficará contaminado. Para avaliar a evolução da infecção definimos a velocidade de contaminação do processo como $v:=\lim _{t \rightarrow \infty} \frac{Z_{t}}{t}$, sendo $Z_{t}$ a fronteira atual. No primeiro modelo, a cada partícula associamos um relógio aleatório que soa de acordo com um Processo de Poisson de taxa 1. Este relógio é referente aos instantes de saltos da partícula. Já, no segundo modelo, tratamos da contaminação em Movimentos Brownianos independentes, os quais aproximamos por Passeios Aleatórios Simples, cuja distância entre dois vizinhos mais próximos é $\Delta \in(0,1)$. No terceiro processo, partículas infectadas se deslocam à direita segundo um processo de Poisson de taxa 1 , enquanto as normais permanecem paradas (taxa zero). Quando uma partícula infectada atinge um sítio normal, as partículas acordam e passam a se deslocar com taxa 1 . Simulamos os modelos dos Capítulos 1 e 2 num intervalo finito até um tempo $T$ pré-fixado, observando a velocidade estimada e também algumas estatísticas. No terceiro Capítulo obtemos alguns resultados teóricos para o modelo. 


\section{Abstract}

We study the infection spreading in some particle system models, with state space $\mathbb{Z}$ and $\mathbb{R}$. Here we assume that nonpositive sites contain infected particles, while the positive sites are healthy. Thus, zero is the initial boundary between infected and healthy particles. Whenever an infected particle reach a healthy site, all particles there become infected. The goal is to analyse the evolution of infection spreading, for which we set $v:=\lim _{t \rightarrow \infty} \frac{Z_{t}}{t}, Z_{t}$ being the actual boundary. In the first model, to each particle in site $x \in \mathbb{Z}$ we attach a random clock that rings according to a Poisson Process of intensity 1 . That clock relates to the moments of particle jumps. The second model is about the infection spreading between independent Brownian Motions, and we approach it through Simple Random Walks with distances between two nearest neighbours being $\Delta \in(0,1)$. In the third process, infected particles displace to the right with intensity 1 , while the healthy ones are sleeping (intensity zero). When an infected particle reaches a healthy site, particles are waked up and starts to jump with intensity 1 . We simulate the models of chapters 1 and 2 in a finite interval until an arbitrary time $T$, analysing the estimated speed, and furthermore some other statistics. In the third Chapter, we get some rigours results for the model. 


\section{Lista de abreviaturas e notações}

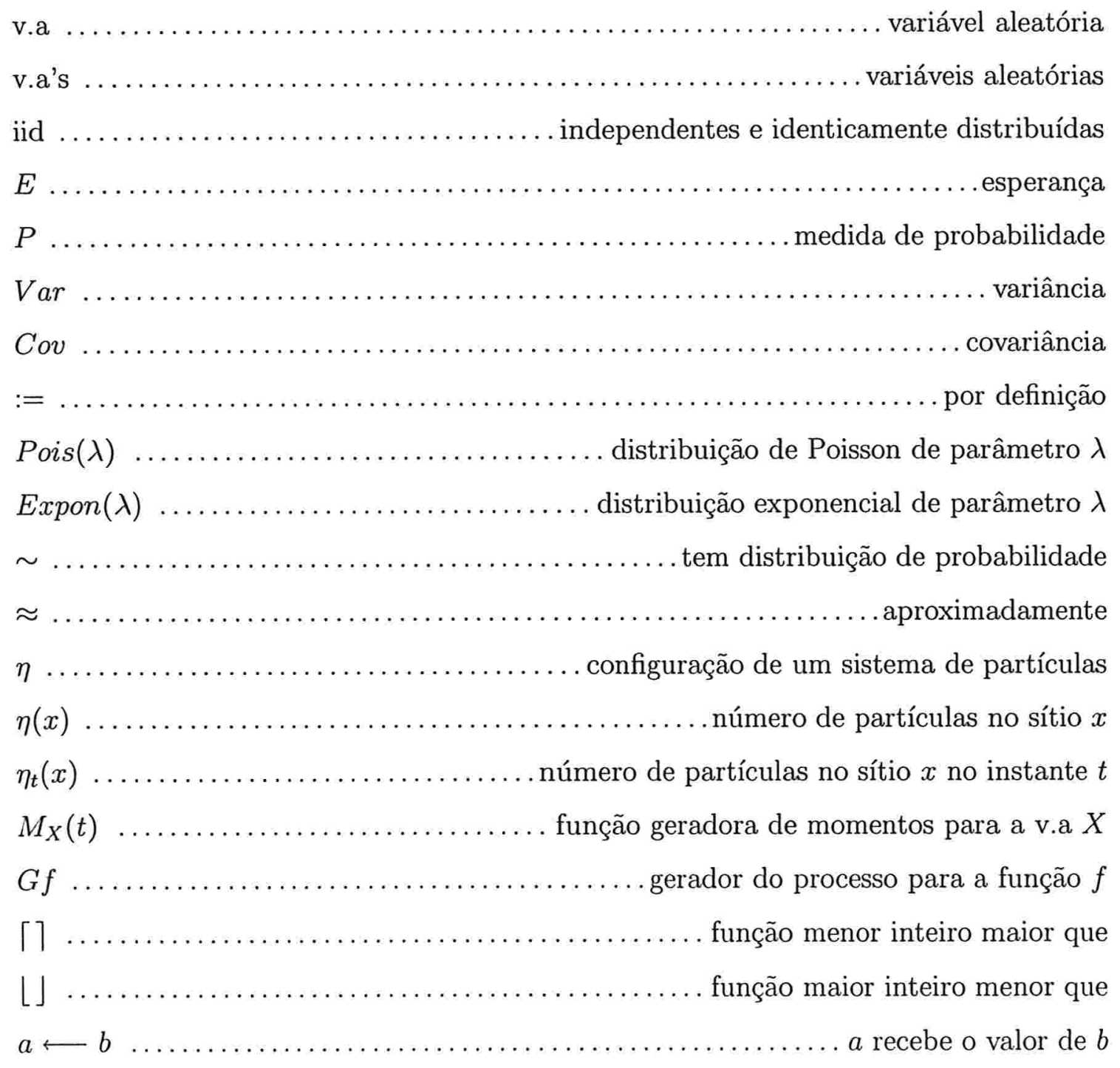


sup supremo de um conjunto

inf . ínfimo de um conjunto

$I_{\{A\}}$ função indicadora do evento $A$

$\mathrm{o}(h)$ $. \lim _{h \downarrow 0} \frac{o(h)}{h}=0$

$O(h)$ $\left|\frac{O(h)}{h}\right| \leq c, c>0$ 


\section{Introdução}

Sistemas de partículas são processos Markovianos para descrever a evolução no tempo e no espaço de infinitas componentes interagentes. Podemos pensar que estas partículas são unidades que compõem um sistema, como por exemplo: um meio físico, biológico ou econômico. O espaço em que estas partículas evoluem pode ser desde o conjunto dos números naturais até espaços mais complexos. Em relação ao tempo pode ser discreto ou contínuo. Nestes sistemas existe a componente aleatória que pode estar na configuração inicial (quantidade de partículas no instante inicial) ou durante o decorrer da evolução do processo. A maneira como estas partículas interagem entre si dá origem a diferentes modelos já amplamente estudados: Modelo do Votante, Processo de Contato, Processo de Exclusão e Percolação de Primeira Passagem. Nos modelos de Percolação de Primeira Passagem, estuda-se o avanço, por exemplo, de uma epidemia sobre um espaço, que pode ser o conjunto $\mathbb{Z}^{d}, d \geq 1$. O contágio se dá quando um sítio sadio tem contato com outro vizinho adjacente, que já estava contaminado. Pode-se ainda introduzir um parâmetro $p$ tal que o sítio ficará contaminado e imune com probabilidades $p$ e $1-p$, respectivamente. Outra possibilidade é introduzir uma variável aleatória representando o tempo de vida de uma partícula no sistema. Há ainda modelos em que novas partículas vão sendo introduzidas no sistema com o passar do tempo. Este é o caso, por exemplo, do Passeio Aleatório com Ramificação ([21]).

Um dos primórdios da teoria de sistemas de partículas interagentes aparece nos tra- 
balhos de Ehrenfest (1907), sugerindo modelos de ocupação de urnas com um número finito de bolas. Também, pode-se observar relações com a Mecânica Estatística, onde citamos Boltzmann (1902) e Gibbs (1902). Posteriormente, Harris (1965) introduziu o modelo Processo de Exclusão. Também salientamos as contribuições de Spitzer (1970) e Liggett (1977) para estes processos. Um histórico mais detalhado encontra-se em [8].

Aplicações de sistemas de partículas estão em diversas áreas, e a interpretação será de acordo com a área de aplicação. Por exemplo, o fluxo das partículas pode ser interpretado como o avanço de uma epidemia, como a disseminação de uma informação, ou ainda como uma reação em cadeia em um sistema físico-químico.

No Capítulo 1 estudamos o modelo em que partículas se deslocam de acordo com um Processo de Poisson Homogêneo de taxa 1, independentemente, em $\mathbb{Z}$. Estabelecemos que no instante inicial sítios negativos (incluindo o zero) estarão contaminados, enquanto os positivos serão normais. À medida que uma partícula contaminada se deslocar para o sítio vizinho à direita, então irá contaminando os sítios normais. Propomos obter uma aproximação para a velocidade de contaminação $\lim _{t \rightarrow \infty} \frac{Z_{t}}{t}, Z_{t}$ sendo a fronteira entre contaminados e normais no instante $t$. Mostramos que a medidaproduto de Poisson não é a medida invariante para o modelo centrado, o qual definiremos oportunamente. Contudo, através de simulações, observamos que a velocidade de contaminação estimada fica próxima da velocidade teórica obtida com a suposição de que a medida-produto fosse a invariante. Também mostramos que para frente da infecção (para sítios normais que estão à direita de $Z_{t}$ ) a medida-produto de Poisson é dominante para o processo. Para finalizar o Capítulo 1 estabelecemos uma conexão com o Movimento Browniano padrão, ou seja, admitindo que a distância entre dois vizinhos mais próximos é $\lambda \in(0,1)$ e que cada partícula se desloca à direita segundo um Processo de Poisson de taxa $\lambda^{-2}$, verificamos que quando $\lambda \downarrow 0$ a trajetória da partícula converge para o Movimento Browniano padrão.

Para o Capítulo 2 o modelo teórico consiste em partículas realizando um Movimento 
Browniano padrão, independente um do outro. O objetivo é estudar a velocidade de infecção. Para tal fim utilizamos simulações em uma caixa finita (um intervalo finito em $\mathbb{R})$, a qual cada sítio tem uma distância $\Delta \in(0,1)$ em relação ao seu vizinho mais próximo. Cada partícula em um sítio tem probabilidade $\frac{1}{2}$ de se deslocar para seu vizinho mais próximo. Através de simulações conseguimos observar que a velocidade de contaminação é $\frac{1}{2}$, estando em concordância com o resultado empírico obtido no Capítulo 1.

O terceiro Capítulo foi dedicado ao modelo em que partículas sadias ficam inativas até serem "acordadas" por uma contaminada. As partículas contaminadas se deslocam de acordo com um Processo de Poisson Homogêneo de taxa 1. Quando uma partícula sadia é acordada, então passa a ter taxa de deslocamento igual a 1. Este último modelo é semelhante ao "Frog Model" (ver [1], [2] e [19]), que começa com apenas uma partícula contaminada na origem. O principal resultado deste capítulo é a existência da velocidade e o fato de que $v>1$. O teorema principal para obter esta cota inferior foi o Teorema Ergódico Subaditivo, devido a Kingman ([15]).

Nas simulações dos modelos utilizamos a linguagem de programação Scilab, do Instituto INRIA, que é similar ao Matlab da Mathworks. Uma referência para o Scilab está em [9]. 


\section{Capítulo 1}

\section{Modelo com mesmas taxas de salto em todos sítios}

\subsection{Definições}

Considere que o conjunto dos números inteiros corresponde a sítios onde serão alocadas, de maneira independente, uma quantidade aleatória de partículas. Essa quantidade seguirá uma determinada distribuição de probabilidade. Vamos assumir que no tempo zero, também denominado de instante inicial, em cada sítio seja atribuída uma quantidade de partículas segundo uma distribuição de Poisson de parâmetro $\lambda$. Sendo assim, essas variáveis aleatórias são mutuamente independentes e identicamente distribuídas, ou seja, a distribuição conjunta do número de partículas nos sítios é o produto dessas distribuições de Poisson, que denominaremos de medida-produto. $\mathrm{O}$ número de partículas em um sítio $x$, no instante atual $t$, é denotado por $\eta_{t}(x)$. Para o instante inicial, $\eta_{0}(x)$ será a configuração inicial do sítio $x$. Quando usarmos $\eta_{t}$ estaremos nos referindo à configuração global do modelo.

Também assuma que no instante inicial partículas nos sítios negativos, e no zero, estão contaminadas e nos positivos estão normais. Além disso, quando uma partícula 
contaminada atingir um sítio normal, todas lá contidas imediatamente ficarão contaminadas. Assumiremos que partículas dentro de um mesmo sítio não interagem entre si, assim como aquelas de sítios diferentes. O sítio zero é dito fronteira inicial entre as partículas contaminadas e as normais.

A dinâmica do modelo é tal que partículas se deslocam da esquerda à direita. Os instantes entre estes deslocamentos (saltos) de uma partícula ocorrem de acordo com um Processo de Poisson Homogêneo de taxa 1. A este Processo de Poisson chamaremos de relógio da partícula. Desta forma, para cada partícula estará associado um relógio, e estes são mutuamente independentes. Após o instante inicial, em um sítio $x$, o relógio associado à $k$-ésima partícula irá gerar uma quantidade $\tau_{k}^{(x)} \sim \operatorname{Expon}(1)$, $k=1, \ldots, \eta_{t}(x)$. O instante da primeira partícula em $x$, que vai saltar à direita, será

$$
\tau^{(x)}:=\min \left\{\tau_{k}^{(x)}: k=1, \ldots, \eta_{t}(x)\right\}
$$

Como $\left\{\tau_{k}^{(x)}: k=1, \ldots, \eta_{t}(x)\right\}$ são iid, então $\tau^{(x)} \sim \operatorname{Expon}\left(\eta_{t}(x)\right)$. Este mesmo mecanismo também ocorrerá, de maneira independente, nos outros sítios. É através desta dinâmica que o modelo irá evoluir no tempo.

Formalmente, a relação entre as taxas de transição $\eta_{t}(x)$ e o processo é dada pelo gerador infinitesimal, a seguir enunciado.

Definição 1.1.1. O gerador infinitesimal do processo é definido como

$$
G f\left(\eta_{t}\right):=\sum_{x \in \mathbb{Z}} \eta_{t}(x)\left(f\left(\eta_{t}^{x, x+1}\right)-f\left(\eta_{t}\right)\right)
$$

onde a configuração $\eta_{t}^{x, x+1}$ é tal que

$$
\eta_{t}^{x, x+1}(z)= \begin{cases}\eta_{t}(z), & \text { se } z \neq\{x, x+1\} \\ \eta_{t}(z)-1, & \text { se } z=x \\ \eta_{t}(z)+1, & \text { se } z=x+1\end{cases}
$$


Interpretando $\eta_{t}^{x, x+1}$, significa que o sítio da posição $x$ perde uma partícula e esta se desloca para o próximo da direita. Com relação à função $f, f: \mathbb{N}^{\mathbb{Z}} \longrightarrow \mathbb{R}$ e é cilíndrica, ou seja, depende de um número finito de coordenadas (no nosso caso depende de um número finito de sítios).

Estudos sobre a medida invariante em passeios aleatórios em $\mathbb{Z}$ com tempo discreto podem ser encontrados em Derman (1955) e Port (1967). Especificamente, em Derman ([6]) o modelo tratado consiste de uma quantidade enumerável de partículas se movimentando sobre $\mathbb{Z}$ de acordo com uma Cadeia de Markov. Mais precisamente, denotando por $X_{n}(i)$ o número de partículas no sítio $i$ no instante $n$, suponha $X_{n}(i)$ com matriz de transição $P$ irredutível e recorrente, e também para $n$ fixo, assuma que $\left\{X_{n}(i)\right\}_{i \in \mathbb{Z}}$ são mutuamente independentes. Além disso, suponha que a configuração inicial é tal que $X_{0}(i) \sim \operatorname{Pois}(\pi(i)), \pi(i)$ a $i$-ésima componente da medida invariante $\pi$ de $P$. Então, para todo $n$ fixo, $\left\{X_{n}(i)\right\}_{i \in \mathbb{Z}}$ são independentes e cada $X_{n}(i)$ tem distribuição $\operatorname{Pois}(\pi(i))$. Isto quer dizer que começando com a medida-produto $\otimes_{i \in \mathbb{Z}}$ Pois $(\pi(i))$ o processo permanece com esta mesma ao longo do tempo.

Em relação ao modelo que estamos tratando o tempo é contínuo, ao contrário do que foi considerado por Derman ([6]). Para identificar a distribuição limite do número de partículas nos sítios começamos lembrando que cada sítio inicia, em média, com um número $\lambda$ de partículas, e estas se deslocam de acordo com um processo de Poisson de taxa 1. Seja $x \in \mathbb{Z}$. Para $y \leq x$ definimos

$$
N_{t}^{y}(x):=\text { "número de partículas que saem de } y \text { e estão em } x \text { no tempo } t \text { ". }
$$

A probabilidade de uma partícula que saiu de $y$ alcançar $x$ em um tempo $t$ é $p_{t}^{y}(x)=$ $e^{-t} \frac{t^{(x-y)}}{(x-y) !}$, e por isso, $N_{t}^{y}(x) \sim \operatorname{Pois}\left(\lambda p_{t}^{y}(x)\right)$, além de $\left\{N_{t}^{y}(x)\right\}_{y \leq x}$ serem mutuamente independentes. Portanto, o número de partículas que alcançam $x$, após decorrido um tempo $t$, é $\sum_{y \leq x} N_{t}^{y}(x) \sim \operatorname{Pois}(\lambda)$, pois $\sum_{y \leq x} \lambda p_{t}^{y}(x)=\lambda$. Analogamente, $N_{t}^{x}(z) \sim$ $\operatorname{Pois}\left(\lambda p_{t}^{x}(z)\right), z \geq x, \operatorname{com}\left\{N_{t}^{x}(z)\right\}_{z \geq x}$ mutuamente independentes. Portanto, o número 
médio de partículas que saem de $x$, após decorrido um tempo $t$, é $\sum_{z \geq x} N_{t}^{x}(z) \sim$ $\operatorname{Pois}(\lambda)$. Interpretando estes resultados podemos pensar que decorrido um tempo $t$, o número médio de partículas que chegam em $x$ é $\lambda$, da mesma forma que o número médio que sai é também $\lambda$.

Introduziremos uma modificação no modelo acima descrito. Iremos centralizar a fronteira sempre no sítio zero. Este novo modelo será chamado de modelo centrado, cuja configuração denotaremos por $\eta^{c}$. Esta modificação será útil para estudar a velocidade com que a contaminação avança à direita.

Para $Z_{t}$, fronteira atual no modelo original, a relação com o modelo centrado é

$$
\eta_{t}(x)=\eta_{t}^{c}\left(x-Z_{t}\right)+I_{\left\{x-Z_{t}=0\right\}} .
$$

Em (1.1.3), se $x=Z_{t}$, a configuração é tal que $\eta_{t}\left(Z_{t}\right)=\eta_{t}^{c}(0)+1$. Esta partícula adicional no zero é dita superpartícula. A presença da superpartícula será apenas no sítio zero, pois para $x \neq Z_{t}, \eta_{t}(x)=\eta_{t}^{c}\left(x-Z_{t}\right)$.

A partir daqui trabalharemos apenas com o modelo centrado. Sempre que for necessário distinguir os dois modelos usaremos a notação $\eta_{t}^{c}$. Também, sempre que não for necessário distinguir o instante atual usaremos $\eta$ ao invés de $\eta_{t}$.

Cada vez que uma partícula do sítio zero saltar à direita será realizada uma translação para trás (à esquerda), ou seja, a configuração de cada sítio é deslocada para o vizinho da esquerda, e assim, a fronteira voltará novamente a ser o zero. Intuitivamente, a fronteira permanecerá a mesma durante a evolução do processo. Podemos ainda dizer que a fronteira "quer" se deslocar com taxa $1+\eta^{c}(0)$, mas quando o evento ocorre, o zero volta a ser a fronteira.

Formalizamos o modelo centrado, definindo o respectivo gerador:

Definição 1.1.2. O gerador infinitesimal do processo centrado é definido como 


$$
G f(\eta):=\sum_{x \neq 0} \eta(x)\left(f\left(\eta^{x, x+1}\right)-f(\eta)\right)+(\eta(0)+1)(f(\stackrel{\leftarrow}{\eta})-f(\eta))
$$

$\eta^{x, x+1}$ definida em (1.1.2). Em (1.1.4), notamos que para $x=0$ a configuração é $\eta(0)+1$. Isto está de acordo com o conceito de superpartícula antes mencionado. A função $f$ é tal que $f: \mathbb{N}^{\mathbb{Z}} \longrightarrow \mathbb{R}$ e é cilíndrica. A configuração $\stackrel{\leftarrow}{\eta}$ é tal que $\stackrel{\leftarrow}{\eta}(x)=\eta(x+1)$, para $x \in \mathbb{Z}$. O operador $\leftarrow$ é dito operador translação.

O quociente $\frac{Z_{t}}{t}$ é a posição da fronteira atual pelo comprimento do intervalo de tempo, enquanto $v:=\lim _{t \rightarrow \infty} \frac{Z_{t}}{t}$ é a velocidade de contaminação do modelo original. No artigo de Kesten ([13]) foi provada a existência da velocidade para um modelo análogo.

Queremos estabelecer uma relação entre a velocidade do modelo original e a do centrado. Seja $0<h<1$. Como $Z_{0}=0$,

$$
\frac{Z_{t}}{t}=\frac{\frac{1}{h}\left(Z_{t}-Z_{0}\right)}{\frac{t}{h}}=\frac{\frac{1}{h}\left(Z_{t}-Z_{t-h}\right)+\frac{1}{h}\left(Z_{t-h}-Z_{t-2 h}\right)+\cdots+\frac{1}{h}\left(Z_{h}-Z_{0}\right)}{\frac{t}{h}} .
$$

Denote $\xi_{i}:=\frac{1}{h}\left(Z_{t-h(i-1)}-Z_{t-h i}\right), i=1, \ldots, \frac{t}{h}$, e suponha que a seqüência $\left\{\xi_{1}, \xi_{2}, \ldots\right\}$ seja estacionária ergódica com $E \xi_{1}$ finita. Pelo Teorema Ergódico de Birkhoff,

$$
\lim _{t \rightarrow \infty} \frac{\xi_{1}+\xi_{2}+\cdots+\xi_{\frac{t}{h}}}{\frac{t}{h}}=E \xi_{1}, \quad \text { quase certamente. }
$$

Decorre, da convergência quase certa acima, que para $t$ suficientemente grande 


$$
\begin{aligned}
\frac{Z_{t}}{t} & \approx E \xi_{1} \\
& =E\left(\frac{1}{h}\left(Z_{t}-Z_{t-h}\right)\right) \\
& =E\left(\frac{1}{h}\left(Z_{t+h}-Z_{t}\right)\right) \\
& =E\left(E\left(\frac{1}{h}\left(Z_{t+h}-Z_{t}\right) \mid \eta_{t}\left(Z_{t}\right)\right)\right) .
\end{aligned}
$$

Tomando $h$ pequeno e observando que no modelo original $Z_{t}$ se desloca para o sítio da direita com taxa $\eta_{t}\left(Z_{t}\right)$,

$$
E\left(\frac{1}{h}\left(Z_{t+h}-Z_{t}\right) \mid \eta_{t}\left(Z_{t}\right)=m\right)=\frac{1}{h} \times(m h)+0 \times(1-m h)=m
$$

ou seja, $E \xi_{1}=E \eta_{t}\left(Z_{t}\right)$. Voltando a (1.1.5) e utilizando (1.1.3),

$$
\frac{Z_{t}}{t} \approx E \eta_{t}\left(Z_{t}\right)=E \eta_{t}^{c}(0)+1=E \eta^{c}(0)+1
$$

onde $E \eta_{t}^{c}(0)=E \eta^{c}(0)$, uma vez que podemos supor que o processo centrado está no regime estacionário. Segue que

$$
\lim _{t \rightarrow \infty} \frac{Z_{t}}{t}=1+E \eta^{c}(0)
$$

De acordo com o raciocínio acima deduzimos que $v=1+E \eta^{c}(0)$. No processo original essa velocidade significa que, em média, uma partícula da fronteira avança $1+E^{c} \eta(0)$ por unidade de tempo. No processo centrado é o número médio de partículas que estão no zero (que sempre é a fronteira).

A definição de velocidade é tal que leva em consideração apenas a fronteira atual e a definição de $Z_{t}$ não especifica qual partícula irá saltar, ou seja, o conceito de fronteira não faz distinção entre as partículas, mas leva em consideração apenas a posição em $\mathbb{Z}$ no instante $t$. Mas, vamos nos concentrar em uma partícula específica, 
a qual chamaremos de partícula marcada, e deduzir a respectiva velocidade com que ela se desloca. Denotaremos por $v_{m}:=\lim _{t \rightarrow \infty} \frac{N_{t}}{t}$ a velocidade do ponto de vista da partícula marcada, com $N_{t}$ representando a posição desta partícula no instante $t$. Uma vez que definimos o modelo de maneira que cada partícula se desloca segundo um Processo de Poisson de intensidade 1, então $\left\{N_{t}\right\}_{t \geq 0}$ é um Processo de Poisson com esta intensidade.

Lembremos a desigualdade de Doob para submartingal não negativo. Seja $\left\{X_{t}\right\}_{t \geq 0}$ submartingal. Para $p>1$,

$$
E\left(\sup _{\sigma \leq t \leq \tau} X_{t}\right)^{p} \leq\left(\frac{p}{p-1}\right)^{p} E\left(X_{\tau}^{p}\right)
$$

desde que $X_{t} \geq 0$ para todo $t$, quase certamente, e $E\left(X_{\tau}^{p}\right)<\infty$. Mas $\left\{N_{t}-t\right\}_{t \geq 0}$ é martingal. Para verificar isto começamos obsevando que $\left\{M_{t}\right\}_{t \geq 0}, M_{t}=N_{t}-t$, é tal que $E M_{t}=0$ e tem incrementos independentes e estacionários. Por estes fatos,

$$
E\left(M_{t} \mid \mathcal{F}_{s}^{M}\right)=E\left(M_{s} \mid \mathcal{F}_{s}^{M}\right)+E\left(\left(M_{t}-M_{s}\right) \mid \mathcal{F}_{s}^{M}\right)=M_{s}+E\left(M_{t}-M_{s}\right)=M_{s}
$$

$\mathcal{F}_{s}^{M}:=\sigma\left(M_{s}: s \in[0, t]\right)$ a $\sigma$ - álgebra gerada pela família $\left\{M_{s}: s \in[0, t]\right\}$.

Em (1.1.6) tome $\sigma=2^{n}, \tau=2^{n+1}$ e $p=2$. Assim,

$$
\begin{aligned}
E\left(\sup _{2^{n} \leq t \leq 2^{n+1}}\left(\frac{N_{t}-t}{t}\right)^{2}\right) & \leq \frac{1}{2^{2 n}} E\left(\sup _{2^{n} \leq t \leq 2^{n+1}}\left(N_{t}-t\right)^{2}\right) \\
& \leq \frac{1}{2^{2 n}} 4 E\left(N_{\tau}-\tau\right)^{2} \\
& =\frac{4}{2^{2 n}} 2^{n+1} \\
& =\frac{8}{2^{n}}
\end{aligned}
$$

Usando a desigualdade de Chebyshev e (1.1.7), 


$$
\begin{aligned}
P\left(\sup _{2^{n} \leq t \leq 2^{n+1}}\left|\frac{N_{t}}{t}-1\right|>\epsilon\right) & =P\left(\sup _{2^{n} \leq t \leq 2^{n+1}}\left(\frac{N_{t}-t}{t}\right)^{2}>\epsilon^{2}\right) \\
& \leq \frac{1}{\epsilon^{2}} E\left(\sup _{2^{n} \leq t \leq 2^{n+1}}\left(\frac{N_{t}-t}{t}\right)^{2}\right) \\
& \leq \frac{8}{\epsilon^{2} 2^{n}} .
\end{aligned}
$$

Por (1.1.8) segue que $\frac{N_{t}}{t}$ converge em probabilidade para 1 quando $t \rightarrow \infty$. Ainda por (1.1.8), como $\sum_{n=1}^{\infty} \frac{8}{\epsilon^{2} 2^{n}}<\infty, \lim _{t \rightarrow \infty} \frac{N_{t}}{t}=1$ quase certamente. Concluimos que $v_{m}=1$ quase certamente. A diferença $v-v_{m}$ será denominada de velocidade de contaminação com relação à partícula marcada. Note que $v-v_{m}=E \eta^{c}(0)$ quase certamente. Na seção 1.7 voltaremos a nos referir à diferença $v-v_{m}$.

\subsection{O algoritmo para a simulação}

Um dos objetivos será estimar a velocidade $v$. Para tal fim faremos simulações. Nestas simulações consideraremos uma caixa de tamanho $2 N$, isto é, os sítios $\{-N, \ldots, 0, \ldots, N\}$, e um tempo máximo $T$ de simulação. Na evolução da simulação, a cada iteração, um sítio será escolhido segundo a lei de probabilidade

$$
P(\text { sítio } x \text { ser selecionado })=\frac{\text { número de partículas no sítio } x}{\text { número de partículas na caixa }} \text {. }
$$

Neste sítio $x$ apenas uma partícula saltará para $x+1$. Se caso $x=0$, então será realizada uma translação à esquerda, que consiste em deslocar as partículas de $x$ para $x-1$, isto para todo $x$ na caixa.

A seguir faremos o detalhamento do algoritmo para a simulação do modelo centrado.

(1o) Fixar $N$ inteiro positivo. Isto significa que o número de sítios será finito, ou seja, ficaremos restritos ao conjunto $\{-(N+1),-N, \ldots,-1,0,1, \ldots, N\}$. Introduzimos o 
sítio - $(N+1)$ para que durante a simulação haja um número satisfatório de partículas no sistema para um longo tempo. Também fixe $\lambda>0$ (o parâmetro da distribuição de Poisson) e $T>0$ o tempo máximo da simulação.

(2o) $t \longleftarrow 0$, que é o instante inicial.

(3o) No instante inicial cada sítio recebe uma quantidade de partículas segundo uma distribuição de Poisson de parâmetro $\lambda$. Esses valores gerados serão alocados no vetor Eta, que terá $2 N+2$ componentes.

(40) Sítios $\{-(N+1),-N, \ldots,-1,0\}$ serão declarados contaminados e $\{1,2, \ldots, N\}$ normais.

(5o) $S_{t} \longleftarrow \sum_{i=-N-1}^{N} E t a(i)$

(6o) Selecione aleatoriamente um sítio segundo a distribuição de probabilidade

$$
P\left(X_{t}=i\right)=\frac{E t a(i)}{S_{t}}
$$

$i \in\{-(N+1),-N, \ldots,-1,0,1, \ldots, N\}$, sendo $X_{t}$ a variável aleatória correspondendo ao sítio escolhido no instante $t$. Denote por $x_{t}$ o sítio selecionado.

(7o)

$$
\operatorname{Eta}\left(x_{t}\right) \longleftarrow \begin{cases}E t a(-N)+1, & \text { se } x_{t}=-(N+1) \\ \operatorname{Eta}(N)-1, & \text { se } x_{t}=N\end{cases}
$$

$\operatorname{Eta}\left(x_{t}\right) \longleftarrow \operatorname{Eta}\left(x_{t}\right)-1$ e $\operatorname{Eta}\left(x_{t}+1\right) \longleftarrow \operatorname{Eta}\left(x_{t}+1\right)+1$, se $x_{t} \neq\{-(N+1), N\}$.

(8o) Calcule as estatísticas para estimar $E(\eta(x))$ e $\operatorname{Cov}(\eta(x), \eta(y))$ e eventualmente a lei de $\eta(x)$. Para o sítio $-(N+1)$ não serão feitas estatísticas. 
(9o) Se $x_{t}=0$ faça a translação, isto é,

$$
\operatorname{Eta}(y) \longleftarrow \operatorname{Eta}(y+1), y \in\{-N, \ldots, N-1\},
$$

e além disso, $\operatorname{Eta}(N) \longleftarrow \operatorname{Pois}(\lambda)$. Observe que quando realizamos a translação o último sítio(N) perde sua configuração. É por isso que geramos um número segundo uma $\operatorname{Pois}(\lambda)$ e o atribuimos a $\operatorname{Eta}(N)$.

(10o) Gere um tempo $\tau$ segundo uma exponencial de parâmetro $S_{t}$ e faça $t=t+\tau$.

(11o) Se $t \leq T$ então $E t a(-N-1) \longleftarrow \operatorname{Pois}(\lambda)$ e volte para (5ó), senão calcule as estimativas de $E(\eta(x))$ e $\operatorname{Cov}(\eta(x), \eta(y))$. Neste passo geramos um número aleatório para $E t a(-N-1)$ com o objetivo de que haja a entrada de $\lambda$ partículas, em média, em cada iteração.

\subsection{Programa em Scilab}

Após a listagem do programa, encontram-se as instruções de como executá-lo.

Listagem do programa em Scilab para simulação do modelo centrado:

clear all;

$o p=1 ; / /$ define o tipo de visao: local $(o p=1)$ e panoramica(op=2)

$\mathrm{N}=50 ; / /$ parametro para tamanho da caixa

$\mathrm{n}=10 ; / /$ amostra dos sitios

$\mathrm{T}=1000 ; / /$ tempo final da simulacao

lambda $1 ;$ //parametro da distribuicao inicial 


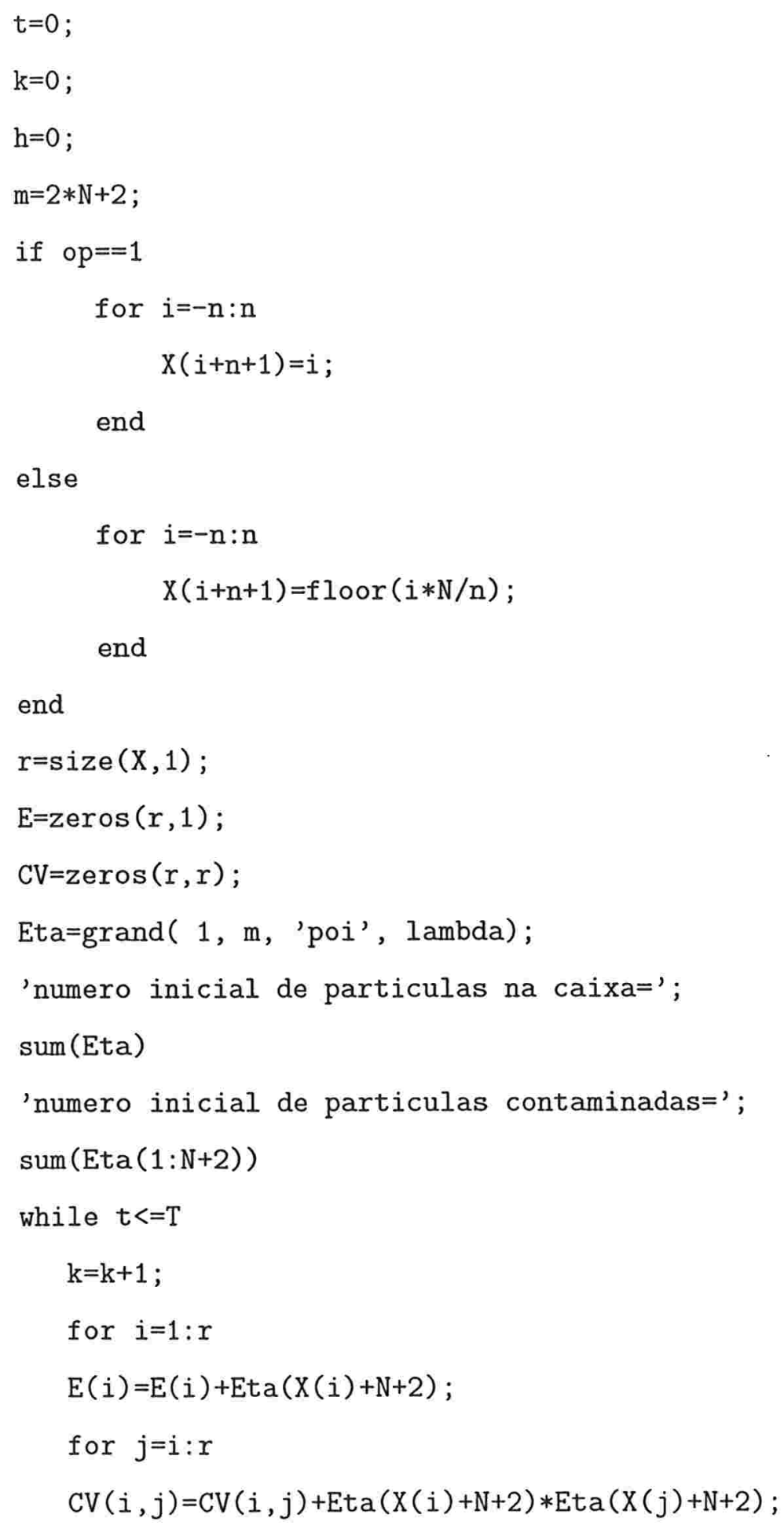




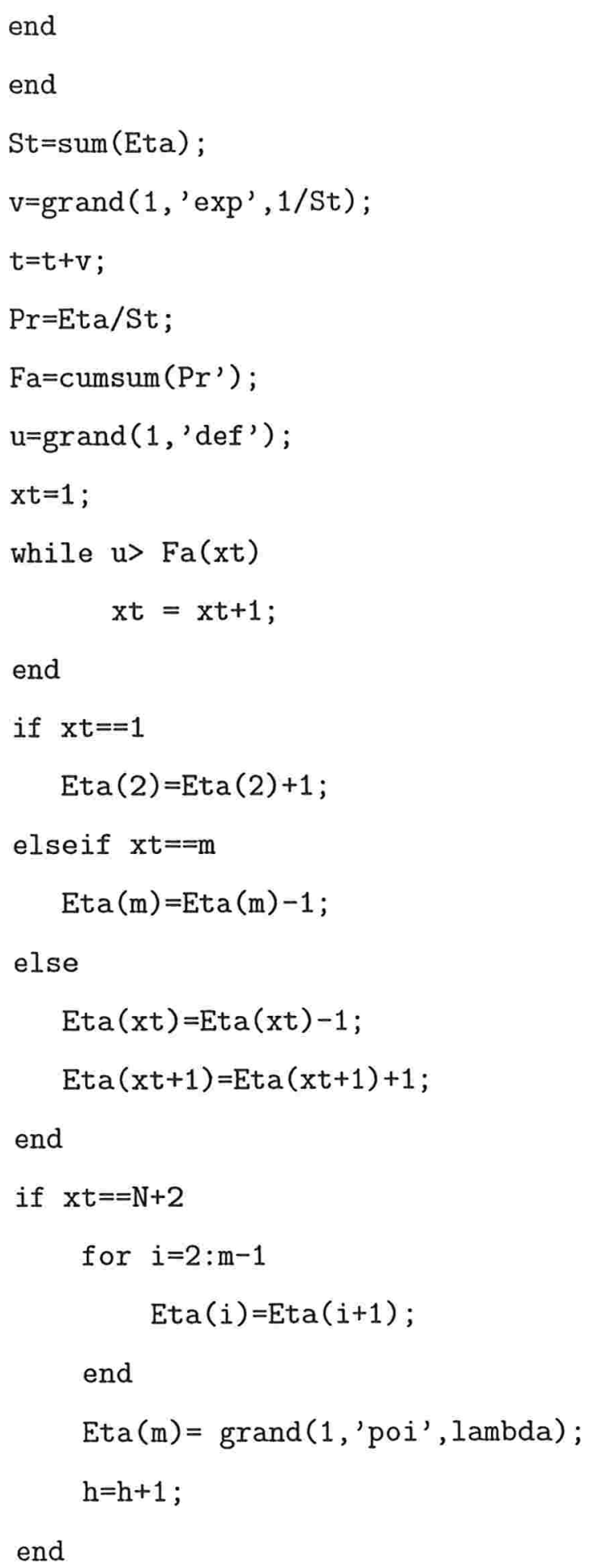




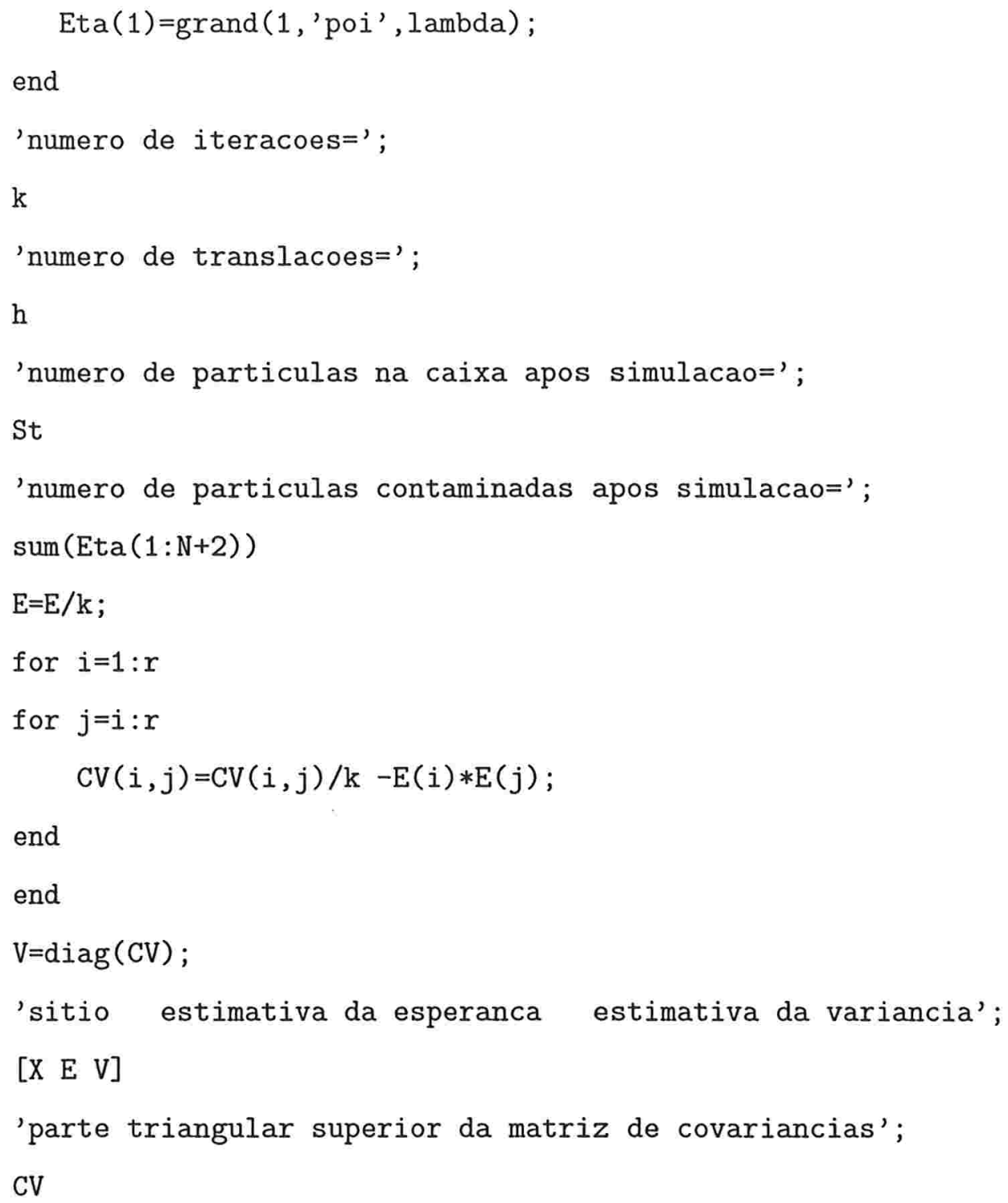

Para utilizar o programa somente as variáveis op, $\mathrm{N}, \mathrm{n}, \mathrm{T}$ e lambda devem ter valores atribuídos pelo usuário. Observe que o programa não permite " $\lambda$ " como nome de variável, logo, a variável no programa correspondente a este parâmetro é lambda. Mesma justificativa vale para o uso de Eta como variável no programa, no lugar de " $\eta$ ". 
A variável op=1 significa que será considerada uma "visão local", onde serão impressos os sítios

$$
\{-n, \ldots,-1,0,1, \ldots, n\} \text {. }
$$

Se op=2 então será considerada uma "visão panorâmica", onde serão impressos os sítios

$$
\left\{\left\lfloor i \frac{N}{n}\right\rfloor: i=-n, \ldots,-1,0,1, \ldots, n\right\} .
$$

A variável $\mathrm{N}$ é o parâmetro para o tamanho da caixa, que é um intervalo de amplitude $2 \mathrm{~N}$, e $\mathrm{n}<\mathrm{N}$ é tal que $2 \mathrm{n}+1$ sítios serão apresentados. T é o tempo máximo de simulação e lambda o parâmetro da distribuição de Poisson.

Após o término da execução o programa fornece:

- número inicial de partículas na caixa;

- número inicial de partículas contaminadas;

- número de iterações;

- número de translações;

- número de partículas na caixa após a simulação;

- número de partículas contaminadas após a simulação;

- estimativa da esperança de $\eta(x)$;

- matriz de estimativas de covariâncias e variâncias de $\eta$. Na diagonal principal estão as variâncias e acima as covariâncias.

O número de iterações se refere à quantidade de partículas que se deslocaram para o vizinho da direita. Também, o número de iterações é o tamanho da amostra para estimar as esperanças e covariâncias para $\eta$. 
Devido à definição de superpartícula, anteriormente mencionada, no sítio zero teremos $1+\hat{E}(\eta(0))$, sendo $\hat{E}(\eta(0))$ a estimativa de $E \eta(0)$. Portanto, $1+\hat{E}(\eta(0))$ será a estimativa de $v$.

\subsection{Alguns exemplos da execução do programa}

Na primeira execução do programa utilizamos:

$\mathrm{op}=1$

$\mathrm{N}=50$

$n=10$

$\mathrm{T}=2000$

lambda $=1$.

Os resultados foram:

número inicial de partículas na caixa=101

número inicial de partículas contaminadas $=52$

número de iterações $=207861$

número de translações $=3242$

número de partículas na caixa após a simulação=98

número de partículas contaminadas após a simulação=50.

A velocidade de contaminação estimada é obtida na Tabela 1.1, na linha referente ao sítio zero. Nesta simulação a estimativa de $v$ foi 1.6343.

O número esperado de partículas no instante inicial é $\lambda(2 N+2)$. Disso decorre que os tempos exponenciais gerados têm média $\frac{1}{\lambda(2 N+2)}$, e assim, o número esperado de iterações é $T \lambda(2 N+2)$. Com relação ao número esperado de translações, este é 
aproximadamente $\left(\frac{1+\hat{E} \eta(0)}{\lambda(2 N+2)}\right) \times$ (número esperado de iterações), pois $\frac{1+\hat{E} \eta(0)}{\lambda(2 N+2)}$ é a probabilidade estimada do sítio zero ser escolhido numa iteração. Na simulação anterior o número esperado de iterações é $2000 \times 1 \times(2 \times 50+2)=204000$ e o de translações $\left(\frac{1+0.6343}{1 \times(2 \times 50+2)}\right) \times 204000=3268.59$. 


\begin{tabular}{|r|c|c|}
\hline sítio & estimativa da esperança & estimativa da variância \\
\hline-10 & 1.0089 & 1.0860 \\
-9 & 0.9813 & 1.1093 \\
-8 & 1.0209 & 1.0534 \\
-7 & 0.9998 & 0.9547 \\
-6 & 1.0157 & 0.9903 \\
-5 & 1.0263 & 1.0584 \\
-4 & 1.0111 & 1.0911 \\
-3 & 1.0349 & 1.0808 \\
-2 & 1.0437 & 0.9974 \\
-1 & 1.0557 & 0.9650 \\
0 & 1.6343 & 0.6308 \\
1 & 0.4139 & 0.4114 \\
2 & 0.6682 & 0.6846 \\
3 & 0.7924 & 0.8224 \\
4 & 0.8799 & 0.8524 \\
5 & 0.9570 & 0.9062 \\
6 & 0.9809 & 1.0194 \\
7 & 1.0061 & 1.0338 \\
8 & 0.9945 & 1.0078 \\
9 & 1.0034 & 0.9802 \\
10 & 1.0196 & 1.0902 \\
\hline
\end{tabular}

Tabela 1.1: Resultados da simulação para $\lambda=1 \mathrm{e} o \mathrm{p}=1$ 
A matriz abaixo se refere às estimativas das covariâncias e variâncias para $\eta(x)$, $x \in\{-4,-3, \ldots, 0, \ldots, 3,4\}$. Na diagonal principal temos as variâncias.

$$
\begin{array}{rrrrrrr}
1.0911 & -0.0197 & 0.0199 & 0.0292 & 0.0428 & -0.0001 & -0.0224 \\
& 1.0808 & -0.0330 & -0.0071 & -0.0244 & -0.0146 & -0.0140 \\
& & 0.9974 & -0.0431 & -0.0045 & -0.0325 & 0.0094 \\
& & & 0.9650 & -0.0557 & -0.0406 & -0.0604 \\
& & & & 0.6380 & -0.0330 & -0.0076 \\
& & & & & 0.4114 & 0.0561 \\
& & & & & & 0.6846
\end{array}
$$

$\left.\begin{array}{rr}-0.0453 & 0.0115 \\ -0.0567 & -0.0729 \\ 0.0063 & -0.0078 \\ -0.0317 & 0.0209 \\ 0.0078 & -0.0202 \\ 0.0381 & 0.0376 \\ 0.0536 & 0.0130 \\ 0.8224 & 0.0037 \\ & 0.8524\end{array}\right)$


Também executamos o programa com $o p=2, N=50, n=10$, lambda=1 e $T=2000$, cujos resultados foram:

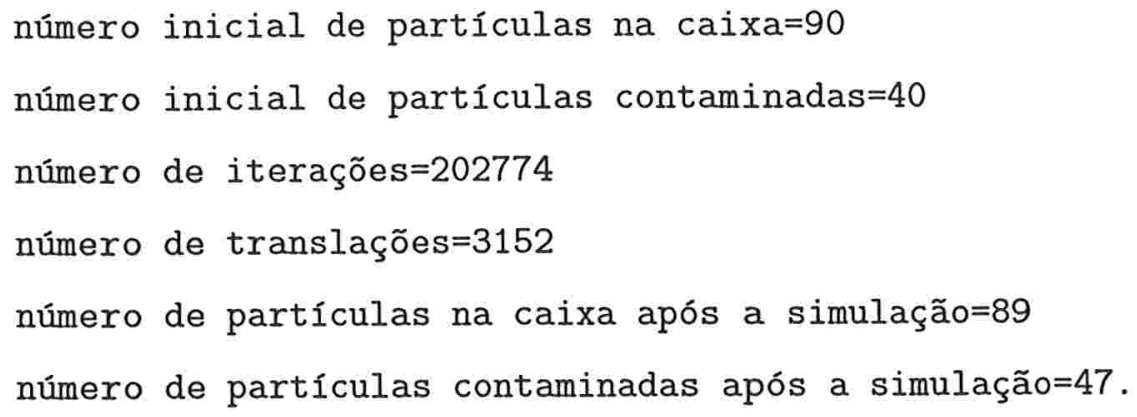

Na Tabela 1.2 observamos que a velocidade estimada foi 1.6032 .

Outra simulação foi com os seguintes valores:

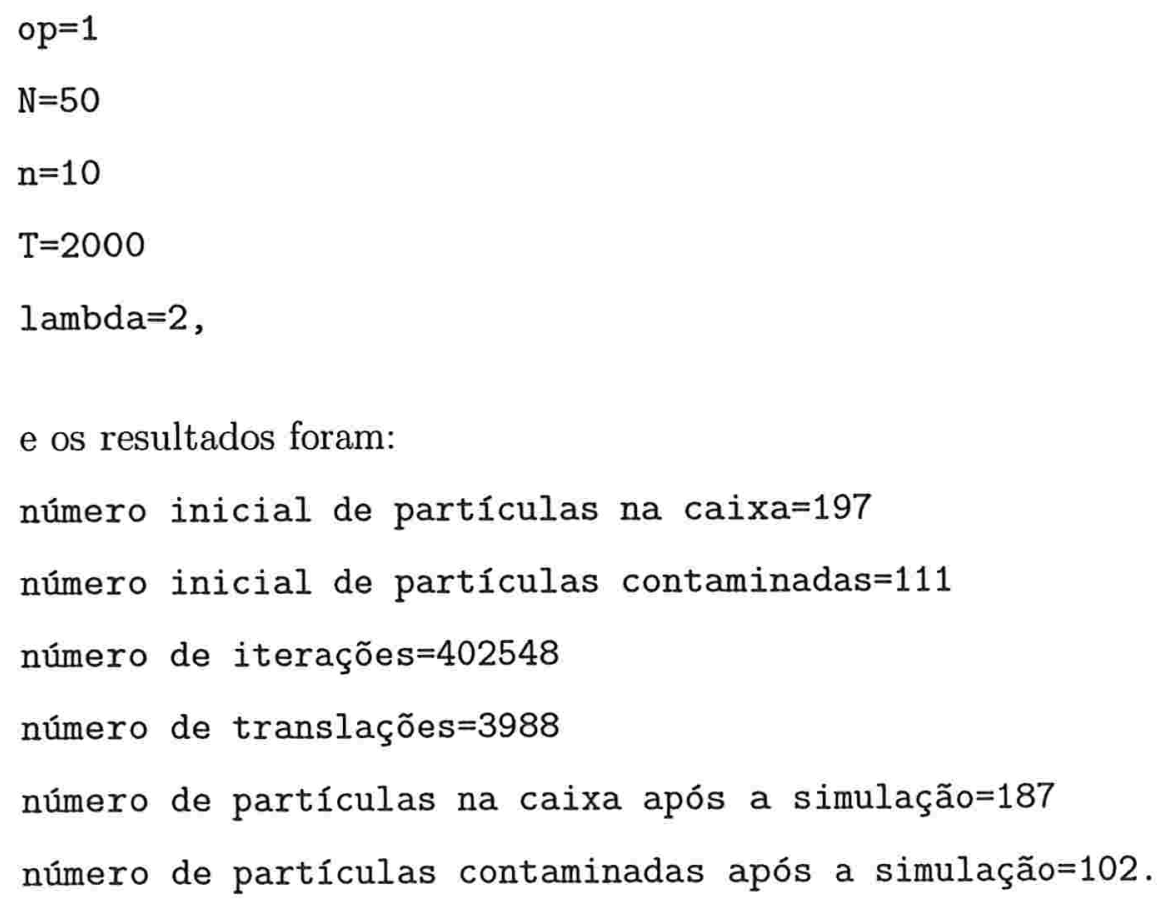

A estimativa da velocidade é obtida na Tabela 1.3, que é 2.3844 . 


\begin{tabular}{|c|c|c|}
\hline sítio & estimativa da esperança & estimativa da variância \\
\hline-50 & 0.9821 & 1.0115 \\
\hline-45 & 0.9772 & 0.9786 \\
\hline-40 & 1.0069 & 0.9890 \\
\hline-35 & 0.9425 & 0.9193 \\
\hline-30 & 1.0179 & 1.0096 \\
\hline-25 & 1.1349 & 1.0473 \\
\hline-20 & 0.9911 & 0.9473 \\
\hline-15 & 1.0575 & 0.9649 \\
\hline-10 & 1.0208 & 1.0402 \\
\hline-5 & 1.0625 & 1.0248 \\
\hline 0 & 1.6032 & 0.6996 \\
\hline 5 & 0.8214 & 0.8550 \\
\hline 10 & 1.0119 & 1.0078 \\
\hline 15 & 1.1974 & 1.3073 \\
\hline 20 & 1.1379 & 1.1169 \\
\hline 25 & 1.0724 & 1.0692 \\
\hline 30 & 0.9446 & 0.8991 \\
\hline 35 & 0.9048 & 0.8639 \\
\hline 40 & 0.9970 & 0.9236 \\
\hline 45 & 1.1517 & 1.1942 \\
\hline 50 & 1.1006 & 1.1247 \\
\hline
\end{tabular}

Tabela 1.2: Resultados da simulação para $\lambda=1 \mathrm{e} o \mathrm{p}=2$ 


\begin{tabular}{|c|c|c|}
\hline sítio & estimativa da esperança & estimativa da variância \\
\hline-10 & 2.0095 & 2.0185 \\
\hline-9 & 1.9957 & 1.9759 \\
\hline-8 & 2.0146 & 2.0759 \\
\hline-7 & 1.9612 & 2.1198 \\
\hline-6 & 2.0053 & 2.0323 \\
\hline-5 & 2.0181 & 2.0063 \\
\hline-4 & 2.0343 & 2.0277 \\
\hline-3 & 2.0025 & 2.1821 \\
\hline-2 & 2.0246 & 2.0166 \\
\hline-1 & 2.0362 & 1.8519 \\
\hline 0 & 2.3844 & 1.4671 \\
\hline 1 & 1.1655 & 1.2948 \\
\hline 2 & 1.6484 & 1.6459 \\
\hline 3 & 1.8522 & 1.7685 \\
\hline 4 & 1.9612 & 1.8694 \\
\hline 5 & 1.9800 & 1.8475 \\
\hline 6 & 2.0246 & 1.9692 \\
\hline 7 & 1.9918 & 2.0703 \\
\hline 8 & 2.0186 & 2.0365 \\
\hline 9 & 2.0444 & 2.0601 \\
\hline 10 & 2.0561 & 2.0128 \\
\hline
\end{tabular}

Tabela 1.3: Resultados da simulação para $\lambda=2$ e op=1 
Também apresentamos a matriz de estimativas de covariâncias e variâncias para $\eta(x), x \in\{-4,-3, \ldots, 0, \ldots, 3,4\}$, na simulação com $\lambda=2$.

$$
\begin{aligned}
& \begin{array}{|rrrrrrr}
2.0277 & 0.0470 & 0.0213 & -0.0215 & -0.0187 & 0.0522 & 0.0872 \\
& 2.1821 & 0.1123 & 0.0192 & 0.0432 & -0.0484 & -0.0281 \\
& & 2.0166 & 0.0391 & 0.0199 & 0.0119 & 0.0202 \\
& & & 1.8519 & -0.0207 & -0.0485 & -0.0610 \\
& & & & 1.4671 & 0.0250 & 0.0695 \\
& & & & & 1.2948 & 0.1666 \\
& & & & & & 1.6459
\end{array} \\
& \left.\begin{array}{rr}
0.0835 & 0.1174 \\
-0.0410 & 0.0409 \\
0.0030 & 0.0489 \\
0.0103 & 0.0194 \\
0.0154 & -0.0483 \\
0.0553 & 0.0194 \\
0.0678 & 0.0659 \\
1.7685 & 0.0530 \\
& 1.8694
\end{array}\right)
\end{aligned}
$$

\subsection{Sobre a medida invariante do modelo centrado}

De acordo com [18] (Teorema B7), sabe-se que $\mu$ é medida invariante do processo se e somente se

$$
E_{\mu} G f(\eta)=0,
$$

para todas funções cilíndricas $f$, onde $E_{\mu}$ é a esperança com relação à medida $\mu$.

Seja $f(\eta)=\eta(1)+\eta(2)+\cdots+\eta(n), n$ inteiro positivo. Por (1.1.4), 


$$
\begin{aligned}
G f(\eta) & =\eta(n)[\eta(1)+\eta(2)+\cdots+\eta(n)-1-\eta(1)-\eta(2)-\cdots-\eta(n)] \\
& +(\eta(0)+1)[\eta(2)+\cdots+\eta(n)+\eta(n+1) \\
& -\eta(1)-\eta(2)-\cdots-\eta(n)] \\
& =-\eta(n)+(\eta(0)+1)[\eta(n+1)-\eta(1)] .
\end{aligned}
$$

De (1.5.1) e (1.5.2),

$$
-E_{\mu} \eta(n)+E_{\mu}(\eta(0) \eta(n+1))-E_{\mu}(\eta(0) \eta(1))+E_{\mu} \eta(n+1)-E_{\mu} \eta(1)=0 .
$$

Observando as Figuras 1.1 e 1.2 percebemos que o comportamento das estimativas das esperanças tem uma queda ("um poço") no sítio 1, e no sítio zero um salto, ficando acima de $\lambda$. Por outro lado, para sítios negativos as esperanças não se desviam muito de $\lambda$, assim como para sítios positivos distantes do zero. Essa queda no sítio 1 tem uma explicação. Empiricamente, $\lim _{n \rightarrow \infty} E_{\mu} \eta(n)=\lambda$, assim como $\eta(0)$ e $\eta(n+1)$ podem ser assumidos como independentes para $n$ grande. Logo, pela equação (1.5.3),

$$
\lambda E_{\mu} \eta(0)-E_{\mu}(\eta(0) \eta(1))-E_{\mu} \eta(1)=0 .
$$

Segue que

$$
E_{\mu} \eta(1)=\lambda E_{\mu} \eta(0)-E_{\mu}(\eta(0) \eta(1))<\lambda E_{\mu} \eta(0)
$$

Como veremos ainda nesta seção, para $\lambda$ pequeno, $E_{\mu} \eta(0) \approx \frac{\lambda}{2}$, e portanto, $E_{\mu} \eta(1) \approx$ $O\left(\lambda^{2}\right)$.

O fato do processo iniciar com a medida-produto de $\operatorname{Pois}(\lambda)$ não significa que também a teremos como medida invariante. Adiante apresentaremos um cálculo, o qual mostra que a medida-produto de $\operatorname{Pois}(\lambda)$ falha como medida invariante (não 


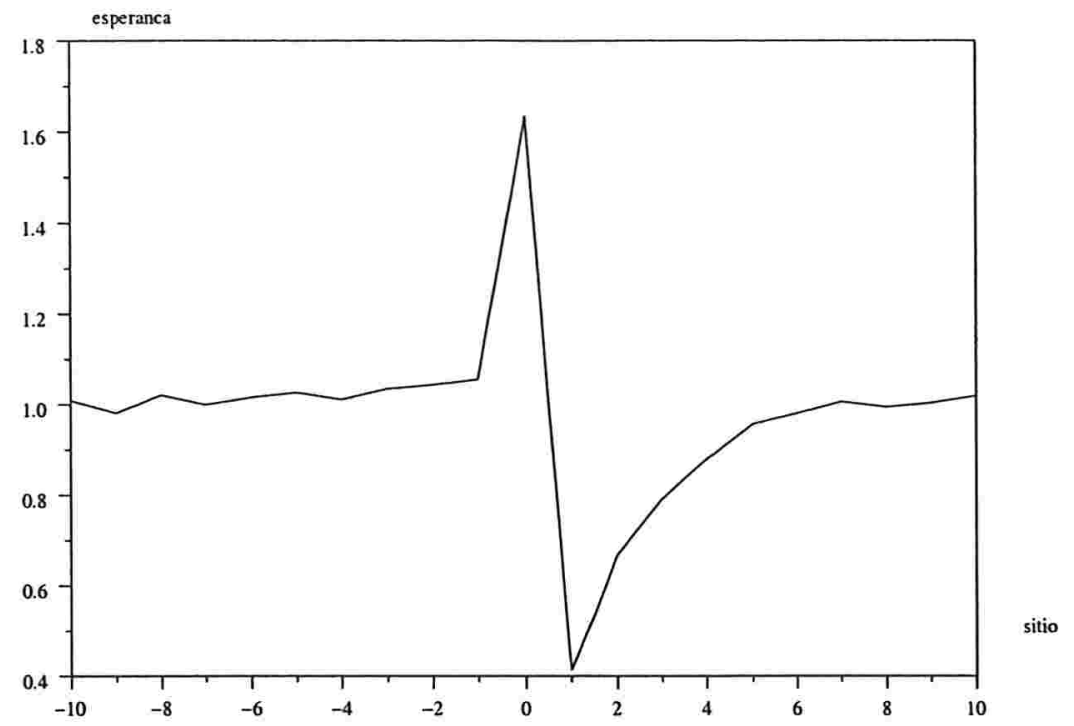

Figura 1.1: Esperanças estimadas para $\lambda=1$

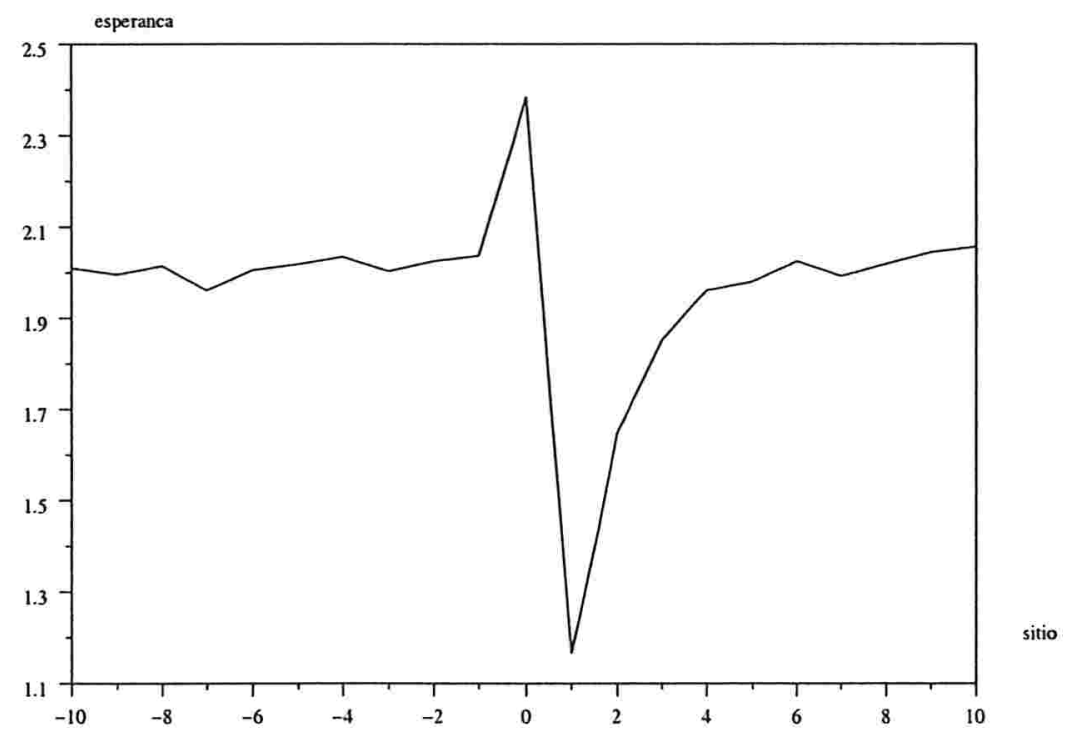

Figura 1.2: Esperanças estimadas para $\lambda=2$ 
satisfaz (1.5.1)). Mas, por um momento, vamos admitir que a medida-produto seja a invariante e verifiquemos que forma terá $E \eta(n)$. Pela hipótese momentânea de que vale (1.5.1) para a medida-produto, e denotando $a:=E_{\mu} \eta(0)$, a equação (1.5.3) fica

$$
\left.-E_{\mu} \eta(n)+(1+a) E_{\mu} \eta(n+1)\right)-(1+a) E_{\mu} \eta(1)=0 .
$$

Pelo fato de $E_{\mu} \eta(n)=E_{\mu} \eta(n+1)=\lambda$ para $n$ grande, por (1.5.4),

$$
E_{\mu} \eta(1)=\lambda\left(\frac{a}{1+a}\right)=\lambda\left(1-\frac{1}{1+a}\right) .
$$

Com $n=1$ em (1.5.4), temos

$$
E_{\mu} \eta(2)=\lambda\left(\frac{a}{1+a}\right)\left(\frac{2+a}{1+a}\right) .
$$

Já, com $n=2$, obtemos

$$
E_{\mu} \eta(3)=\lambda\left(\frac{a}{1+a}\right)\left(\frac{2+a+(1+a)^{2}}{(1+a)^{2}}\right)
$$

e para $n=3$,

$$
E_{\mu} \eta(4)=\lambda\left(\frac{a}{1+a}\right)\left(\frac{2+a+(1+a)^{3}+(1+a)^{2}}{(1+a)^{3}}\right) .
$$

Recursivamente, obtemos que

$$
\begin{aligned}
E_{\mu} \eta(n) & =\lambda\left(\frac{a}{1+a}\right)\left(\frac{2+a+\sum_{k=2}^{n-1}(1+a)^{k}}{(1+a)^{n-1}}\right) \\
& =\lambda\left(\frac{a}{1+a}\right)\left(\frac{2+a}{(1+a)^{n-1}}+\frac{(1+a)^{n}-(1+a)^{2}}{a(1+a)^{n-1}}\right) \\
& =\lambda\left(1+\frac{a(2+a)-(1+a)^{2}}{(1+a)^{n}}\right) \\
& =\lambda\left(1-\frac{1}{(1+a)^{n}}\right) .
\end{aligned}
$$

Portanto, 


$$
E_{\mu} \eta(n)=\lambda\left(1-\frac{1}{(1+a)^{n}}\right), \text { para } n \geq 1
$$

Note que em (1.5.5), $\lim _{n \rightarrow \infty} E_{\mu} \eta(n)=\lambda$.

A fim de obter uma expressão para $a=E_{\mu} \eta(0)$ faremos $f(\eta)=\eta(0)$. Então, o gerador fica

$$
G f(\eta)=\eta(-1)+\eta(0) \eta(1)+\eta(1)-\eta(0)^{2}-\eta(0) .
$$

Aplicando esperança com relação à medida invariante $\mu$ em (1.5.6), e por (1.5.1), teremos

$$
E_{\mu} \eta(-1)+E_{\mu}(\eta(0) \eta(1))+E_{\mu} \eta(1)-E_{\mu}\left(\eta(0)^{2}\right)-E_{\mu} \eta(0)=0 .
$$

Empiricamente os resultados das simulações parecem sugerir que $\eta(i) \sim \operatorname{Pois}(\nu(i))$, com

$$
\nu(i)=\left\{\begin{array}{ll}
\lambda, & \text { se } i \leq-1 \\
a, & \text { se } i=0 \\
\lambda\left(1-\frac{1}{(1+a)^{i}}\right), & \text { se } i \geq 1
\end{array} .\right.
$$

Pela suposição de independência entre $\eta(0)$ e $\eta(1)$ (lembre que momentaneamente estamos supondo isto) e por (1.5.8), a equação (1.5.7) fica

$$
\begin{aligned}
\nu(-1)+\nu(0) \nu(1)+\nu(1)-\left[\operatorname{Var}(\eta(0))+\nu(0)^{2}\right]-\nu(0) & =0 \\
\lambda+a \lambda\left(\frac{a}{1+a}\right)+\lambda\left(\frac{a}{1+a}\right)-\left(a+a^{2}\right)-a & =0 \\
a^{2}-a(\lambda-2)-\lambda & =0 .
\end{aligned}
$$

Resolvendo a equação em (1.5.9), a raiz positiva será 


$$
a=\frac{\lambda-2+\sqrt{\lambda^{2}+4}}{2} .
$$

De (1.5.10), obtemos que a velocidade será

$$
v=1+\frac{\lambda-2+\sqrt{\lambda^{2}+4}}{2}=\frac{\lambda+\sqrt{\lambda^{2}+4}}{2} .
$$

Para $\lambda$ pequeno, pela fórmula de Taylor,

$$
v=1+\frac{\lambda}{2}+o(\lambda)
$$

Também, pela fórmula de Taylor, para $\lambda$ grande $v=\lambda+\frac{1}{\lambda}+o\left(\frac{1}{\lambda}\right)$.

Na Tabela 1.4 apresentamos valores estimados de $v$, juntamente com os obtidos por (1.5.11) 


\begin{tabular}{|c|c|c|c|c|c|}
\hline$\lambda$ & $\mathrm{T}$ & $\mathrm{N}$ & valor teórico & valor estimado & diferença relativa \\
\hline 0.1 & 2000 & 200 & 1.0512 & 1.0429 & 0.0079 \\
0.3 & 2000 & 100 & 1.1612 & 1.1731 & 0.0102 \\
0.5 & 2000 & 100 & 1.2808 & 1.3123 & 0.0246 \\
1 & 2000 & 50 & 1.6180 & 1.6343 & 0.0100 \\
1.5 & 2000 & 50 & 2 & 1.9593 & 0.0203 \\
2 & 2000 & 50 & 2.4142 & 2.3844 & 0.0123 \\
2.5 & 1000 & 50 & 2.8508 & 2.8873 & 0.0128 \\
3 & 1000 & 50 & 3.3028 & 3.2043 & 0.0298 \\
4 & 1000 & 40 & 4.2360 & 4.1361 & 0.0236 \\
5 & 1000 & 40 & 5.1926 & 5.1108 & 0.0157 \\
\hline
\end{tabular}

Tabela 1.4: Valores teóricos e estimados para $v$

No que segue mostraremos que para $f(\eta)=I_{\{\eta(1)=0\}}$ a equação (1.5.1) não é satisfeita. Queremos obter uma expressão para $G f(\eta)$. Para $x \leq-1$ ou $x \geq 2$,

$$
\eta(x)\left(f\left(\eta^{x, x+1}\right)-f(\eta)\right)=\eta(x) 0=0 .
$$

Quando $x=1$ poderá acontecer os seguintes casos:

$$
\begin{aligned}
& \eta(1)=0: \eta(1)\left(f\left(\eta^{1,2}\right)-f(\eta)\right)=0 \\
& \eta(1)=1: \\
& \eta(1)\left(f\left(\eta^{1,2}\right)-f(\eta)\right)=\eta(1)\left(I_{\{\eta(1)-1=0\}}-I_{\{\eta(1)=0\}}\right)=I_{\{\eta(1)=1\}}
\end{aligned}
$$

$\eta(1)>1:$

$$
\eta(1)\left(f\left(\eta^{1,2}\right)-f(\eta)\right)=\eta(1)\left(I_{\{\eta(1)-1=0\}}-I_{\{\eta(1)=0\}}\right)=\eta(1)(0-0)=0 .
$$

Com relação ao sítio zero, 


$$
(\eta(0)+1)(f(\overleftarrow{\eta})-f(\eta))=(\eta(0)+1)\left(I_{\{\eta(2)=0\}}-I_{\{\eta(1)=0\}}\right)
$$

Os casos em que esta parcela não se anula são

$$
\{\eta(1)>0 \cap \eta(2)=0\} \quad \text { ou } \quad\{\eta(1)=0 \cap \eta(2)>0\}
$$

Então,

$$
(\eta(0)+1)(f(\overleftarrow{\eta})-f(\eta))=(\eta(0)+1)\left(I_{\{\eta(1)>0 \cap \eta(2)=0\}}-I_{\{\eta(1)=0 \cap \eta(2)>0\}}\right)
$$

e portanto,

$$
G f(\eta)=I_{\{\eta(1)=1\}}+(\eta(0)+1)\left(I_{\{\eta(1)>0 \cap \eta(2)=0\}}-I_{\{\eta(1)=0 \cap \eta(2)>0\}}\right) .
$$

Aplicando esperaça com relação à medida-produto $\mu$ em (1.5.13), tem-se

$$
\begin{aligned}
E_{\mu} G f(\eta) & =P(\eta(1)=1)+\left(E_{\mu} \eta(0)+1\right) \times \\
& \times[P(\eta(1)>0) P(\eta(2)=0) \\
& -P(\eta(1)=0) P(\eta(2)>0)] .
\end{aligned}
$$

Em (1.5.14), com a suposição de que $\eta(i) \sim \operatorname{Pois}(\nu(i)), i \in\{0,1,2\}$ e $\nu(i)$ dado por (1.5.8), teremos

$$
\begin{aligned}
E_{\mu} G f(\eta) & =\nu(1) e^{-\nu(1)}+(a+1)\left[\left(1-e^{-\nu(1)}\right) e^{-\nu(2)}-e^{-\nu(1)}\left(1-e^{-\nu(2)}\right)\right] \\
& =\nu(1) e^{-\nu(1)}+(a+1)\left[e^{-\nu(2)}-e^{-\nu(1)}\right]
\end{aligned}
$$

Mas como $\nu(1)$ e $\nu(2)$ são funções de $\lambda$ e $a$, e por sua vez $a$ é dada por (1.5.10), então (1.5.15) será função apenas de $\lambda$, ou seja, $E_{\mu} G f(\eta)=h(\lambda)$. A função $h$ é positiva para 


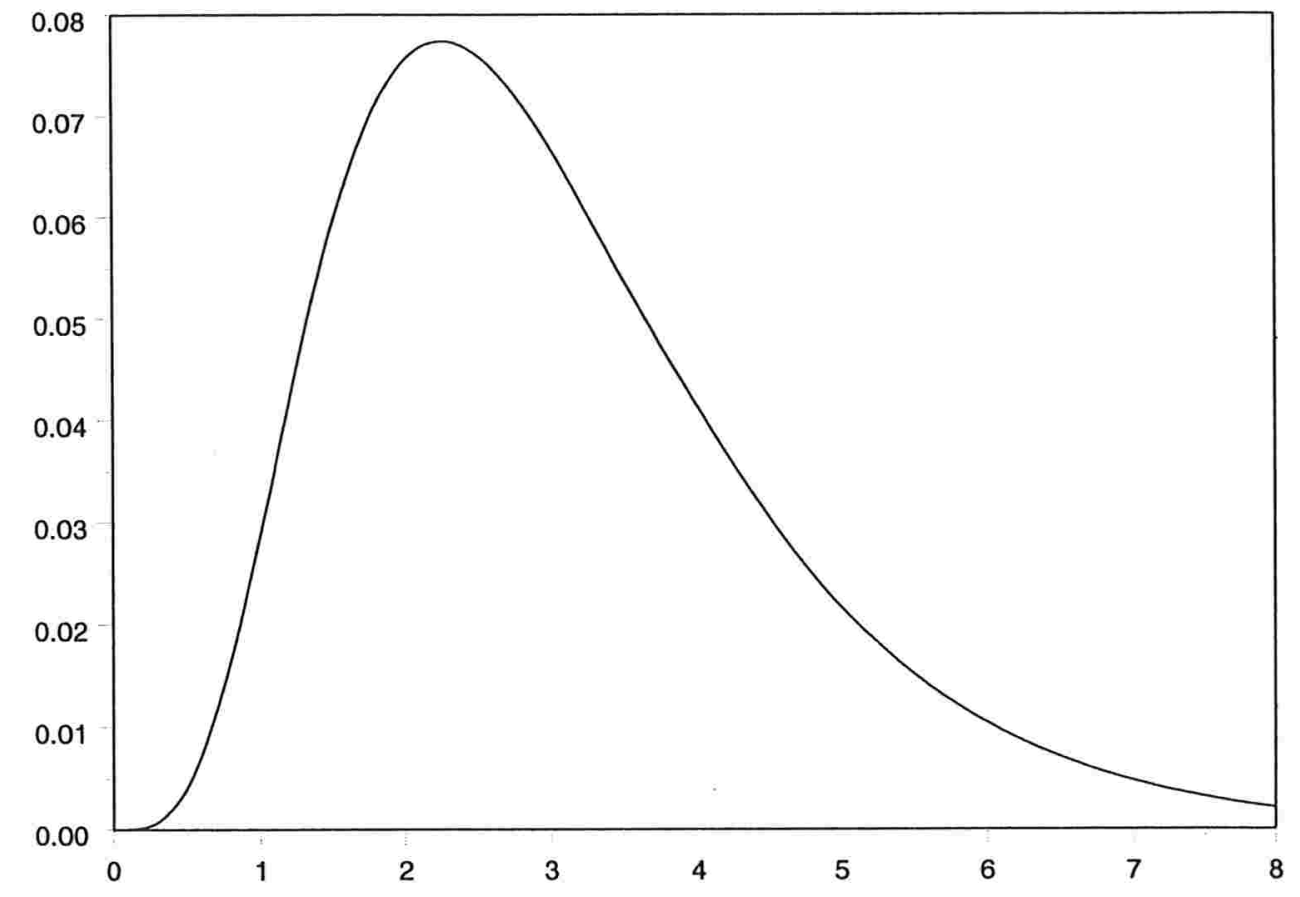

Figura 1.3: Gráfico de $\operatorname{EGf}(\eta)$ em função de $\lambda$ quando $f(\eta)=I_{\{\eta(1)=0\}}$. 
todo $\lambda>0$, conforme vemos na Figura 1.3 (obtida pelo programa Scilab). Isto nos permite concluir que $E_{\mu} G f(\eta)>0$, e assim, $f(\eta)=I_{\{\eta(1)=0\}}$ não satisfaz (1.5.1).

Em (1.5.9) fizemos a suposição de que $\eta(i) \sim \operatorname{Pois}(\nu(i))$, sendo $\nu(i)$ dado por (1.5.8). Façamos uma comparação entre as distribuições teóricas e as freqüências relativas obtidas nas simulações. Para isto, defina $p_{i}(j)=P(\eta(i)=j)$ e $f_{i}(j)$ como sendo a freqüência relativa do evento $\{\eta(i)=j\}$, para $j=0,1, \ldots, 6$. As freqüências relativas são obtidas através do número de iterações, que corresponde ao número de repetições do experimento.

Executamos o programa para $\lambda \in\{1,2\}, \mathrm{N}=50, \mathrm{n}=10$ e $\mathrm{T}=1000$. O teste quiquadrado de ajustamento à distribuição de Poisson foi também realizado. Observando as Tabelas 1.5 e 1.6 vemos que as maiores discrepâncias entre probabilidades e freqüências relativas estão no sítio zero. Por exemplo, para $\lambda=2, p_{0}(0)=0.2431 \mathrm{e}$ $f_{0}(0)=0.2773$. Para sítios negativos a concordância é boa. Concluimos que a medida invariante deve ser próxima à medida-produto, pelo menos para sítios abaixo do zero. Outra comparação é entre as freqüências relativas conjuntas e o produto das marginais. Para tal utilizamos o teste qui-quadrado de independência para alguns pares de sítios. Simulamos para $\lambda \in\{1,2\}, \mathrm{N}=50$ e $\mathrm{T}=1000$. Na Tabela 1.7 encontram-se os resultados. Vemos que os sítios 1 e 2 não apresentam independência quanto ao número de partículas, contudo, parece que existe independência nos outros pares de sítios. 


\begin{tabular}{|c|c|c|c|c|c|c|c|c|}
\hline sítio & $p_{i}(j)$ & $f_{i}(j)$ & sítio & $p_{i}(j)$ & $f_{i}(j)$ & sítio & $p_{i}(j)$ & $f_{i}(j)$ \\
\hline-10 & 0.3678 & 0.3472 & -1 & 0.3678 & 0.3482 & 3 & 0.4658 & 0.5010 \\
$(0.0544)$ & 0.3678 & 0.3919 & $(0.3037)$ & 0.3678 & 0.3660 & $(0.2571)$ & 0.3558 & 0.3363 \\
& 0.1839 & 0.1915 & & 0.1839 & 0.2093 & & 0.1359 & 0.1220 \\
& 0.0613 & 0.0446 & & 0.0613 & 0.0575 & & 0.0346 & 0.0337 \\
& 0.0153 & 0.0158 & & 0.0153 & 0.0188 & & 0.0066 & 0.0059 \\
& 0.0031 & 0.0060 & & 0.0031 & 0.0000 & & 0.0010 & 0.0000 \\
& 0.0005 & 0.0020 & & 0.0005 & 0.0000 & & 0.0001 & 0.0010 \\
\hline-5 & 0.3678 & 0.3452 & 0 & 0.5390 & 0.5665 & 5 & 0.4026 & 0.4534 \\
$(0.0953)$ & 0.3678 & 0.3562 & $(0.0008)$ & 0.3331 & 0.2976 & $(0.0177)$ & 0.3663 & 0.3383 \\
& 0.1839 & 0.2153 & & 0.1029 & 0.1062 & & 0.1666 & 0.1528 \\
& 0.0613 & 0.0605 & & 0.0212 & 0.0188 & & 0.0505 & 0.0466 \\
& 0.0153 & 0.0198 & & 0.0033 & 0.0060 & & 0.0115 & 0.0069 \\
& 0.0031 & 0.0030 & & 0.0004 & 0.0030 & & 0.0021 & 0.0020 \\
& 0.0005 & 0.0000 & & 0.0000 & 0.0000 & & 0.0003 & 0.0000 \\
\hline \multirow{2}{*}{$(0.4297)$} & 0.3678 & 0.3442 & 1 & 0.6825 & 0.7163 & 10 & 0.3709 & 0.3532 \\
& 0.1839 & 0.1875 & & 0.0497 & 0.0476 & & 0.1824 & 0.1746 \\
& 0.0613 & 0.0615 & & 0.0063 & 0.0069 & & 0.0603 & 0.0600 \\
& 0.0153 & 0.0218 & & 0.0006 & 0.0010 & & 0.0149 & 0.0178 \\
& 0.0005 & 0.0010 & & 0.0000 & 0.0000 & & 0.0005 & 0.0020 \\
\hline
\end{tabular}

Tabela 1.5: Comparação entre $p_{i}(j)$ e $f_{i}(j)$ para $\lambda=1$. Valores entre parênteses são os níveis descritivos para o teste qui-quadrado de ajustamento 


\begin{tabular}{|c|c|c|c|c|c|c|c|c|}
\hline sítio & $p_{i}(j)$ & $f_{i}(j)$ & sítio & $p_{i}(j)$ & $f_{i}(j)$ & sítio & $p_{i}(j)$ & $f_{i}(j)$ \\
\hline-10 & 0.1353 & 0.1273 & -1 & 0.1353 & 0.1067 & 3 & 0.1560 & 0.1595 \\
\hline \multirow[t]{6}{*}{$(0.4204)$} & 0.2706 & 0.2677 & $(0.0103)$ & 0.2706 & 0.2788 & $(0.5218)$ & 0.2898 & 0.2889 \\
\hline & 0.2706 & 0.2723 & & 0.2706 & 0.2843 & & 0.2692 & 0.2763 \\
\hline & 0.1804 & 0.1766 & & 0.1804 & 0.1902 & & 0.1667 & 0.1686 \\
\hline & 0.0902 & 0.1046 & & 0.0902 & 0.0911 & & 0.0774 & 0.0730 \\
\hline & 0.0361 & 0.0342 & & 0.0361 & 0.0322 & & 0.0287 & 0.0211 \\
\hline & 0.0120 & 0.0121 & & 0.0120 & 0.0116 & & 0.0089 & 0.0091 \\
\hline-5 & 0.1353 & 0.1454 & 0 & 0.2431 & 0.2773 & 5 & 0.1386 & 0.1359 \\
\hline \multirow[t]{6}{*}{$(0.3158)$} & 0.2706 & 0.2607 & $(0.0143)$ & 0.3438 & 0.3246 & $(0.4061)$ & 0.2740 & 0.2617 \\
\hline & 0.2706 & 0.2652 & & 0.2431 & 0.2396 & & 0.2706 & 0.2859 \\
\hline & 0.1804 & 0.1787 & & 0.1146 & 0.1122 & & 0.1782 & 0.1892 \\
\hline & 0.0902 & 0.1022 & & 0.0405 & 0.0322 & & 0.0880 & 0.0080 \\
\hline & 0.0361 & 0.0327 & & 0.0114 & 0.0101 & & 0.0348 & 0.0322 \\
\hline & 0.0120 & 0.0131 & & 0.0027 & 0.0025 & & 0.0114 & 0.0136 \\
\hline-3 & 0.1353 & 0.1339 & 1 & 0.3098 & 0.3236 & 10 & 0.1354 & 0.1404 \\
\hline \multirow[t]{6}{*}{$(0.1768)$} & 0.2706 & 0.2713 & $(0.1345)$ & 0.3630 & 0.3417 & $(0.3922)$ & 0.2707 & 0.2587 \\
\hline & 0.2706 & 0.2612 & & 0.2126 & 0.2159 & & 0.2706 & 0.2889 \\
\hline & 0.1804 & 0.1857 & & 0.0831 & 0.0856 & & 0.1804 & 0.1701 \\
\hline & 0.0902 & 0.0820 & & 0.0243 & 0.0221 & & 0.0902 & 0.0870 \\
\hline & 0.0361 & 0.0458 & & 0.0057 & 0.0091 & & 0.0361 & 0.0398 \\
\hline & 0.0120 & 0.0141 & & 0.0011 & 0.0010 & & 0.0120 & 0.0116 \\
\hline
\end{tabular}

Tabela 1.6: Comparação entre $p_{i}(j)$ e $f_{i}(j)$ para $\lambda=2$. Valores entre parênteses são os níveis descritivos para o teste qui-quadrado de ajustamento 


\begin{tabular}{|c|c|c|}
\hline sítios & $\lambda=1$ & $\lambda=2$ \\
\hline$(-2,-1)$ & 0.3262 & 0.5442 \\
$(-1,1)$ & 0.3253 & 0.1417 \\
$(0,1)$ & 0.1352 & 0.4681 \\
$(1,2)$ & 0.0007 & 0.0137 \\
\hline
\end{tabular}

Tabela 1.7: Níveis descritivos para o teste qui-quadrado de independência

\subsection{Uma medida dominante para o modelo cen- trado}

Já vimos anteriormente que a medida-produto não é a medida invariante para o processo. Na seção anterior apresentamos um exemplo que não satisfaz a equação (1.5.1). Contudo, pelos resultados das simulações podemos suspeitar que a medida invariante do processo é próxima à medida-produto. O lema a seguir (Lema 2.7 extraído de [16]) mostra que a medida do processo em relação aos sítios $\{1,2, \ldots\}$ é dominada por uma medida-produto. Aqui enunciamos o lema para o modelo específico tratado neste capítulo.

Lema 1.6.1. Assuma que a configuração inicial segue a medida-produto $\mathbb{B}_{q}=\bigotimes_{x \in \mathbb{Z}}$ Pois $(q(x))$. Sejam $\mathcal{F}_{t}$ a $\sigma$-álgebra gerada pelo processo de propagação da infecção até o instante $t$ e $\gamma_{t}(x)$ o número de partículas normais no sítio $x \geq 1$. Então, a cada instante $t,\left\{\gamma_{t}(x)\right\}_{x \geq 1}$ é dominado pelo processo $\left\{\hat{\gamma}_{t}(x)\right\}_{x \geq 1}$, o qual é independente de $\mathcal{F}_{t}$ e tem lei de probabilidade $\mathbb{B}_{q_{t}}=\otimes_{x \geq 1} \operatorname{Pois}\left(q_{t}(x)\right)$, sendo $q_{t}(x)=\sum_{y} p_{t}(y, x)$ e $p_{t}(y, x)$ a probabilidade de alcançar o sítio $x$, partindo de $y$.

Prova: a demonstração completa está em [16], Lema 2.7. Contudo, faremos um esboço adaptado ao processo aqui estudado. Uma vez que começamos com partículas normais em $\{1,2, \ldots\}$, a medida dominante que obteremos será para sítios positivos. Como 
já sabemos, no processo aqui tratado a configuração inicial é a medida-produto de $\operatorname{Pois}(\lambda)$. Assim, $\mathbb{B}_{q}=\otimes_{x \in \mathbb{Z}}$ Pois $(\lambda)$. Vamos denotar por $X^{y}(t)$ a posição da partícula no instante $t$, quando esta partiu do sítio $y$ e iniciou um deslocamento à direita em $\mathbb{Z}$ com taxa 1 (note que em [16] as partículas realizam um passeio aleatório simples de taxa 1 em $\mathbb{Z}^{d}$ ).

Mas,

$$
p_{t}(y, x)=P\left(X^{y}(t)=x\right)=P\left(X^{0}(t)=x-y\right)=e^{-t} \frac{t^{x-y}}{(x-y) !},
$$

onde a última igualdade é justificada pelo fato de que o número de saltos é Pois $(t)$. Disso decorre que

$$
q_{t}(x)=\sum_{y \leq x} p_{t}(y, x) q(y)=\lambda \sum_{y \leq x} e^{-t} \frac{t^{x-y}}{(x-y) !}=\lambda .
$$

Isto quer dizer que começando com a medida-produto de Pois $(\lambda)$ o processo será dominado por um outro com lei $\mathbb{B}_{q_{t}}=\otimes_{x \geq 1} \operatorname{Pois}(\lambda)$.

Defina $\mathcal{M}$ como sendo o conjunto de todas as trajetórias finitas até o instante $t$. Um elemento $\omega \in \mathcal{M}$ é tal que $\omega=\left(x_{1}, \ldots, x_{n} ; t_{1}, \ldots, t_{n-1}\right)$, com $x_{i} \in \mathbb{Z},\left|x_{i}-x_{i+1}\right|=1$ e $0<t_{1}<t_{2}<\cdots<t_{n-1} \leq t$. O instante $t_{i}$ é o momento de salto de $x_{i}$ para $x_{i+1}$. A trajetória $\omega$ é tal que $|\omega|=n$, ou seja, $|\omega|$ é o número de saltos que ocorreram. Para $0 \leq s \leq t$, seja $\omega(s)$ a posição da trajetória no instante $s$, isto é,

$$
\omega(s)=\left\{\begin{array}{ll}
x_{1}, & \text { se } s<t_{1} \\
x_{i}, & \text { se } t_{i-1} \leq s<t_{i} \\
x_{n}, & \text { se } t_{n-1} \leq s
\end{array} .\right.
$$

Também precisamos definir 


$$
\begin{aligned}
\mathcal{M}_{x, n} & :=\{\omega \in \mathcal{M}: \omega(0)=x,|\omega|=n\}=\Psi_{x, n} \otimes \Phi_{n} \\
\Psi_{x, n} & :=\text { "conjunto das trajetórias com }|\omega|=n \text { começando em } x " \\
\Phi_{n} & :=\left\{\left(t_{1}, \ldots, t_{n-1}\right): 0<t_{1}, \ldots<t_{n-1} \leq t\right\}, n>1 \\
\Phi_{1} & :=\emptyset .
\end{aligned}
$$

Observe que partindo de $x_{1}$ há apenas uma trajetória com $|\omega|=n$, pois o processo se desloca apenas à direita e em dimensão 1 . Logo, $\Psi_{x, n}$ é unitário. Prosseguindo, $\mathcal{M}=$ $\bigcup_{x, n} \mathcal{M}_{x, n}$. Uma medida $\Lambda$ em $\mathcal{M}$ é tal que para $A \in \mathcal{M}, \Lambda(A)=\sum_{x, n}\left(A \cap \mathcal{M}_{x, n}\right)$. Assim, $\Lambda$ é definida para $A=\nu \times A_{0}, \nu \subset \Psi_{x, n}$ e $A_{0} \subset \Phi_{n}$, da seguinte maneira:

$$
\Lambda\left(\nu \times A_{0}\right):=\left\{\begin{array}{ll}
q(x) e^{-t}\left|A_{0}\right| & , n>1 \\
q(x) e^{-t} & , n=1
\end{array} .\right.
$$

Agora defina $\mathcal{N}$ como o conjunto aleatório dos pontos de Poisson que caem dentro de $A \in \mathcal{M}$ e $N(A)$ sua cardinalidade. Através de um algoritmo similar ao método da geração, usado em percolação, contrói-se o conjunto $\mathcal{B}$ de forma que $\mathcal{N}(\mathcal{B})$ é o conjunto das trajetórias infectadas até o momento $t$, e além disso, $\mathcal{B}$ é independente de $\mathcal{N}(\mathcal{M} \mid \mathcal{B})$.

Seja $\mathcal{N}^{\prime}(\mathcal{M})$ outro processo de Poisson com medida $\Lambda$, independente de $\mathcal{N}(\mathcal{M})$. Como antes, $N^{\prime}(\cdot)$ é a cardinalidade de $\mathcal{N}^{\prime}(\cdot)$. Também definimos

$$
\hat{\gamma}_{t}(x)=N\left((\{\omega: \omega(t)=x, \omega \notin \mathcal{B}\})+N^{\prime}((\{\omega: \omega(t)=x, \omega \in \mathcal{B}\}) .\right.
$$

Uma vez que $\gamma_{t}(x)=N\left((\{\omega: \omega(t)=x, \omega \notin \mathcal{B}\})\right.$ então $\left\{\gamma_{t}(x)\right\}_{x \geq 1}$ é dominado por $\left\{\hat{\gamma}_{t}(x)\right\}_{x \geq 1}$. Para completar,

$$
\begin{aligned}
E \hat{\gamma}_{t}(x) & =E_{\Lambda}\left(N((\{\omega: \omega(t)=x, \omega \notin \mathcal{B}\}))+E_{\Lambda}\left(N^{\prime}((\{\omega: \omega(t)=x, \omega \in \mathcal{B}\}))\right.\right. \\
& =E_{\Lambda}\left(\sum_{\omega} I_{\{\omega: \omega(t)=x\}}\right)=\Lambda(\{\omega: \omega(t)=x\})=\sum_{y} q(y) p_{t}(y, x) .
\end{aligned}
$$




\subsection{Conexão com o Movimento Browniano}

Na seção 1.1 definimos o modelo de maneira que a distância entre dois sítios vizinhos era 1 e cada partícula tinha associado um Processo de Poisson de taxa 1. Desta forma, a posição $X_{t}$ em $\mathbb{Z}$ de uma partícula é Pois $(t)$. Agora vamos admitir que a distância entre dois sítios vizinhos seja $0<\lambda<1$. Se fizermos a transformação $\lambda X_{\lambda^{-2}}$, então mudaremos para o espaço de estados $\lambda \mathbb{Z}$ e para cada partícula estará associado um Processo de Poisson de taxa $\lambda^{-2}$. Por isso, os instantes de saltos formarão uma seqüência de v.a's iid $\operatorname{Expon}\left(\lambda^{-2}\right)$. Seja

$$
Y_{t}:=\lambda\left(X_{\lambda^{-2} t}-\lambda^{-2} t\right)=\lambda X_{\lambda^{-2} t}-\lambda^{-1} t
$$

Logo,

$$
\begin{aligned}
E\left(Y_{t}\right) & =\lambda E\left(X_{\lambda^{-2} t}\right)-\lambda^{-1} t=0 \\
\operatorname{Var}\left(Y_{t}\right) & =\lambda^{2} \operatorname{Var}\left(X_{\lambda^{-2} t}\right)=t .
\end{aligned}
$$

A equação (1.7.2) significa que o deslocamento médio de $Y_{t}$ será zero. Podemos ainda dizer que a taxa de deslocamento à direita será $\lambda^{-2}$, mas à esquerda haverá um deslocamento constante de taxa $\lambda^{-1}$, e na média o deslocamento será nulo.

A seqüência $\left\{Y_{t}\right\}_{t \geq 0}$ tem incrementos independentes, decorrente do fato de que os instantes de saltos são iid. Além disso, como observamos em (1.7.2) e (1.7.3), tem média zero e variância igual ao comprimento do intervalo. Para justificar a aproximação ao Movimento Browniano falta ainda exprimir $Y_{t}$ como uma soma parcial de v.a's iid. Com $t>0$ e $\lambda>0$ fixados ocorrerá aproximadamente $n=\left\lfloor\lambda^{-2} t\right\rfloor$ deslocamentos. Sendo assim, poderemos aproximar $Y_{t}$ como uma soma finita, ou seja,

$$
Y_{t} \approx Y_{n}=\lambda\left(X_{n}-n\right)=\sum_{i=1}^{n} \lambda\left(V_{i}-1\right)
$$


onde $\left\{V_{i}\right\}_{i \geq 1}$ são v.a's iid com $V_{i} \sim \operatorname{Pois}(1)$, pois $X_{n} \sim \operatorname{Pois}(n)$. Denotando $W_{i}:=$ $\lambda\left(V_{i}-1\right)$ vemos que $Y_{n}$ é uma soma de v.a's iid com $E\left(W_{i}\right)=0$ e $\operatorname{Var}\left(W_{i}\right)=\lambda^{2}$. Note que $\left\{Y_{n}\right\}_{n \geq 1}$ é um processo a tempo discreto e $\left\{Y_{t}\right\}_{t \geq 0}$ contínuo. No caso em que $\lambda^{-2} t=n$ ambos processos coincidem. Para $\lambda^{-2} t$ real iremos comparar $Y_{t}$ com o processo obtido por interpolação linear, definido como

$$
Y_{t}^{(\lambda)}:=Y_{\left\lfloor\lambda^{-2} t\right\rfloor}+\left(\lambda^{-2} t-\left\lfloor\lambda^{-2} t\right\rfloor\right) W_{\left\lfloor\lambda^{-2} t\right\rfloor+1}=Y_{n}+\left(\lambda^{-2} t-n\right) W_{n+1}
$$

Decorre que

$$
\begin{aligned}
\left|Y_{t}-Y_{t}^{(\lambda)}\right| & =\left|\lambda\left(X_{\lambda^{-2} t}-\lambda^{-2} t\right)-\sum_{i=1}^{n} W_{i}-\left(\lambda^{-2} t-n\right) W_{n+1}\right| \\
& =\lambda\left|X_{\lambda^{-2} t}-\sum_{i=1}^{n} V_{i}-\left(\lambda^{-2} t-n\right) V_{n+1}\right| \\
& \leq \lambda\left(\left|X_{\lambda^{-2} t}-\sum_{i=1}^{n} V_{i}\right|+\left|\lambda^{-2} t-n\right|\left|V_{n+1}\right|\right) \\
& \leq \lambda\left(\left|X_{\lambda^{-2} t}-\sum_{i=1}^{n} V_{i}\right|+\left|V_{n+1}\right|\right)
\end{aligned}
$$

Seja $\epsilon>0$. Por (1.7.5),

$$
\begin{aligned}
P\left(\left|Y_{t}-Y_{t}^{(\lambda)}\right|>\epsilon\right) & \leq P\left(\left|X_{\lambda^{-2} t}-\sum_{i=1}^{n} V_{i}\right|+\left|V_{n+1}\right|>\frac{\epsilon}{\lambda}\right) \\
& \leq \frac{\left(\lambda^{-2} t-n+1\right) \lambda}{\epsilon} .
\end{aligned}
$$

A última desigualdade em (1.7.6) é justificada pela desigualdade básica de Markov e também por $\left(X_{\lambda^{-2} t}-\sum_{i=1}^{n} V_{i}\right) \sim \operatorname{Pois}\left(\lambda^{-2} t-n\right), V_{n+1} \sim \operatorname{Pois}(1)$. Como

$$
\lim _{\lambda ! 0} \frac{\left(\lambda^{-2} t-n+1\right) \lambda}{\epsilon}=0
$$


$Y_{t}^{(\lambda)}$ converge em probabilidade para $Y_{t}$. Na Figura 1.4 vemos que à medida que $\lambda \downarrow 0$ podemos colocar a distância $\left|Y_{t}-Y_{t}^{(\lambda)}\right|$ dentro de um trapézio limitado.

Pelo Teorema que segue iremos argumentar que $\left\{Y_{t}\right\}_{t \geq 0}$ se aproxima de $\left\{B_{t}\right\}_{t \geq 0}$, Movimento Browniano padrão, no sentido forte (quase certamente).

Teorema 1.7.1. (Komlós, Major, Tusnády) Seja $\left\{\xi_{i}\right\}_{i \geq 1}$ seqüência de v.a's iid tais que $E \xi_{i}=0$ e $\operatorname{Var}\left(\xi_{i}\right)=1$. Também assuma que $E\left(\exp \left\{t \xi_{i}\right\}\right)$ exista em uma vizinhança de $t=0$. Seja $S_{n}:=\sum_{i=1}^{n} \xi_{i}$. Então, existe um Movimento Browniano padrão $\left\{B_{t}\right\}_{t \geq 0}$ tal que existe $K>0$ satisfazendo

$$
P\left(\frac{\left|S_{n}-B_{n}\right|}{\ln n} \leq K, \text { para quase todo } n\right)=1 .
$$

Prova: [5], Teorema 2.6.1.

O Teorema 1.7.1 diz que existe um acoplamento entre o processo das somas parciais e o Movimento Browniano. Ainda, de (1.7.7), decorre que existe $C>0$ finito tal que

$$
P\left(\limsup _{n \rightarrow \infty} \frac{\left|S_{n}-B_{n}\right|}{O(\ln n)} \leq C\right)=1
$$

As condições do Teorema 1.7.1 são satisfeitas para $\left\{Y_{t}^{(\lambda)}: \lambda>0, t \geq 0\right\}$, pois $E\left(V_{i}-1\right)=0, \operatorname{Var}\left(V_{i}-1\right)=1, E\left(\exp \left\{t\left(V_{i}-1\right)\right\}\right)=\exp \left\{e^{-t}-t-1\right\}<\infty$, para qualquer $t$ real, e também $Y_{t}^{(\lambda)}$ é expressa como uma soma parcial. Logo, temos uma aproximação forte de $\left\{Y_{t}^{(\lambda)}: \lambda>0, t \geq 0\right\}$ a $\left\{B_{t}\right\}_{t \geq 0}$, quando $\lambda \downarrow 0$. Por outro lado, como podemos ver por (1.7.5), as trajetórias de $\left\{Y_{t}\right\}_{t \geq 0}$ e $\left\{Y_{t}^{(\lambda)}: \lambda>0, t \geq 0\right\}$ não diferem significativamente para $\lambda$ pequeno, e portanto, também teremos uma aproximação forte de $\left\{Y_{t}\right\}_{t \geq 0}$ a $\left\{B_{t}\right\}_{t \geq 0}$.

Ainda com relação ao processo $\left\{Y_{t}\right\}_{t \geq 0}$, definido em (1.7.1), vamos admitir que a configuração inicial seja a medida-produto de $\operatorname{Pois}(\lambda)$. Desta forma, teremos o seguinte teorema: 


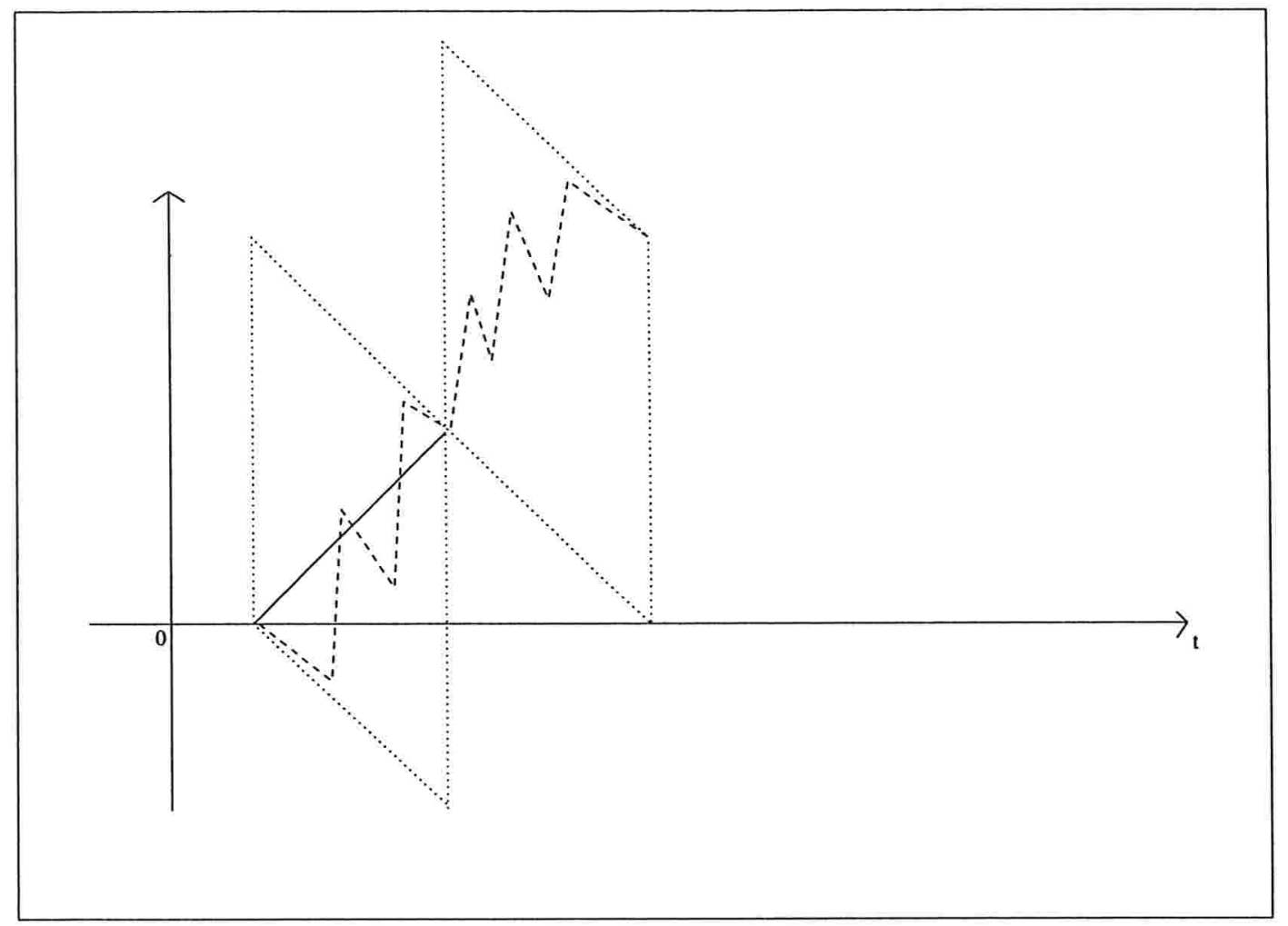

Figura 1.4: Comparação gráfica entre $Y_{t}^{\lambda}$ e $Y_{t}$. Linha tracejada é $Y_{t}$ e a cheia é $Y_{t}^{\lambda}$ 
Teorema 1.7.2. Seja o processo definido por (1.7.1). Com a medida-produto de $\operatorname{Pois}(\lambda)$ como configuração inicial, tomando $\lambda \downarrow 0$, teremos que esta converge para o Processo de Poisson Homogêneo de taxa 1.

Prova: para $\lambda \in(0,1)$ fixado, o conjunto de sítios é $\{\ldots,-2 \lambda,-\lambda, 0, \lambda, 2 \lambda, \ldots\}$. Fazendo $\lambda \downarrow 0$ teremos este conjunto convergindo para $\mathbb{R}$. Vamos denotar por $\{N(x, y)$ : $-\infty<x<y<\infty\}$ o processo de contagem do número de partículas no intervalo $(x, y]$. Precisamos verificar os três requisitos para que seja um Processo de Poisson:

(1o) incrementos independentes;

(20) incrementos estacionários;

(3o)

$$
P(N(x, x+h)=m)=\left\{\begin{array}{ll}
1-h+o(h), & \text { se } m=0 \\
h+o(h), & \text { se } m=1 \\
\circ(h), & \text { se } m>1
\end{array} .\right.
$$

Os itens (1o) e (2o) são imediatos, pois decorrem do fato da configuração inicial ser a medida-produto de $\operatorname{Pois}(\lambda)$. Quanto ao (3o) $)$, no intervalo $(x, x+h]$ haverá aproximadamente $\frac{h}{\lambda}$ pontos, e portanto, pela independência da configuração inicial,

$$
P(N(x, x+h)=0)=\left(e^{-\lambda}\right)^{\frac{h}{\lambda}}=e^{-h}=1-h+\mathrm{o}(h) .
$$

Também teremos que

$$
P(N(x, x+h)=1)=\frac{h}{\lambda} \lambda e^{-\lambda}\left(e^{-\lambda}\right)^{\frac{h}{\lambda}-1}=h e^{-h}=h+\bar{o}(h)
$$

e $P(N(x, x+h)>1)=\tilde{\mathrm{o}}(h)$. Logo, concluimos que se trata de um Processo de Poisson de taxa 1 . 
Na seção 1.1, por (1.1.8), mostramos que a velocidade da partícula marcada é $v_{m}=1$, quase certamente. Com este fato, juntamente com (1.5.12), quando a distância entre sítios é $\lambda \in(0,1)$ e a taxa de deslocamento à direita é $\lambda^{-2}$ podemos conjecturar que a velocidade da partícula marcada no processo será

$$
\frac{\lambda}{\lambda^{2}}\left(v-v_{m}\right)=\frac{1}{\lambda}\left(\frac{\lambda}{2}+o(\lambda)\right)=\frac{1}{2}+\frac{o(\lambda)}{\lambda} .
$$

$\operatorname{Em}(1.7 .8), \lim _{\lambda \downarrow 0}\left(\frac{1}{2}+\frac{\mathrm{o}(\lambda)}{\lambda}\right)=\frac{1}{2}$. Note que este limite é uma conjectura, visto que (1.5.12) foi obtido com base nos resultados empíricos das simulações. Isto significa que quando $\lambda \downarrow 0$ podemos esperar que a trajetória de cada partícula marcada convirja para o Movimento Browniano. No Capítulo 2 faremos simulações para reforçar esta conjectura.

\subsection{Conclusões}

Neste capítulo analisamos a velocidade de contaminação através de simulações. Por meio de um contra-exemplo verificamos que a medida-produto não é a medida invariante para o modelo centrado. Contudo, as simulações mostraram que as estimativas de velocidade ficaram próximas da velocidade teórica, obtida com a suposição de que a medida invariante é a produto de Poisson. Além disso, para sítios normais, ou seja, para frente da infecção, mostramos que existe uma medida dominante. Também verificamos que existe uma conexão com a velocidade em um Movimento Browniano padrão. 


\section{Capítulo 2}

\section{Propagação de infecção entre Movimentos Brownianos}

\section{independentes}

\subsection{Definindo o modelo}

No presente Capítulo o modelo considerado terá espaço de estados $\mathbb{R}$ e o tempo contínuo. A configuração inicial seguirá um Processo de Poisson Homogêneo de taxa 1 sobre $\mathbb{R}$, ou seja, o processo $\left\{N_{0}(x, y):-\infty<x<y<\infty\right\}, N_{0}(x, y)$ representando o número de partículas no intervalo $(x, y]$ no instante inicial, será tal que

$$
P\left(N_{0}(x, y)=k\right)=\frac{(y-x)^{k}}{k !} e^{-(y-x)}, \quad k=0,1, \ldots .
$$

Dado que $N_{0}(x, y)=k$, a posição da $j$-ésima partícula, $j=1, \ldots, k$, dentro do intervalo $(x, y]$ terá distribuição de probabilidade uniforme contínua. Também iremos assumir que na posição zero obrigatoriamente deverá haver uma partícula.

Após a alocação inicial de partículas em $\mathbb{R}$, segundo um Processo de Poisson, teremos uma quantidade infinita enumerável. Sendo assim, faremos uma bijeção entre as 
partículas atribuídas e o conjunto $\mathbb{Z}$. Mais precisamente, para a partícula que está na posição zero atribuimos o número zero, para partículas abaixo do zero atribuimos os inteiros negativos e aquelas acima do zero recebem os inteiros positivos.

A partir do instante inicial, cada partícula começará a se deslocar segundo um Movimento Browniano padrão, e estes serão mutuamente independentes. A posição da partícula $i, i \in \mathbb{Z}$, após decorrido um tempo $t$, será

$$
X_{t}(i)=X_{0}(i)+B_{t}(i)
$$

sendo $\left\{B_{t}(i)\right\}_{t \geq 0}$ o correspondente Movimento Browniano padrão associado à partícula, com $B_{0}(i)=0$. Assim,

$$
X_{0}(i)=\left\{\begin{array}{ll}
x<0, & \text { se } \quad i \in\{\ldots,-2,-1\} \\
0, & \text { se } i=0 \\
x>0, & \text { se } \quad i \in\{1,2, \ldots\}
\end{array} .\right.
$$

Ainda com relação a $\left\{B_{t}(i)\right\}_{t \geq 0}$, tem-se:

- Todo incremento $B_{t+s}(i)-B_{s}(i)$ tem distribuição normal de média zero e variância $t$.

- Para todo par de intervalos disjuntos de tempo $\left(t_{1}, t_{2}\right],\left(t_{3}, t_{4}\right]$, com $0 \leq t_{1}<t_{2} \leq$ $t_{3}<t_{4}$, os incrementos $B_{t_{4}}(i)-B_{t_{3}}(i)$ e $B_{t_{2}}(i)-B_{t_{1}}(i)$ são v.a's independentes. Similarmente vale para $n$ intervalos disjuntos.

- $B_{t}(i)$ é função contínua em relação à variável $t$.

Além da condições anteriormente feitas ao modelo, sem perda de generalidade assumiremos que no instante inicial partículas à esquerda do zero estarão contaminadas, e também a que está na posição zero. As partículas à direita do zero serão declaradas normais (saudáveis). Cada vez que uma partícula contaminada alcançar uma normal, então esta ficará contaminada. Estaremos interessados em estudar a propagação da 
infecção neste sistema, observando a velocidade de infecção $v:=\lim _{t \rightarrow \infty} \frac{Z_{t}}{t}, Z_{t}$ sendo a fronteira atual entre contaminados e normais. Na Figura 2.1 temos possíveis trajetórias de algumas partículas.

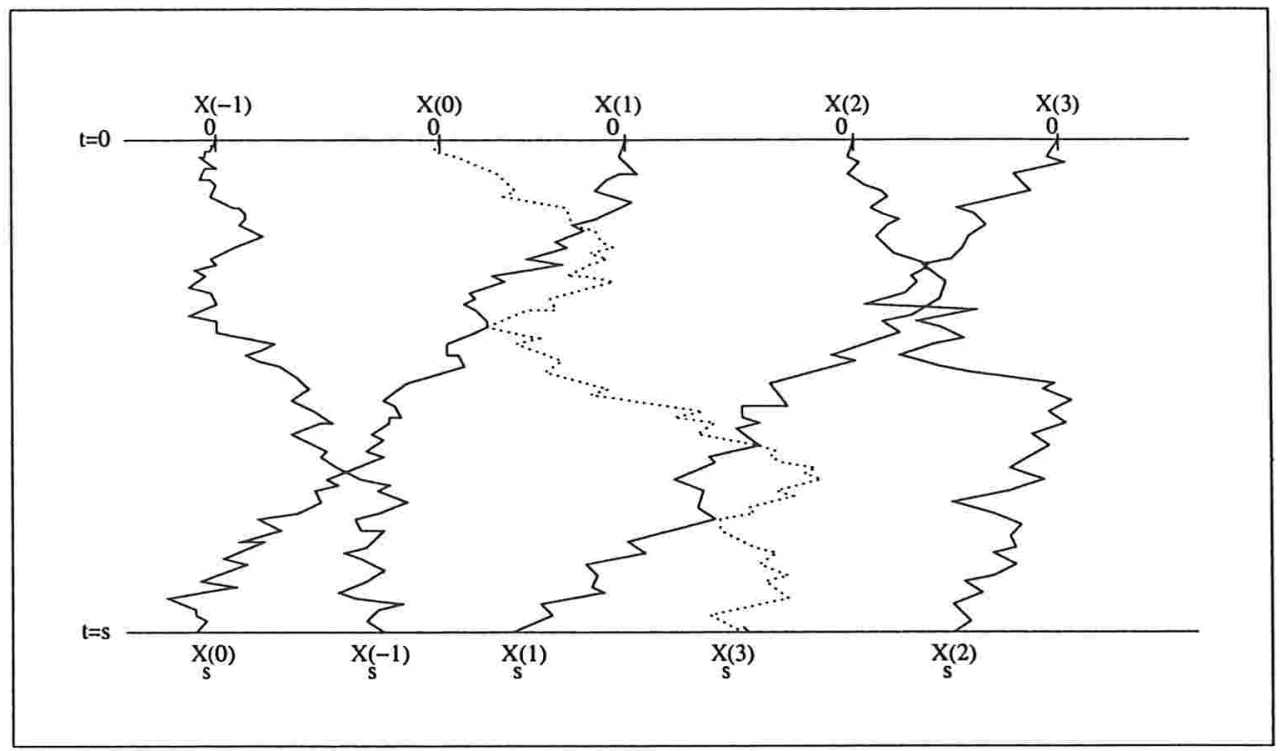

Figura 2.1: Possíveis trajetórias de algumas partículas que se deslocam segundo um Movimento Browniano padrão. A trajetória pontilhada se refere ao avanço da infecção.

A proposição a seguir será útil para mostrarmos que se no instante inicial forem atribuídas partículas em $\mathbb{R}$, segundo um Processo de Poisson Homogêneo de taxa 1, e estas começarem um deslocamento de acordo com um Movimento Browniano padrão, então, após decorrido um tempo $t$, também teremos um Processo de Poisson de taxa 1 em $\mathbb{R}$. Este resultado nos diz que, começando com um Processo de Poisson Homogêneo de taxa 1, ao longo do tempo ainda teremos um Processo de Poisson com esta mesma taxa. Isto significa que a medida invariante do modelo segue um Processo de Poisson Homogêneo de taxa 1. 
Proposição 2.1.1. Suponha que eventos ocorrem de acordo com um Processo de Poisson Homogêneo de taxa $\mu$. Além disso, independentemente do que aconteceu antes, suponha que um evento no instante $s$ é classificado como sendo do tipo $i$, com probabilidade $P_{s}(i), i=1, \ldots, k$. Se $N_{t}(i), t \geq 0$, denota o número de eventos do tipo $i$, então

$$
N_{t}(1), N_{t}(2), \ldots, N_{t}(k)
$$

são variáveis aleatórias independentes de média

$$
E\left(N_{t}(i)\right)=\mu \times \int_{0}^{t} P_{s}(i) d s
$$

Prova: esta proposição vem da Teoria das Filas, especificamente do modelo M/G/ $\infty$, e a prova está em [23], Proposição 5.3.

Teorema 2.1.1. Suponha que no instante inicial partículas sejam atribuídas em $\mathbb{R}$ de acordo com um Processo de Poisson Homogêneo de taxa 1. Também assuma que após o instante inicial essas partículas iniciam um deslocamento de acordo com um Movimento Browniano padrão. Então, $\left\{N_{t}(x, y):-\infty<x<y<\infty\right\}$, com $N_{t}(x, y)$ representando o número de partículas no intervalo $(x, y]$ após decorrido um tempo $t$, também será um Processo de Poisson de taxa 1.

Prova: a Proposição 2.1.1 é direcionada para modelos de filas, mas podemos utilizar a idéia no contexto do nosso modelo. Começamos observando que o espaço de parâmetros de $\left\{N_{t}(x, y):-\infty<x<y<\infty\right\}$ é $\mathbb{R}$, ao contrário da Proposição 2.1.1, que é $[0, \infty)$. Para aplicar a Proposição 2.1.1 vamos classificar os eventos como sendo do tipo i, $i \in \mathbb{Z}$, se uma partícula alcançou o intervalo $\left(a_{i}, b_{i}\right]$, após decorrido um tempo $t$. Adicionalmente, suporemos que $\left\{\left(a_{i}, b_{i}\right]: i \in \mathbb{Z}\right\}$ é seqüência de intervalos disjuntos. Assim, pela 
Proposição 2.1.1, o número de partículas nestes intervalos são v.a's independentes, e portanto, o processo $\left\{N_{t}(x, y):-\infty<x<y<\infty\right\}$ tem incrementos independentes.

A probabilidade de uma partícula que partiu de $x \in \mathbb{R}$ alcançar o intervalo $\left(a_{i}, b_{i}\right]$, após um tempo $t$, é

$$
P_{t}^{x}(i)=\int_{a_{i}-x}^{b_{i}-x} \frac{1}{\sqrt{2 \pi t}} \exp \left\{-\frac{y^{2}}{2 t}\right\} d y .
$$

Por (2.1.1), o número médio de partículas em $\left(a_{i}, b_{i}\right]$ é

$$
\begin{aligned}
1 \times \int_{-\infty}^{\infty} P_{t}^{x}(i) d x & =\int_{-\infty}^{\infty} \int_{a_{i}-x}^{b_{i}-x} \frac{1}{\sqrt{2 \pi t}} \exp \left\{-\frac{y^{2}}{2 t}\right\} d y d x \\
& =\int_{-\infty}^{\infty} \int_{a_{i}}^{b_{i}} \frac{1}{\sqrt{2 \pi t}} \exp \left\{-\frac{(z-x)^{2}}{2 t}\right\} d z d x \\
& =\int_{a_{i}}^{b_{i}}\left(\int_{-\infty}^{\infty} \frac{1}{\sqrt{2 \pi t}} \exp \left\{-\frac{(x-z)^{2}}{2 t}\right\} d x\right) d z \\
& =\int_{a_{i}}^{b_{i}} d z=b_{i}-a_{i}
\end{aligned}
$$

sendo que a integral em $x$ é igual a 1 pelo fato de se tratar de uma densidade de uma distribuição normal de média $z$ e variância $t$. Portanto, o número médio de partículas é igual à amplitude do intervalo $\left(a_{i}, b_{i}\right]$. Concluimos que $\left\{N_{t}(x, y):-\infty<x<y<\infty\right\}$ é Processo de Poisson homogêneo de taxa 1.

\subsection{Passeio Aleatório Simples como uma aproxima- ção discreta ao Movimento Browniano}

Vamos assumir que partículas realizam um Passeio Aleatório unidimensional com deslocamento $-\Delta$ à esquerda e $\Delta$ à direita, $\Delta \in(0,1)$, com probabilidades $\frac{1}{2}$. Assim, cada partícula se desloca de acordo com um Passeio Aleatório Simples independente de incremento $\Delta$, em intervalos de tempo de comprimento $\Delta^{2}$. Suponha que no instante 
inicial uma partícula esteja na posição $x=0$. Após um tempo $t>0$ ela terá realizado aproximadamente $n=\left\lfloor\frac{t}{\Delta^{2}}\right\rfloor$ deslocamentos independentes, representados pelas variáveis $\xi_{1}^{\Delta}, \xi_{2}^{\Delta}, \ldots, \xi_{n}^{\Delta}$, tais que $P\left(\xi_{i}^{\Delta}=-\Delta\right)=P\left(\xi_{i}^{\Delta}=\Delta\right)=\frac{1}{2}$. A posição da partícula após $n$ deslocamentos é dada por:

$$
X_{n}^{\Delta}:=\sum_{i=1}^{n} \xi_{i}^{\Delta}
$$

Pelo Teorema Central do Limite, para $\Delta$ suficientemente pequeno, a lei de $X_{n}^{\Delta}$ será aproximadamente normal de média zero e variância $t$.

Agora vamos definir o processo:

$$
Y_{t}^{\Delta}:=X_{\left\lfloor\Delta^{-2} t\right\rfloor}^{\Delta}+\left(\Delta^{-2} t-\left\lfloor\Delta^{-2} t\right\rfloor\right) \xi_{\left\lfloor\Delta^{-2} t\right\rfloor+1}^{\Delta}, \quad t \geq 0 .
$$

O processo em (2.2.1) é a tempo discreto, enquanto (2.2.2) é a tempo contínuo, obtido por interpolação linear, ou seja, para $k$ inteiro positivo tal que $k<\Delta^{-2} t<k+1, Y_{t}^{\Delta}$ é a interpolação entre $X_{k}^{\Delta}$ e $X_{k+1}^{\Delta}$. Além disso,

$$
\begin{aligned}
E\left(Y_{t}^{\Delta}-Y_{s}^{\Delta}\right) & =0 \\
\operatorname{Var}\left(Y_{t}^{\Delta}-Y_{s}^{\Delta}\right) & =t-s .
\end{aligned}
$$

Em (2.2.3) e (2.2.4), tome $m=\Delta^{-2} t$ e $k=\Delta^{-2} s$, com $m>k \geq 0$ inteiros. Então,

$$
Y_{t}^{\Delta}-Y_{s}^{\Delta}=\xi_{k+1}^{\Delta}+\cdots+\xi_{m}^{\Delta}
$$

De (2.2.5) temos

$$
\begin{aligned}
E\left(Y_{t}^{\Delta}-Y_{s}^{\Delta}\right) & =0 ; \\
\operatorname{Var}\left(Y_{t}^{\Delta}-Y_{s}^{\Delta}\right) & =\sum_{j=k+1}^{m} \operatorname{Var}\left(\xi_{j}^{\Delta}\right)=\Delta^{2}\left(\frac{t}{\Delta^{2}}-\frac{s}{\Delta^{2}}\right)=t-s .
\end{aligned}
$$


Das considerações anteriores concluimos que $\left\{Y_{t}^{\Delta}\right\}_{t \geq 0}$ tem incrementos independentes, esperança zero e variância igual ao comprimento do intervalo, isto é, mesmas características do Movimento Browniano. Pelo Princípio da Invariância de Donsker (ver [12]), para $0 \leq t_{1}, \ldots, t_{d}<\infty$ (d inteiro positivo), quando $\Delta \downarrow 0$ o vetor $\left(Y_{t_{1}}^{\Delta}, \ldots, Y_{t_{d}}^{\Delta}\right)$ converge fracamente para $\left(B_{t_{1}}, \ldots, B_{t_{d}}\right)$, sendo $\left\{B_{t}: t \geq 0\right\}$ Movimento Browniano padrão unidimensional. Também, pelo Teorema 1.7.1, temos uma aproximação no sentido forte (quase certamente).

\subsection{Simulando o Movimento Browniano}

Como vimos na seção 2.2, as trajetórias das somas parciais no Passeio Aleatório Simples imitam muito bem o Movimento Browniano se os incrementos de tempo e espaço são suficientemente pequenos. Isto nos fornece um método para simular o Movimento Browniano.

No processo do Capítulo 1 a distância entre dois sítios vizinhos era igual a 1 e a taxa de salto de cada partícula também igual a 1. Além disso, uma partícula só podia saltar à direita. Agora façamos com que a distância entre dois sítios vizinhos mais próximos seja $\Delta \in(0,1)$ e que cada partícula possa se deslocar à esquerda ou direita com probabilidade $\frac{1}{2}$, respectivamente. Também vamos admitir que no instante inicial, em cada sítio diferente do zero, seja atribuída de maneira independente uma partícula com probabilidade $\triangle$. Quanto ao sítio zero, este obrigatoriamente começará com uma partícula.

Os incrementos de tempo serão iguais a $\Delta^{2}$. Desta maneira, decorrido um tempo $t$, terá ocorrido aproximadamente $n=\left\lfloor\frac{t}{\Delta^{2}}\right\rfloor$ deslocamentos. Logo, para cada $\Delta$ fixado teremos $X_{n}^{\Delta}$, definido em (2.2.1), representando a posição da partícula. Como vimos na seção 2.2 , tomando $\Delta \downarrow 0$ teremos a aproximação ao Movimento Browniano.

Para $\Delta \in(0,1)$ fixado, o conjunto de sítios será $\{\ldots,-2 \Delta,-\Delta, 0, \Delta, 2 \Delta, \ldots\}$. 
Fazendo $\Delta \downarrow 0$ teremos este conjunto convergindo para $\mathbb{R}$. Além disso, uma vez que no instante inicial em cada sítio é atribuída uma partícula com probabilidade $\Delta$, tomando $\Delta$ suficientemente pequeno a configuração inicial convergirá para um Processo de Poisson Homogêneo de taxa 1, condicionado a uma partícula no sítio zero. Verifiquemos isto. Denotemos por $\left\{N_{0}(x, y):-\infty<x<y<\infty\right\}$ o processo de contagem do número de partículas no intervalo $(x, y]$, no instante inicial. Como partículas são atribuídas de maneira independente nos sítios segue que os incrementos são independentes. Já, a probabilidade de $k$ partículas no intervalo $(x, x+h]$ depende apenas de $h$, logo, os incrementos são estacionários.

Seja o intervalo $(x, x+h]$. Para $\Delta \in(0,1)$ teremos aproximadamente $\frac{h}{\Delta}$ sítios neste intervalo. Denotando $n=\left\lfloor\frac{h}{\Delta}\right\rfloor$, para $k=0, \ldots, n$,

$$
\begin{aligned}
P\left(N_{0}(x, x+h)=k\right) \approx & \left(\begin{array}{l}
n \\
k
\end{array}\right)\left(\frac{h}{n}\right)^{k}\left(1-\frac{h}{n}\right)^{n-k} \\
= & \frac{n(n-1) \cdots(n-k+1)}{n^{k}} \times \\
& \times\left(\frac{h^{k}}{k !}\right)\left(1-\frac{h}{n}\right)^{n}\left(1-\frac{h}{n}\right)^{-k} .
\end{aligned}
$$

Em (2.3.1), $\triangle \downarrow 0$ implica em $n \rightarrow \infty$, portanto,

$$
\lim _{\Delta \downarrow 0} P\left(N_{0}(x, x+h)=k\right)=e^{-h} \frac{h^{k}}{k !} .
$$

Logo, $\left\{N_{0}(x, y):-\infty<x<y<\infty\right\}$ convergirá para um Processo de Poisson Homogêneo de taxa 1 .

Para fins de simulação será preciso considerar uma caixa (intervalo) finita e um tempo pré-determinado de simulação. Para tal consideramos o segmento de reta $\left[-\frac{M}{4}, \frac{5}{4} M\right], M>0$, e um tempo máximo $T=\frac{M}{\Delta^{2}}$. Neste intervalo serão alocados $\frac{1}{\Delta}$ pontos cuja distância entre eles será $\Delta$. Sendo assim, segundo a configuração inicial que estabelecemos anteriormente, o número esperado de partículas será $1+\left(\frac{\frac{3}{2} M}{\Delta}\right) \times \Delta=$ 
$1+\frac{3}{2} M$

Note que assumimos como extremos $-\frac{M}{4}$ e $\frac{5}{4} M$. Dessa maneira haverá menos sítios negativos e, conseqüentemente, um menor número de partículas contaminadas. Com isto estaremos dificultando a propagação da contaminação, dando uma maior vantagem para as normais no instante inicial.

A estimativa para a velocidade de contaminação será $\frac{Z_{T}}{L}, L$ o extremo superior da caixa. A interpretação deste quociente é a taxa com que $Z_{t}, 0 \leq t \leq T$, vai alcançar $L$.

\subsection{O algoritmo para a simulação}

O detalhamento do algoritmo segue abaixo:

(1o) Atribua um valor para $M>0$ e $\Delta \in(0,1)$.

(2o) $t \longleftarrow 0$, que é o instante inicial, $T \longleftarrow \frac{M}{\Delta^{2}}$.

(3o) $n \longleftarrow\left\lceil\frac{M}{4 \Delta}\right\rceil, m \longleftarrow\left\lceil\frac{5 M}{4 \Delta}\right\rceil$.

(4o) $l \longleftarrow-n \Delta$ é a cota inferior da caixa e $L \longleftarrow m \Delta$ a cota superior.

(5o) Crie o vetor Eta de dimensão $d=m+n+1$, que armazenará a quantidade de partículas nos sítios. As posições (índices) de 1 até $n$ são referentes aos sítios negativos, a posição $n+1$ é reservada para o sítio zero e a de $n+2$ até $d$ para os sítios positivos. Obrigatoriamente o sítio zero deverá começar com 1 partícula. Nos sítios restantes atribua independentemente partículas segundo uma distribuição de Bernoulli de parâmetro $\Delta$.

(6o) Crie o vetor $V$ que conterá a posição dos sítios ocupados.

(7o) No instante inicial todos os sítios menores ou iguais a zero serão declarados 
contaminados e os maiores que zero normais. Assim, $z=0$ será a fronteira inicial entre contaminados e normais.

(80) Todas partículas irão realizar um salto, com probabilidade $\frac{1}{2}$ à direita e $\frac{1}{2}$ à esquerda. Havendo mais do que uma partícula num sítio, então todas irão saltar. Para o sítio referente à cota inferior o salto permitido é apenas à direita e o da cota superior somente à esquerda. Se uma partícula do sítio $z$ saltar à direita, então todas lá ficarão contaminadas e $z \longleftarrow z+1$.

(9o) Atualize o vetor V.

(10o) Se $t \leq T$ faça $t \longleftarrow t+1$ e volte para (8o), senão apresente os resultados $z$ e $\frac{z}{L}$, que são, respectivamente, a fronteira e a velocidade de contaminação após o término da simulação.

\subsection{Programa em Scilab}

Para executar o programa é necessário atribuir o parâmetro para o tamanho da caixa ( variável $M$ ), dos subintervalos (no programa a variável delta corresponde a $\Delta$ mencionada na seção 2.4) e o número de simulações (N).

\section{Listagem do programa em Scilab:}

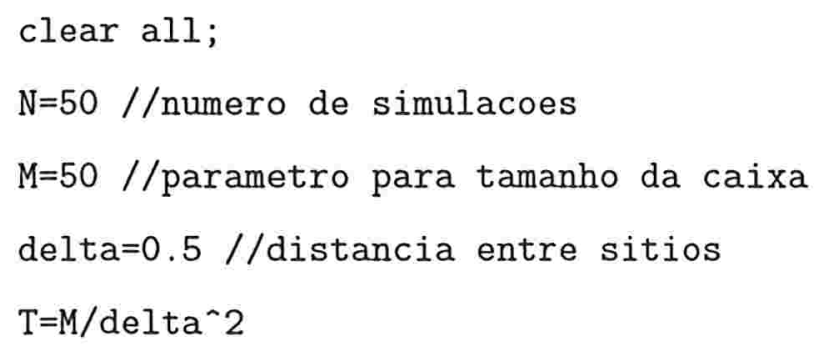




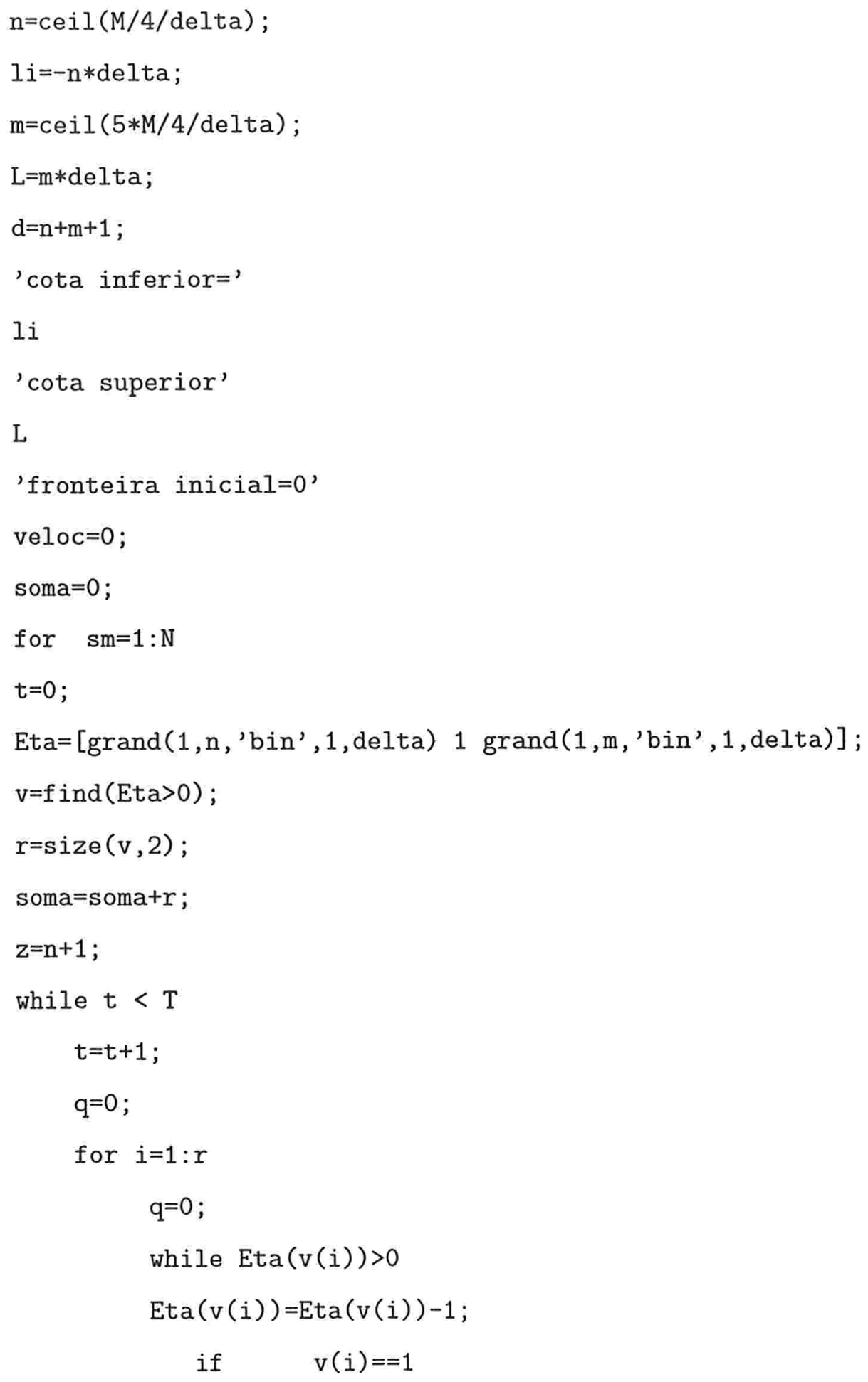




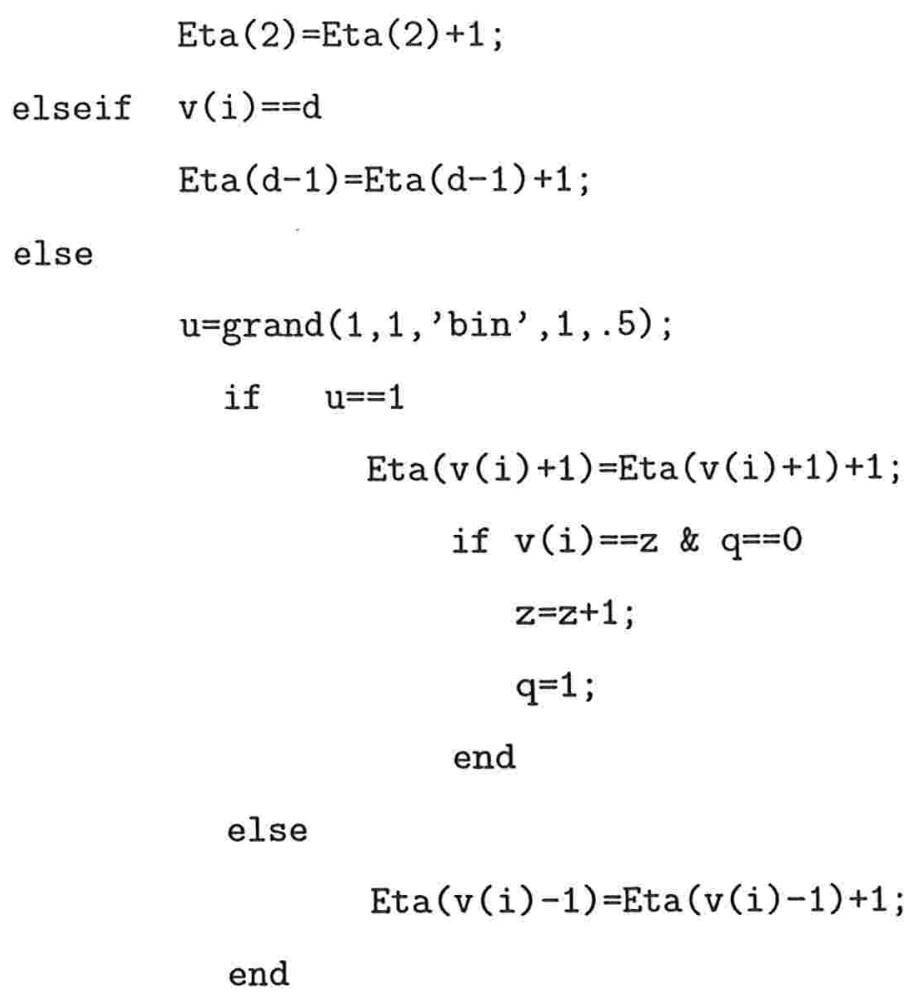




\subsection{Alguns exemplos da execução do programa}

A seguir apresentamos os resultados de algumas simulações. Como podemos observar, a média das velocidades fica aproximadamente em $\frac{1}{2}$, reforçando a conjectura que fizemos em (1.7.8).

$M=30$

$\mathrm{N}=20$

delta $=0.05$

$\mathrm{T}=12000$

cota inferior da caixa $=-7.5$

cota superior da caixa $=37.5$

média das velocidades $=0.5200$

numero médio de partículas $=46.4$

$M=50$

$\mathrm{N}=10$

$\operatorname{delta}=0.1$

$\mathrm{T}=5000$

cota inferior da caixa $=-12.5$

cota superior da caixa $=62.5$

média das velocidades $=0.4962$

numero médio de partículas $=73.63$ 
$M=70$

$\mathrm{N}=10$

delta $=0.1$

$\mathrm{T}=7000$

cota inferior da caixa $=-17.5$

cota superior da caixa $=87.5$

média das velocidades $=0.5145$

numero médio de partículas $=108.3$

$M=100$

$\mathrm{N}=10$

$\operatorname{delta}=0.1$

$\mathrm{T}=10000$

cota inferior da caixa $=-25$

cota superior da caixa $=125$

média das velocidades $=0.5127$

numero médio de partículas $=156$

$M=50$

$\mathrm{N}=50$

delta $=0.5$

$\mathrm{T}=200$

cota inferior da caixa $=-12.5$ 
cota superior da caixa $=62.5$

média das velocidades $=0.5206$

numero médio de partículas $=72.5$

$M=70$

$\mathrm{N}=40$

delta $=0.5$

$\mathrm{T}=280$

cota inferior da caixa $=-17.5$

cota superior da caixa $=87.5$

média das velocidades $=0.5067$

numero médio de partículas $=107.375$

$M=100$

$\mathrm{N}=40$

delta $=0.5$

$\mathrm{T}=400$

cota inferior da caixa $=-25$

cota superior da caixa $=125$

média das velocidades $=0.4886$

numero médio de partículas $=153.35$ 


\subsection{Programa com opção de gráfico}

Uma outra versão para o programa anterior inclui a opção de gráfico para fronteira $\times$ tempo. Este programa executa apenas uma simulação. Os parâmetros a serem fornecidos são M, delta e op. Se op=1 então serão apresentados os sítios ocupados nos instantes inicial e final.

Listagem do programa em Scilab, com opção de gráfico:

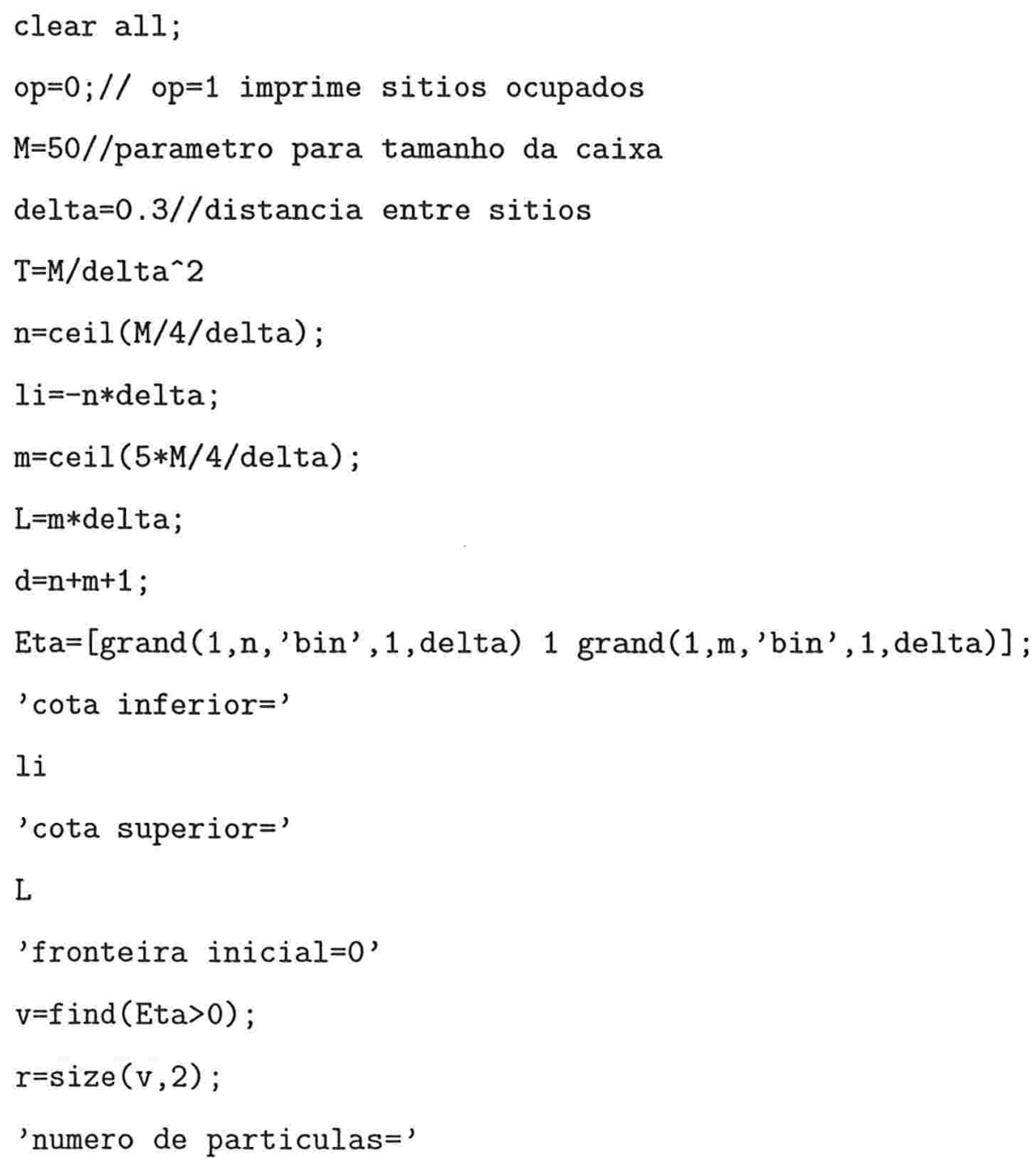




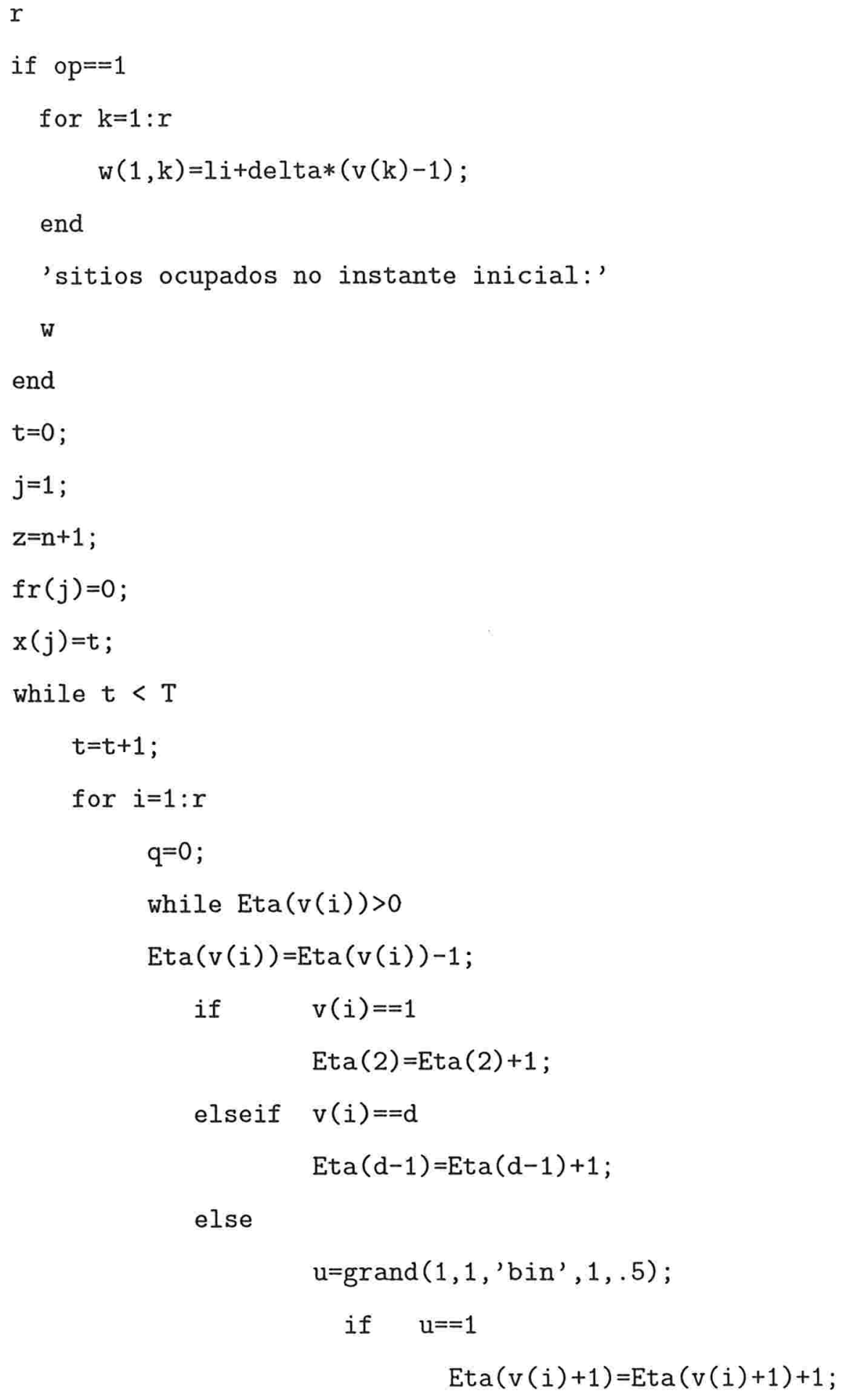




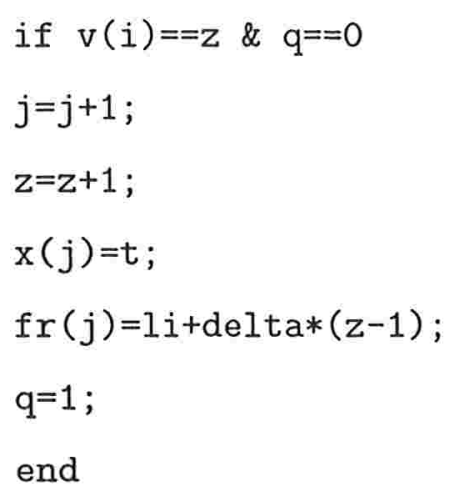


$\operatorname{fr}(j) / L$

plot (x,fr, 'instante', 'fronteira')

Como exemplo executamos o programa com os valores $M=50$, delta $=0.3$ e op $=0$. Os resultados foram:

$\mathrm{T}=555.56$

cota inferior $=-12.6$

cota superior $=62.7$

fronteira inicial $=0$

número de partículas $=75$

fronteira no instante final $=31.5$

velocidade $=0.5024$

A Figura 2.2 mostra o gráfico correspondente.

\subsection{Conclusões}

Verificamos empiricamente que a velocidade de contaminação em Movimentos Brownianos independentes é próxima de $\frac{1}{2}$, estando de acordo com os resultados obtidos no capítulo 1. Outro resultado foi que a medida invariante do número de partículas em $\mathbb{R}$ segue um Processo de Poisson Homogêneo de taxa 1 , dado que no instante inicial também se começou com este mesmo processo. 


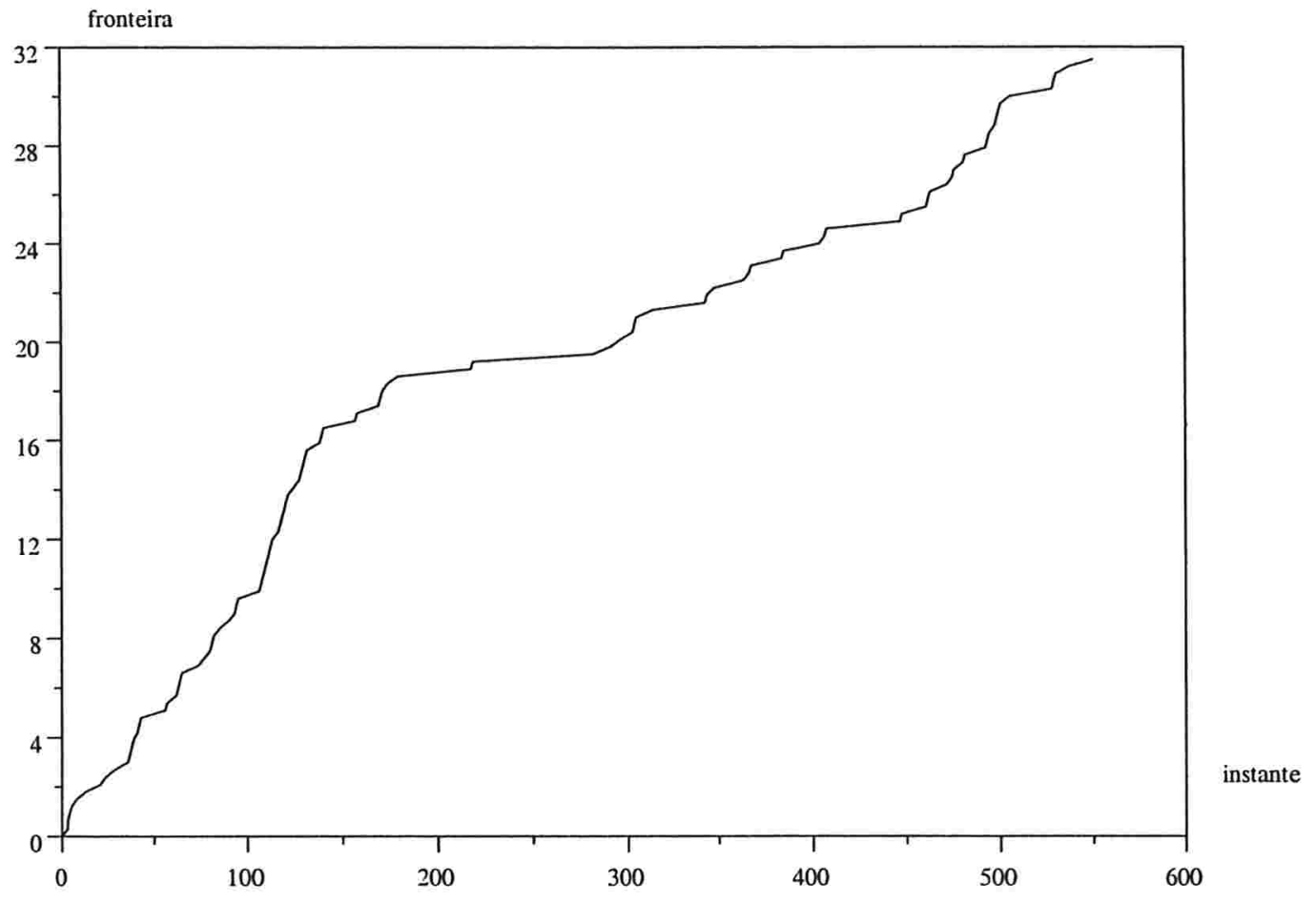

Figura 2.2: Evolução da fronteira 


\section{Capítulo 3}

\section{Modelo com partículas ativas nos sítios contaminados e inativas nos}

\section{normais}

\subsection{Definindo o modelo}

Começamos definindo o que denominaremos de modelo geral. Aqui os sítios são o conjunto dos inteiros. No instante inicial o sítio zero obrigatoriamente deverá conter pelo menos uma partícula contaminda. Sendo assim, a configuração inicial no zero será $1+\eta(0)$, com $\eta(0) \sim \operatorname{Pois}(\lambda)$. Nos outros sítios a configuração inicial será escolhida de acordo com a medida-produto de $\operatorname{Pois}(\lambda)$. Ainda em relação ao instante inicial, partículas de sítios não positivos serão declaradas contaminadas e dos positivos normais.

A dinâmica do processo é a seguinte:

- para cada partícula de um sítio contaminado associamos um Processo de Poisson Homogêneo de taxa 1. Sendo assim, essas partículas se deslocarão da esquerda à direita e os intervalos entre saltos formarão uma seqüência de v.a's iid Expon(1); 
- em um sítio contaminado apenas uma partícula saltará;

- partículas em um sítio normal têm taxa zero de deslocamento;

- no momento em que uma partícula contaminada atingir uma normal, as partículas lá contidas passarão a ter a mesma taxa das contaminadas;

- partículas dentro de um mesmo sítio não interagem entre si, assim como partículas de sítios distintos. Logo, os Processos de Poisson serão mutuamente independentes.

O modelo que estamos propondo é semelhante ao "Frog Model", no qual inicialmente apenas o sítio da origem contém uma partícula contaminada (ativa). A partir do instante inicial esta partícula ativa começa um Passeio Aleatório Simples simétrico e vai contaminando o sistema. Detalhes deste modelo estão em [1], [2] e [19].

Outro processo que definiremos neste capítulo é o modelo truncado. Neste modelo os sítios são $\{0,1, \ldots\}$. Analogamente ao modelo geral, a configuração inicial no sítio zero será $1+\eta(0), \eta(0) \sim$ Pois $(\lambda)$. Nos sítios positivos a configuração inicial seguirá a medida-produto de Pois $(\lambda)$. A dinâmica deste novo modelo será a mesma do modelo geral. A finalidade de introduzir este modelo é que através dele iremos obter conclusões para o modelo geral.

Analogamente aos Capítulos 1 e 2 vamos definir $Z_{t}$ como sendo a fronteira no instante $t$, ou seja, o sítio que no instante atual $t$ separa contaminados dos normais, e também definiremos $v:=\lim _{t \rightarrow \infty} \frac{Z_{t}}{t}$ como a velocidade de propagação da contaminação.

Os resultados principais neste capítulo são a existência da velocidade $v$ e o fato de que esta é maior que 1. Através do Teorema Ergódico Subaditivo, enunciado na próxima seção, obteremos um resultado de existência de $v$ para o modelo truncado. Depois mostraremos que a velocidade no modelo geral é a mesma do truncado. 


\subsection{O Teorema Ergódico Subaditivo}

Através do teorema a seguir iremos obter uma cota inferior para a velocidade $v$. Este teorema é um melhoramento daquele obtido por Kingman (ver [15]).

Teorema 3.2.1. (Teorema Ergódico Subaditivo). Assuma que $\{X(m, n)\}$ é seqüência de v.a's positivas, com $m, m \in \mathbb{N} e 0 \leq m<n$, tal que:

(i) $X(0, n) \leq X(0, m)+X(m, n)$ para todo $0 \leq m<n$;

(ii) a distribuição conjunta de $\{X(m+1, m+k+1): k \geq 1\}$ é a mesma de $\{X(m, m+k): k \geq 1\}$ para $\forall m \geq 0 ;$

(iii) para cada $k \geq 1$ a seqüência $\{X(n k,(n+1) k): n \geq 1\}$ é um processo ergódico estacionário;

(iv) $E(X(0,1))<\infty$.

Portanto,

sendo $\gamma:=\inf _{n \geq 0} \frac{E(X(0, n))}{n}$.

$$
\lim _{n \rightarrow \infty} \frac{X(0, n)}{n}=\gamma \text { quase certamente, }
$$

Prova: [17].

\subsection{Aplicando o Teorema Ergódico Subaditivo}

Aqui apresentamos uma construção formal do modelo truncado, com a finalidade de aplicar o Teorema Ergódico Subaditivo. Seja $T(x, y)$ o tempo necessário para uma partícula contaminada do sítio $x$ alcançar $y$, com $0 \leq x<y<\infty$, ou seja, o tempo para que a infecção alcance $y$, partindo de uma partícula de $x$. Queremos provar que a seqüência $\{T(x, y): 0 \leq x<y<\infty\}$ satisfaz as condições do Teorema 3.2.1, e assim aplicá-lo ao modelo truncado. Para todo sítio $x$ defina $\left\{\mathcal{E}_{1}^{x}, \mathcal{E}_{2}^{x}, \ldots\right\}$ como sendo uma seqüência de v.a's iid com distribuição comum Expon(1). Através desta 


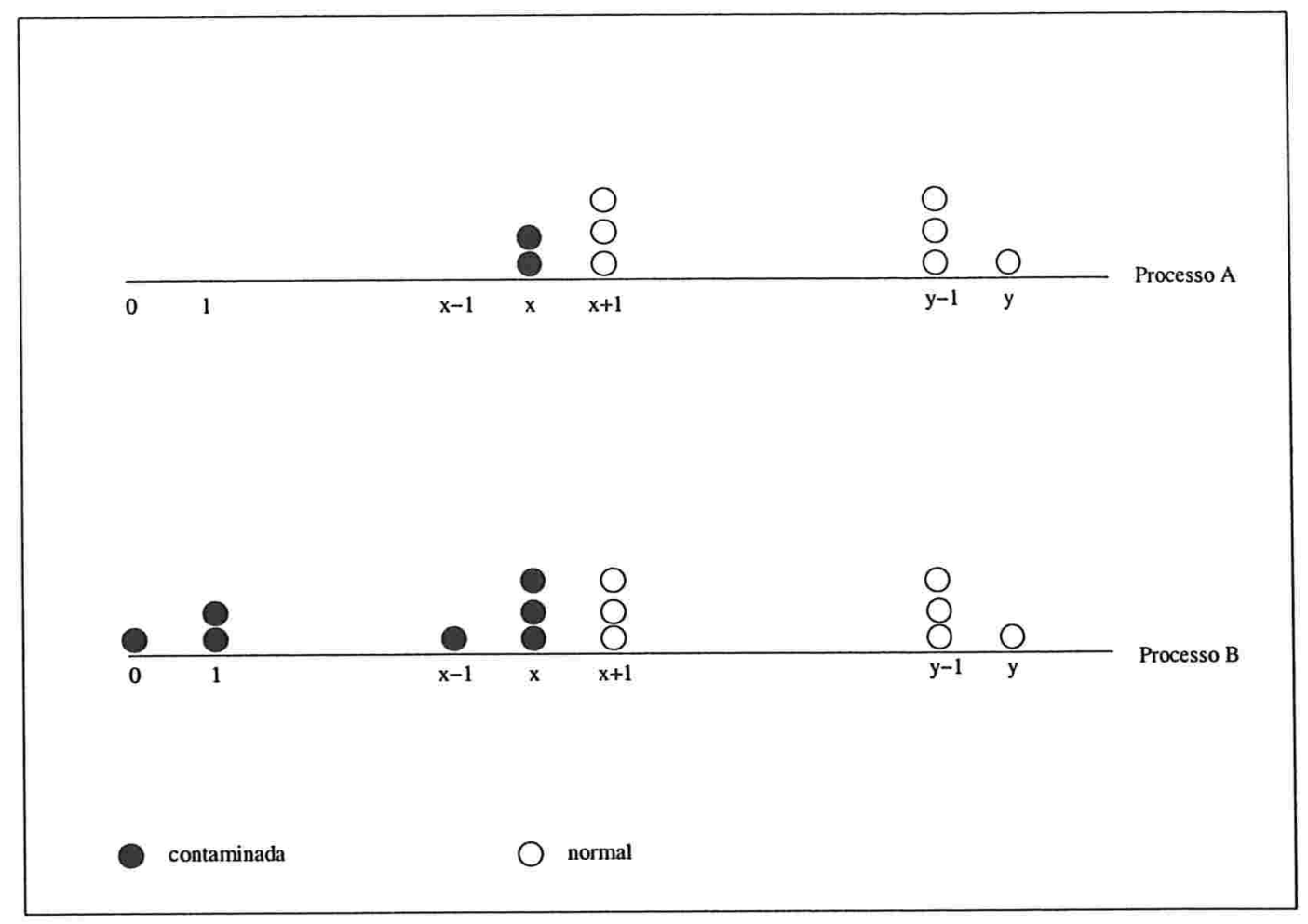

Figura 3.1: Exemplificação dos processos $A$ e $B$

sequiência é que se determinará o tempo de permanência da $i$-ésima partícula em $x$ (lembre que a cada partícula está associado um Processo de Poisson de taxa 1). Com base nesta seqüência construiremos um acoplamento, que consiste em considerar dois processos com a mesma configuração com relação ao número de partículas nos sítios $x+1$ até $y$. Vamos denominar como "Processo $A$ " aquele com nenhuma partícula em $\{0, \ldots, x-1\}$ e pelo menos uma em $x$. O outro processo, denominado "Processo $B$ ", poderá conter partículas em $\{0, \ldots, x-1\}$ e em $x$ deverá conter uma quantidade maior ou igual a do Processo $A$. Além dessas condições, no Processo $A$ o sítio $x$ é contaminado e no $B\{0,1, \ldots, x-1, x\}$ são contaminados. O que os processos $A$ e $B$ têm em comum é a mesma configuração em $\{x+1, x+2, \ldots\}$. A Figura 3.1 exemplifica os processos $A$ e $B$.

No instante inicial, para $x \leq u<y$, definimos: 


$$
\begin{aligned}
& N_{u}^{A}:=\text { "número de partículas no sítio } u \text { no processo } A \text { ", } \\
& N_{u}^{B}:=\text { "número de partículas no sítio } u \text { no processo } B \text { ". }
\end{aligned}
$$

Note que $N_{u}^{B} \geq N_{u}^{A}$, para qualquer $x \leq u<y$. Também defina

$$
\begin{aligned}
& \mathcal{T}_{n}^{A}(u):=\text { "instante em que a } n \text {-ésima partícula saiu do sítio } u \text { no processo } A " \\
& \mathcal{T}_{n}^{B}(u):=\text { "instante em que a } n \text {-ésima partícula saiu do sítio } u \text { no processo } B \text { ". }
\end{aligned}
$$

Lema 3.3.1. Sejam $x \leq u<y$ e $n \leq N_{u}^{A}$. Então, $\mathcal{T}_{n}^{A}(u) \geq \mathcal{T}_{n}^{B}(u)$.

Prova: faremos por indução em $n$. Suponha $u=x$. Então,

$$
\mathcal{T}_{1}^{A}(x)=\min \left\{\mathcal{E}_{j}^{x}: j=1, \ldots, N_{x}^{A}\right\} \text { e } \mathcal{T}_{1}^{B}(x)=\min \left\{\mathcal{E}_{j}^{x}: j=1, \ldots, N_{x}^{B}\right\}
$$

Teremos que $\mathcal{T}_{1}^{B}(x) \leq \mathcal{T}_{1}^{A}(x)$. Agora vamos nos concentrar no processo $A$. Para a segunda partícula em $x, \mathcal{T}_{2}^{A}(x)=\min \left\{\left\{\mathcal{E}_{j}^{x}: j=1, \ldots, N_{x}^{A}\right\}-\left\{\mathcal{T}_{1}^{A}(x)\right\}\right\}$, e assim procedemos até obter $\mathcal{T}_{N_{x}^{A}}^{A}(x)$. Após as partículas do sítio $x$ saltarem, em $x+1$ haverá $m+N_{x}^{A}$, sendo $m$ a quantidade inicial que havia em $x+1$. Aqui introduzimos a notação

$$
\begin{aligned}
\mathcal{C}^{A}(x+1)= & \left\{\mathcal{T}_{1}^{A}(x)+\mathcal{E}_{1}^{x+1}, \mathcal{T}_{1}^{A}(x)+\mathcal{E}_{2}^{x+1}, \ldots, \mathcal{T}_{1}^{A}(x)+\mathcal{E}_{m}^{x+1}, \mathcal{T}_{1}^{A}(x)+\mathcal{E}_{m+1}^{x+1},\right. \\
& \left.\mathcal{T}_{2}^{A}(x)+\mathcal{E}_{m+2}^{x+1}, \mathcal{T}_{3}^{A}(x)+\mathcal{E}_{m+3}^{x+1}, \ldots, \mathcal{T}_{N_{x}^{A}}^{A}(x)+\mathcal{E}_{m+N_{x}^{A}}^{x+1}\right\}
\end{aligned}
$$

No processo $A$, o instante da primeira partícula que saltará de $x+1$ será $\mathcal{T}_{1}^{A}(x+1)=$ $\min \left\{\mathcal{C}^{A}(x+1)\right\}$. Para a segunda partícula, em $\mathcal{C}^{A}(x+1)$ excluimos $\mathcal{T}_{1}^{A}(x+1)$ e tomamos o próximo menor valor, ou seja, $\mathcal{T}_{2}^{A}(x+1)=\min \left\{\mathcal{C}^{A}(x+1)-\mathcal{T}_{1}^{A}(x+1)\right\}$. Desta maneira 
procedemos até obter $\mathcal{T}_{m+N_{x}^{A}}^{A}(x+1)$. Supondo que $N_{x}^{B}=N_{x}^{A}$, de maneira análoga, no Processo $B$ teremos o conjunto

$$
\begin{aligned}
\mathcal{C}^{B}(x+1)= & \left\{\mathcal{T}_{1}^{B}(x)+\mathcal{E}_{1}^{x+1}, \mathcal{T}_{1}^{B}(x)+\mathcal{E}_{2}^{x+1}, \ldots, \mathcal{T}_{1}^{B}(x)+\mathcal{E}_{m}^{x+1}, \mathcal{T}_{1}^{B}(x)+\mathcal{E}_{m+1}^{x+1},\right. \\
& \left.\mathcal{T}_{2}^{B}(x)+\mathcal{E}_{m+2}^{x+1}, \mathcal{T}_{3}^{B}(x)+\mathcal{E}_{m+3}^{x+1}, \ldots, \mathcal{T}_{N_{x}^{A}}^{B}(x)+\mathcal{E}_{m+N_{x}^{A}}^{x+1}\right\}
\end{aligned}
$$

Procedendo como antes obteremos os instantes

$$
\mathcal{T}_{1}^{B}(x+1), \mathcal{T}_{2}^{B}(x+1), \ldots, \mathcal{T}_{m+N_{x}^{A}}^{B}(x+1)
$$

Se caso $N_{x}^{B}>N_{x}^{A}$, ainda teremos

$$
\mathcal{T}_{m+N_{x}^{A}+1}^{B}(x+1), \ldots \ldots, \mathcal{T}_{m+N_{x}^{B}}^{B}(x+1) .
$$

Usando o fato que $\mathcal{T}_{1}^{B}(x) \leq \mathcal{T}_{1}^{A}(x)$ e a hipótese de indução $\mathcal{T}_{n}^{B}(x) \leq \mathcal{T}_{n}^{A}(x)$ teremos

$$
\begin{aligned}
& \mathcal{T}_{1}^{A}(x)+\mathcal{E}_{1}^{x+1} \geq \mathcal{T}_{1}^{B}(x)+\mathcal{E}_{1}^{x+1}, \mathcal{T}_{1}^{A}(x)+\mathcal{E}_{2}^{x+1} \geq \mathcal{T}_{1}^{B}(x)+\mathcal{E}_{2}^{x+1}, \ldots, \\
& \mathcal{T}_{N_{x}^{A}}^{A}(x)+\mathcal{E}_{m+N_{x}^{A}}^{x+1} \geq \mathcal{T}_{N_{x}^{A}}^{B}(x)+\mathcal{E}_{m+N_{x}^{A}}^{x+1}
\end{aligned}
$$

e conseqüentemente $\mathcal{T}_{n}^{A}(x+1) \geq \mathcal{T}_{n}^{B}(x+1)$, para $n \in\left\{1, \ldots, m+N_{x}^{A}\right\}$. De maneira análoga, mostra-se que $\mathcal{T}_{n}^{A}(u) \geq \mathcal{T}_{n}^{B}(u)$ para $x<u<y$ e $n \leq N_{u}^{A}$.

Lema 3.3.2. A seqüência $\{T(x, y): x, y \in \mathbb{Z} e 0 \leq x<y\}$ satisfaz as hipóteses do Teorema 3.2.1.

\section{Prova:}

(i) No Lema 3.3.1 tome $n=1$ e $u=y-1$. Então, $T(x, y)=\mathcal{T}_{1}^{A}(y-1)$. Mas, para comparar com o tempo que o processo $A$ leva para alcançar $y$, no processo $B$ precisamos descontar o tempo necessário até alcançar $x$, ou seja, $T(0, y)-T(0, x)=\mathcal{T}_{1}^{B}(y-1)$. Assim, $T(x, y) \geq T(0, y)-T(0, x)$, isto é, $T(0, y) \leq T(0, x)+T(x, y)$. 
(ii) Vamos definir $E_{\eta}$ e $P_{\eta}$ como a esperança e a probabilidade, respectivamente, para $T(0, x)$ com a configuração inicial $\eta$ fixada. Também definimos $\mathbf{E}$ e $\mathbf{P}$ como a esperança e a probabilidade com relação à configuração inicial $\eta$. Além disso, $\mathbb{E}=\mathbf{E} E_{\eta}$ e $\mathbb{P}=\mathbf{P} P_{\eta}$ são a esperança e a probabilidade totais para $T$, ou seja, podem ser interpretadas como uma probabilidade total, sendo $P_{\eta}$ vista como uma probabilidade condicionada à configuração inicial $\eta$.

Agora considere que no sítio zero haja $\eta(0)+1$ partículas e no sítio $1 \eta(1)$, com $\eta(0)+1 \neq \eta(1)$. Segue que a lei de $T(0,1)$ será $\operatorname{Expon}(\eta(0)+1)$ e a de $T(1,2) \sim$ $\operatorname{Expon}(\eta(1))$. Assim, $T(0,1)$ e $T(1,2)$ não terão mesma lei para uma configuração inicial $\eta$ fixada, e isto implicará que $\{T(x, x+k): k \geq 1\}$ e $\{T(x+1, x+k+1): k \geq 1\}$ não terão mesma lei no contexto $\left\{P_{\eta}, E_{\eta}\right\}$. Por outro lado,

$$
\begin{aligned}
\mathbb{P}(T(0,1)>t) & =\sum_{n=1}^{\infty} \mathbf{P}(\eta(0)=n-1) P_{\eta}(T(0,1)>t \mid \eta(0)=n-1) \\
& =\sum_{n=1}^{\infty}\left(e^{-\lambda} \frac{\lambda^{n-1}}{(n-1) !}\right) e^{-(n-1) t} \\
& =\exp \left\{\lambda\left(e^{-t}-1\right)\right\}
\end{aligned}
$$

e analogamente,

$$
\begin{aligned}
\mathbb{P}(T(1,2)>t) & =\sum_{n=0}^{\infty} \mathbf{P}(\eta(1)=n) P_{\eta}(T(1,2)>t \mid \eta(1)=n) \\
& =\exp \left\{\lambda\left(e^{-t}-1\right)\right\}
\end{aligned}
$$

levando-nos a concluir que $T(0,1)$ e $T(1,2)$ terão mesma lei no contexto $\{\mathbb{P}, \mathbb{E}\}$. Pelo mesmo argumento, $\{T(x, x+k): k \geq 1\}$ e $\{T(x+1, x+k+1): k \geq 1\}$ terão mesma lei em $\{\mathbb{P}, \mathbb{E}\}$.

(iii) Para $k \geq 1$, inteiro fixado, 


$$
\begin{aligned}
& T(x k,(x+1) k) \sim \operatorname{Expon}(\eta(x k)), T((x+1) k,(x+2) k) \sim \operatorname{Expon}(\eta((x+1) k)), \\
& \ldots, T((x+m) k,(x+m+1) k) \sim \operatorname{Expon}(\eta((x+m) k)), \ldots
\end{aligned}
$$

Podemos notar que $T((x+m) k,(x+m+1) k)$ tem lei Expon $(\eta((x+m) k))$, ou seja, a lei independe da configuração dos sítios distintos de $(x+m) k$. Assim,

$$
T((x+m) k,(x+m+1) k)
$$

é independente dos outros $T((x+n) k,(x+n+1) k), n \neq m$. Logo,

$$
\{T(x k,(x+1) k): x \geq 1\}
$$

é uma sequiência de v.a's iid, e portanto, estacionária ergódica.

(iv) A configuração incial do sítio zero é $\eta(0)+1$, com $\eta(0) \sim \operatorname{Pois}(\lambda)$. Como cada partícula contaminada tem taxa 1, $T(0,1) \sim \operatorname{Expon}(\eta(0)+1)$. Assim

$$
E_{\eta} T(0,1)=\frac{1}{\eta(0)+1}
$$

e

$$
\begin{aligned}
\mathbb{E} T(0,1) & =\mathbf{E} E_{\eta} T(0,1) \\
& =\mathbf{E}\left(\frac{1}{\eta(0)+1}\right) \\
& =\sum_{j=0}^{\infty} \frac{1}{j+1} e^{-\lambda} \frac{\lambda^{j}}{j !} \\
& =\frac{e^{-\lambda}}{\lambda} \sum_{m=1}^{\infty} \frac{\lambda^{m}}{m !} \\
& =\frac{1-e^{-\lambda}}{\lambda}<1 .
\end{aligned}
$$


Lema 3.3.3. Para a seqüencia $\{T(0, x): x, y \in \mathbb{Z}$ e $0 \leq x<y\}$ temos que

$$
0<\inf _{x \geq 0} \frac{E(T(0, x))}{x}<1
$$

Prova: como no Teorema 3.2.1, faça $\gamma:=\inf _{x \geq 0} \frac{E(T(0, x))}{x}$. Começamos provando que $\gamma>0$. Sabemos que uma partícula contaminada ao atingir um sítio normal irá contaminá-lo. Como conseqüência, o sítio que foi contaminado irá conter $1+$ [número de partículas do sítio]. Este mecanismo é semelhante ao processo de ramificação, o qual em cada geração um indivíduo poderá gerar descendentes ou não. Ainda com relação ao processo de ramificação, se um indivíduo gerar descendentes, então no instante atual teremos uma quantidade de partículas igual a [1 + número de filhos]. Por outro lado, no nosso modelo apenas a primeira partícula contaminada irá tornar as normais iguais a ela. Outras partículas contaminadas ao atingirem este sítio já contaminado não causarão nenhum efeito, ao contrário do processo de ramificação, o qual cada partícula tem probabilidade de gerar descendentes. É nesse sentido que dizemos que nosso modelo é dominado pelo processo de ramificação.

Aqui introduzimos o processo de ramificação com a mesma configuração do modelo truncado. Como no modelo truncado, cada partícula terá associado um Processo de Poisson de taxa 1. Defina

$N_{t}^{r}:=$ "número total de partículas ativas no processo de ramificação no instante $t$ ", e também $H(t):=E N_{t}^{r}$. Como a fronteira inicial é o sítio zero, cuja configuração inicial é $1+\eta_{0}(0), \eta_{0}(0) \sim \operatorname{Pois}(\lambda)$, então $H(0)=E N_{0}^{r}=E\left(1+\eta_{0}(0)\right)=1+\lambda$. Mas, para $h$ suficientemente pequeno,

$$
H(t+h)=E\left(\sum_{i=1}^{N_{t}^{r}} V_{i}\right)
$$

onde 


$$
V_{i}:=\left\{\begin{array}{ll}
1, & \text { se a } i \text {-ésima partícula de } Z_{t} \text { não saltou } \\
1+\lambda, & \text { caso contrário }
\end{array} .\right.
$$

A interpretação de $V_{i}$ é que caso a $i$-ésima partícula de $Z_{t}$ saltar então o sítio $Z_{t}+1$ ficará com $1+\lambda$, pois antes do salto, na média, $Z_{t}+1$ estava com $\lambda$ partículas normais (lembre que a configuração inicial é Pois $(\lambda)$ ). Também podemos pensar que, na média, o processo de ramificação irá gerar $\lambda$ descendentes contaminados, caso uma partícula em $Z_{t}$ saltar.

Note que, para $h$ pequeno, no intervalo $[h, t+h]$ apenas uma partícula saltará. Pelo fato de cada partícula ter um processo de Poisson associado, a probabilidade de salto é proporcional a $1 \times h+\mathrm{o}(h)$. Logo,

$$
E V_{i}=1 \times(1-h)+(1+\lambda) \times h+\overline{\mathrm{o}}(h) \approx 1+\lambda \times h .
$$

Voltando a 3.3.1, como $N_{t}^{r}$ é independente de $\left\{V_{i}\right\}_{i \geq 1}$ e esta última é seqüência de v.a's iid, pela fórmula de Wald,

$$
H(t+h)=E N_{t}^{r} E V_{1}=H(t)(1+h \lambda)=H(t)+\lambda h H(t) .
$$

Disto temos que

$$
\begin{aligned}
H^{\prime}(t) & =\lim _{h \downarrow 0} \frac{H(t+h)-H(t)}{h} \\
& =\lim _{h \downarrow 0} \frac{H(t)+\lambda h H(t)-H(t)}{h} \\
& =\lambda H(t) .
\end{aligned}
$$

Com a condição inicial $H(0)=1+\lambda$, a solução da equação diferencial (3.3.2) é $H(t)=$ $(1+\lambda) e^{\lambda t}$. Pela desigualdade de Chebyshev,

$$
P\left(N_{t}^{r}>2(1+\lambda) e^{\lambda t}\right) \leq \frac{H(t)}{2(1+\lambda) e^{\lambda t}}=\frac{1}{2}
$$


e portanto,

$$
P\left(N_{t}^{r} \leq 2(1+\lambda) e^{\lambda t}\right) \geq \frac{1}{2}
$$

Retornando ao modelo truncado,

$N_{t}:=$ "número total de partículas ativas no modelo truncado no instante $t$ ".

Então,

$$
P\left(N_{t} \leq 2(1+\lambda) e^{\lambda t}\right) \geq P\left(N_{t}^{r} \leq 2(1+\lambda) e^{\lambda t}\right),
$$

e conseqüentemente, por (3.3.3)

$$
P\left(N_{t} \leq 2(1+\lambda) e^{\lambda t}\right) \geq \frac{1}{2}
$$

Agora fixe uma partícula e defina

$$
Y_{t}:=\text { "número de saltos que uma partícula realizou até o instante } t \text { ". }
$$

Observe que $Y_{t} \sim \operatorname{Pois}(t)$. Para $a>0$ e $s>0$, utilizando a desigualdade de Chebyshev,

$$
\begin{aligned}
P\left(Y_{t}>a t\right) & =P\left(e^{s Y_{t}}>e^{s a t}\right) \\
& \leq \frac{M_{Y_{t}}(s)}{e^{s a t}} \\
& =\left(\frac{\exp \left\{e^{s}-1\right\}}{\exp \{s a\}}\right)^{t} \\
& =\exp \left\{-t\left(s a-e^{s}+1\right)\right\} .
\end{aligned}
$$

Da equação $\frac{d}{d s}\left(-t\left(s a-e^{s}+1\right)\right)=0$ obtemos que o mínimo de $-t\left(s a-e^{s}+1\right)$ ocorre para $s=\ln (a)$. Assim, (3.3.5) é tal que

$$
P\left(Y_{t}>a t\right) \leq \exp \{-t(a \ln (a)-a+1)\}
$$


Seja $\left\{U_{i}\right\}_{i \geq 1}$ v.a's iid com distribuição $\operatorname{Pois}(t)$. A interpretação de $U_{i}$ é a posição que a $i$-ésima partícula vai ocupar, após decorrido um tempo $t$. Também definimos $W_{t}:=\max \left\{U_{i}: i=1, \ldots, N_{t}\right\}$. Para $b>0$, pela independência das $U_{i}$, e também usando o fato de que $U_{i}$ é independente de $N_{t}$ para todo $i$,

$$
\begin{aligned}
P\left(W_{t} \leq b t \mid N_{t} \leq 2(1+\lambda) e^{\lambda t}\right) & =P\left(\bigcap_{1 \leq i \leq N_{t}}\left\{U_{i} \leq b t\right\} \mid N_{t} \leq 2(1+\lambda) e^{\lambda t}\right) \\
& \geq\left(P\left(U_{1} \leq b t\right)\right)^{2(1+\lambda) e^{\lambda t}} .
\end{aligned}
$$

Por (3.3.6),

$$
\begin{aligned}
\left(P\left(U_{1} \leq b t\right)\right)^{2(1+\lambda) e^{\lambda t} \geq} & (1-\exp \{-t(b \ln (b)-b+1)\})^{2(1+\lambda) e^{\lambda t}} \\
= & \exp \{2(1+\lambda) \exp \{\lambda t\} \times \\
& \times \ln (1-\exp \{-t(b \ln (b)-b+1)\})\}
\end{aligned}
$$

Lembremos o fato de que $\ln (1-\alpha) \approx-\alpha$, para $\alpha$ suficientemente pequeno. Então, $\alpha=\exp \{-t(b \ln (b)-b+1)\}$ será pequeno se $b$ for suficientemente grande. Sendo assim, para $b$ suficientemente grande, (3.3.7) será próximo a

$$
\exp \{-2(1+\lambda) \exp \{-t(b \ln (b)-b+1)\}\} \geq c, \quad 0<c<1 .
$$

Por (3.3.4) e (3.3.8),

$$
P\left(W_{t} \leq b t\right) \geq P\left(W_{t} \leq b t \mid N_{t} \leq 2(1+\lambda) e^{\lambda t}\right) P\left(N_{t} \leq 2(1+\lambda) e^{\lambda t}\right) \geq \frac{c}{2}
$$

Mas isto quer dizer que, com probabilidade pelo menos $\frac{c}{2}$, o processo não atinge $b t$, tendo $N_{t}$ partículas contaminadas no instante $t$. Uma vez que o evento $\left\{W_{t} \leq b t\right\}$ equivale a $\{T(0, b t)>t\}$, teremos 


$$
P(T(0, b t)>t) \geq \frac{c}{2}
$$

o que implica em

$$
E(T(0, b t)) \geq \frac{c}{2} t
$$

Logo, para $t$ suficientemente grande,

$$
\frac{E(T(0, b t))}{b t} \geq \frac{c}{2 b}
$$

e portanto, $\gamma>0$.

No que segue procederemos na prova de que $\gamma<1$. Por (i) e (ii) do Lema 3.3.2, $\mathbb{E} T(0, x) \leq \mathbb{E} T(0,1)+\mathbb{E} T(1, x)$ e $\mathbb{E} T(1, x)=\mathbb{E} T(0, x-1)$, e portanto,

$$
\begin{aligned}
\mathbb{E} T(0, x) & \leq \mathbb{E} T(0,1)+\mathbb{E} T(1, x) \\
& =\mathbb{E} T(0,1)+\mathbb{E} T(0, x-1) \\
& \leq \mathbb{E} T(0,1)+\mathbb{E} T(0,1)+\mathbb{E} T(1, x-1) \\
& =2 \mathbb{E} T(0,1)+\mathbb{E} T(0, x-2) \\
& \vdots \\
& =x \mathbb{E} T(0,1)
\end{aligned}
$$

sendo que a última igualdade foi obtida por sucessivas aplicações do Lema 3.3.2. De (3.3.9),

$$
\frac{\mathbb{E} T(0, x)}{x} \leq \mathbb{E} T(0,1)
$$

Mas na prova de (iv) do Lema 3.3 .2 vimos que $\mathbb{E} T(0,1)=\frac{1-e^{-\lambda}}{\lambda}$. Como $\frac{1-e^{-\lambda}}{\lambda}$ é função monótona decrescente em $\lambda$ e $\lim _{\lambda \downarrow 0} \frac{1-e^{-\lambda}}{\lambda}=1$, então $\mathbb{E} T(0,1)<1$. Voltando a (3.3.10), pelo Teorema 3.2.1, 


$$
\gamma=\inf _{x \geq 0} \frac{\mathbb{E} T(0, x)}{x}<1, \quad \text { concluindo que } \quad \gamma<1
$$

\subsection{Obtendo uma cota inferior para a velocidade de contaminação}

Lema 3.4.1. Para $Z_{t}$, fronteira no instante $t, \lim _{t \rightarrow \infty} \frac{Z_{t}}{t}>1$.

Prova: no Lema 3.3.2 temos que quase certamente $\lim _{x \rightarrow \infty} \frac{T(0, x)}{x}=\gamma$. Da definição de limite, para qualquer $\delta>0$, existe $x_{0}$ aleatório, tal que para qualquer $x \geq x_{0}$

$$
(\gamma-\delta) x<T(0, x)<(\gamma+\delta) x
$$

Fixe $\epsilon>0$. Segue que existe $t>0$ tal que $x=\left(\frac{1}{\gamma}+\epsilon\right) t$. Em (3.4.1),

$$
T\left(0,\left(\frac{1}{\gamma}+\epsilon\right) t\right)>(\gamma-\delta)\left(\frac{1}{\gamma}+\epsilon\right) t .
$$

Como $\delta>0$ é arbitrário podemos tomá-lo suficientemente pequeno, de modo que $(\gamma-\delta)\left(\frac{1}{\gamma}+\epsilon\right)>1$, e assim, para $t$ suficientemente grande,

$$
T\left(0,\left(\frac{1}{\gamma}+\epsilon\right) t\right)>t
$$

Mas, o evento $\{T(0, y)>t\}$ implica em $\left\{Z_{t}<y\right\}$, levando-nos a concluir que $Z_{t}<$ $\left(\frac{1}{\gamma}+\epsilon\right) t$. Analogamente, $Z_{t}>\left(\frac{1}{\gamma}-\epsilon\right) t$, e portanto,

$$
\left(\frac{1}{\gamma}-\epsilon\right) t<Z_{t}<\left(\frac{1}{\gamma}+\epsilon\right) t
$$

Como $\epsilon$ é arbitrário, $\lim _{t \rightarrow \infty} \frac{Z_{t}}{t}=\frac{1}{\gamma}>1$, pois pelo Lema 3.3.3, $\gamma<1$. 
Na seqüência nosso objetivo será mostrar que a velocidade do modelo geral, denotada por $v_{g}$, é quase certamente igual a do modelo truncado. Como o modelo geral contém sítios negativos, e conseqüêntemente mais partículas contaminadas do que no modelo truncado, seria natural pensar que $v_{g}$ poderia ser maior. Mas isto não acontece. Uma justificativa empírica é que uma partícula marcada de um sítio negativo tem velocidade 1 (mostramos isto na seção 1.1, através de (1.1.8)), enquanto $v>1$. Logo, é pouco provável que esta partícula marcada alcance $Z_{t}$.

Teorema 3.4.1. Para o modelo geral, com probabilidade positiva, partículas de sítios negativos não alcançam a fronteira entre contaminados e normais.

Prova: para a configuração inicial $\eta$,

$$
\left.P_{\eta} \text { (partículas de sítios negativos não alcançam a fronteira }\right)=\prod_{k<0} P_{\eta}\left(C_{k}\right)
$$

$$
C_{k}:=\text { "partículas do sítio } k \text { não alcançam a fronteira". }
$$

O produtório em (3.4.2) é permitido porque partículas de sítios distintos são independentes.

Lembremos que a configuração inicial é tal que $\eta(k) \sim \operatorname{Pois}(\lambda)$. Mas, 


$$
\begin{aligned}
P(\eta(k) \geq|k|) & =\sum_{j \geq|k|} e^{-\lambda} \frac{\lambda^{j}}{j !} \\
& =e^{-\lambda} \lambda^{|k|} \sum_{j \geq|k|} \frac{\lambda^{j-|k|}}{j(j-1) \cdots(k+1) k !} \\
& \leq e^{-\lambda} \frac{\lambda^{|k|}}{|k| !} \sum_{j \geq|k|} \frac{\lambda^{j-|k|}}{(j-|k|) !} \\
& =e^{-\lambda} \frac{\lambda^{|k|}}{|k| !} \sum_{i \geq 0} \frac{\lambda^{i}}{i !} \\
& =\frac{\lambda^{|k|}}{|k| !},
\end{aligned}
$$

$\mathrm{e}$

$$
\sum_{|k|>0} P(\eta(k) \geq|k|) \leq \sum_{|k| \geq 0} \frac{\lambda^{|k|}}{|k| !}=e^{\lambda}<\infty .
$$

Conseqüentemente, pelo Lema de Borel-Cantelli, $\{\eta(k) \geq|k|\}$ para infinitos $k$ 's tem probabilidade zero. Disso vem que

$$
\begin{gathered}
P_{\eta}\left(C_{k}\right)=\left(P_{\eta}\left(A_{k}\right)\right)^{\eta(k)} \geq\left(P_{\eta}\left(A_{k}\right)\right)^{|k|}, \\
A_{k}:=\text { "uma partícula do sítio } k \text { não alcança a fronteira". }
\end{gathered}
$$

Note que o expoente $|k|$ é permitido porque partículas dentro de um mesmo sítio são independentes.

No Lema 3.4 .1 vimos que $v$ é maior que 1 . Sendo assim, existe $a>1$ tal que $\frac{Z_{t}}{t}>a$, para $t$ suficientemente grande. Mas, para simplificar os cálculos, iremos supor que $\frac{Z_{t}}{t}>a$ para qualquer $t$, pois o caso geral pode ser tratado da mesma maneira. Então, teremos

$$
A_{k}=\{n<|k|+a t, \forall n \geq 1\},
$$


onde $t=\sum_{i=1}^{n} T_{i},\left\{T_{i}\right\}_{i=1}^{n}$ v.a's iid Expon(1). Interpretando (3.4.4), qualquer que seja o número de passos $n$, a partícula que saiu de $k$ não alcança $Z_{t}$. Para que ela pudesse chegar em $Z_{t}$ seriam necessários $|k|+Z_{t}$ passos.

Seja

$$
A_{k}^{n}:=\left\{\frac{n-|k|}{a}<\sum_{i=1}^{n} T_{i}\right\} .
$$

Portanto, $A_{k}=\bigcap_{n \geq 1} A_{k}^{n}$. Calculemos $P_{\eta}\left(\left(A_{k}^{n}\right)^{c}\right)$. Para $s>0$, aplicando a desigualdade de Chebyshev,

$$
\begin{aligned}
P_{\eta}\left(\left(A_{k}^{n}\right)^{c}\right) & =P_{\eta}\left(\sum_{i=1}^{n} T_{i} \leq \frac{n-|k|}{a}\right) \\
& =P_{\eta}\left(\exp \left\{-s \sum_{i=1}^{n} T_{i}\right\} \geq \exp \left\{-s\left(\frac{n-|k|}{a}\right)\right\}\right) \\
& \leq \frac{\left(E\left(\exp \left\{-s T_{1}\right\}\right)\right)^{n}}{\exp \left\{-\frac{s}{a}(n-|k|)\right\}} \\
& =\exp \left\{-\frac{s}{a}|k|\right\}\left(\exp \left\{-\ln (1+s)+\frac{s}{a}\right\}\right)^{n} .
\end{aligned}
$$

Minimizando $-\ln (1+s)+\frac{s}{a}$ em (3.4.5), em relação a $s$, obtemos que $s=a-1>0$. Voltando a (3.4.5),

$$
P_{\eta}\left(\left(A_{k}^{n}\right)^{c}\right) \leq \exp \left\{-\frac{a-1}{a}|k|\right\}\left(\exp \left\{-\ln a+\frac{a-1}{a}\right\}\right)^{n} .
$$

Por (3.4.6),

$$
\begin{aligned}
P_{\eta}\left(A_{k}^{c}\right) \leq \sum_{n=1}^{\infty} P\left(\left(A_{k}^{n}\right)^{c}\right) & =\sum_{n=1}^{\infty} \exp \left\{-\frac{a-1}{a}|k|\right\}\left(\exp \left\{-\ln a+\frac{a-1}{a}\right\}\right)^{n} \\
& =\exp \left\{-\frac{a-1}{a}|k|\right\} \alpha
\end{aligned}
$$


e por conseguinte,

$$
P_{\eta}\left(A_{k}\right) \geq 1-\exp \left\{-\frac{a-1}{a}|k|\right\} \alpha .
$$

Segue, por (3.4.2), (3.4.3) e (3.4.7),

$$
\begin{aligned}
& \left.P_{\eta} \text { (partículas de sítios negativos não alcançam a fronteira }\right) \geq \\
& \prod_{k<0}\left(1-\exp \left\{-\frac{a-1}{a}|k|\right\} \alpha\right)^{|k|} .
\end{aligned}
$$

Agora verificaremos que o produtório em (3.4.8) é positivo. Usaremos o seguinte resultado: para $0<a_{i}<1, \prod_{i=1}^{\infty}\left(1-a_{i}\right)^{i}>0$ se e somente se $\sum_{i=1}^{\infty} i a_{i}<\infty$. De fato, pelo critério da integral,

$$
\sum_{|k| \geq 0} \alpha|k| \exp \left\{-\frac{a-1}{a}|k|\right\}<\infty,
$$

levando-nos a concluir que

$$
P_{\eta} \text { (partículas de sítios negativos não alcançam a fronteira) }>0 \text {, }
$$

e o Teorema 3.4.1 está provado.

Mostremos que $v_{g}=v$. É evidente que $v_{g} \geq v$, uma vez que no modelo geral há um número maior de partículas ativas. Para mostrar que $v \geq v_{g}$ introduziremos o modelo geral tal que partículas acima do sítio $m$ (inteiro positivo) são normais. Note que no Teorema 3.4.1 foi considerado o modelo geral, onde sítios não positivos são contaminados. Para cada $m$ definimos

$$
B_{m}:=\text { "nenhuma partícula de sítios abaixo de } m \text { alcança a fronteira", }
$$


e também denotamos a respectiva velocidade de contaminação por $v_{g}^{(m)}$. No contexto do Teorema 3.4.1 temos $B_{0}$ e $v_{g}^{(0)}=v_{g}$.

Para $m>0$ é imediato que $v_{g}^{(m)} \geq v_{g}^{(0)}$. Por outro lado, pelo Teorema 3.4.1, temos

$$
E\left(I_{\left\{B_{m}\right\}}\right)=E\left(I_{\left\{B_{0}\right\}}\right)=P\left(B_{0}\right)>0 .
$$

Pelo Teorema Estacionário Ergódico

$$
\lim _{m \rightarrow \infty} \frac{1}{m} \sum_{j=1}^{m} I_{\left\{B_{j}\right\}}=P\left(B_{0}\right) \quad \text { quase certamente }
$$

e isto implica que existe $m_{0}$ tal que $B_{m_{0}}$ ocorre. Logo, para este $m_{0}, v_{g}^{\left(m_{0}\right)}=v$. Assim, $v=v_{g}^{\left(m_{0}\right)} \geq v_{g}^{(0)}=v_{g}$, ou seja, $v \geq v_{g}$, levando-nos a concluir que $v=v_{g}$. Acabamos de mostrar que quase certamente a velocidade do modelo geral é igual a do modelo truncado.

\subsection{Conclusões}

Trabalhamos com o modelo truncado, cujos resultados também são válidos para o modelo geral. Através do Teorema Ergódico Subaditivo estabelecemos a existência da velocidade de contaminação. Outro resultado foi uma cota inferior para a velocidade. 


\section{Referências Bibliográficas}

[1] Alves O.S.M., Machado F.P., Popov S. Yu. The Shape Theorem for the Frog Model. The Annals of Applied Probability. Vol. 12, n. 2, p.533-546, 2002.

[2] Alves O.S.M., Machado F.P., Popov S. Yu. The Shape Theorem for the Frog Model with random inicial configuration. Markov Processes Related fields, v.7, p. 525-539, 2002.

[3] Asmussen S.H. Branching Processes. Basel Birkhäuser, 1984.

[4] Bhattacharya R.N., Waymire E.C. Stochastic Processes with Applications. New York, Wiley, 1990.

[5] Csörgö M., Révész P. Strong Approximation in Probability and Statistics. London. Academic Press, 1981.

[6] Derman C. Some contributions to the theory of denumerable Markov Chains. Transac. of American Mathematics Society, 79, p. 541-555, 1955.

[7] Ferrari P.A., Galves J.A. Acoplamento e Processos Estocásticos. In. 21o Colóquio Brasileiro de Matemática. IMPA, Rio de Janeiro, 1997.

[8] Galves A., Nogueira A., Vares M.E. Introdução aos Sistemas Markovianos de Partículas. In. 5o Simpósio Nacional de Probabilidade e Estatística. São Paulo, 1982. 
[9] Gomez C. et al. Engineering and Scientific Computing with Scilab. Boston-Mass. Birkhäuser, 1999.

[10] Grimmett G.R., Stirzaker D.R. Probability and Random Processes. Second edition. Oxford. Oxford Science Publication, 1992.

[11] Hughes B.D. Random Walks and Random Environments. Vol 1. Oxford, Clarendon Press, 1995.

[12] Karatzas I., Shreve S.E. Brownian Motion and Stochastic Calculus. New York. Springer-Verlag, 1988.

[13] Kesten H., Sidoravicius V. The spread of a rumor of infection in a moving population. Preprint: math.PR/0312496 at arXiv.org.

[14] Kesten H., Sidoravicius V. A Shape Theorem for the spread of an infection. Preprint: math.PR/0312511 at arXiv.org.

[15] Kingman J.F.C. Subaditive Ergodic Theory Ann. Probab. 1, p. 883-909, 1973.

[16] Kurkova I., Popov S. Yu., Vachkovskaia M. On infection spreading and competition between independent random walks. Eletronic Journal of Probability. Vol 9, n.11, p.293-315, 2004.

[17] Liggett T.M. An Improved Subadditive Ergodic Theorem. Ann. Prob., 13, p. 1279$1285,1985$.

[18] Liggett T.M. Stochastic Interacting Systems: Contact, Voter and Exclusion Processes. Springer-Verlag, 1999.

[19] Popov S. Frogs and some other interacting random walks models. Discrete Math., Theor. Comput. Sci. AC, p. 277-288, 2003. 
[20] Port S.C. Equilibrium Systems of recurrent Markov Processes. Journal of Math. Anal. Appl. 18, p. 345-354, 1967.

[21] Révész P. Random walks of infinitely many particles. World Scientific Publishing Co., 1994.

[22] Ross S. M. Stochastic Processes. Second edition. New York. Wiley, 1996.

[23] Ross S. M. Introduction to Probability Models. 8nd edition. New York.Academic Press, 1998.

[24] Shiryaev A.N. Probability. Second edition. New York. Springer-Verlag, 1996.

[25] Spitzer F. Principles of Random Walks. Springer-Verlag, Princeton, 1976. 